(C) 2002 International Press

Adv. Theor. Math. Phys. 6 (2002) 703-793

\title{
Supersymmetric Kaluza-Klein
}

\section{reductions of M2 and M5-branes}

\author{
José Figueroa-O'Farrill
}

School of Mathematics, The University of Edinburgh, Scotland, United Kingdom

j.m.figueroa@ed.ac.uk

\section{Joan Simón}

The Weizmann Institute of Physical Sciences, Department of Particle Physics, Rehovot, Israel

jsimon@weizmann.ac.il

\begin{abstract}
We classify and construct all the smooth Kaluza-Klein reductions to ten dimensions of the M2- and M5-brane configurations which preserve some of the supersymmetry. In this way we obtain a wealth of new supersymmetric IIA backgrounds describing composite configurations of D-branes, NS-branes and flux/nullbranes; bound states of D2-branes and strings, D4-branes and NS5-branes, as well as some novel configurations in which the quotient involves nowhere-vanishing transverse rotations to the brane twisted by a timelike or lightlike translation. From these results there also follow novel M-theory backgrounds locally isometric to the M-branes, some of which are time-dependent and all of which are asymptotic to discrete quotients of eleven-dimensional Minkowski spacetime. We emphasise the universality of the formalism by briefly discussing analogous analyses in type IIA/IIB dual to the ones mentioned above. Some comments on the dual gauge theory description of some of our configurations are also included.
\end{abstract}




\section{Introduction and conclusions}

New sectors in string theory have emerged by the embedding of the Melvin universe [1] into string theory $[2,3,4,5,6,7,8,9,10,11,12,13,14,15]$. These are the so-called fluxbranes. Their supergravity description is in terms of the Kaluza-Klein reduction of eleven-dimensional Minkowski spacetime along the orbits of suitable one-parameter subgroups of the Poincaré group.

In our previous work [16] we investigated and classified all smooth KaluzaKlein reductions of eleven-dimensional Minkowski spacetime by one-parameter subgroups of the Poincaré group, paying special attention to those preserving some amount of supersymmetry. These give rise to a wealth of supersymmetric IIA backgrounds including fluxbranes, the then novel nullbranes and combinations thereof. This analysis teaches us that besides fluxbrane sectors, there are new supersymmetric sectors in string theory associated with nullbranes. We will summarise the results of [16] in Section 2.1, but let us comment here briefly on them. The constraint that the IIA background be smooth imposes constraints on the one-parameter subgroup $\Gamma$ by which we reduce. In particular, it follows that $\Gamma$ cannot be compact; in other words, it is not a circle subgroup as in the usual Kaluza-Klein reduction but is diffeomorphic to the real line. Since Kaluza-Klein reduction is usually phrased in terms of circle subgroups, one can still do that here provided one performs the reduction by $\Gamma$ in two steps.

It will be convenient to generalise the discussion and consider not just the reduction of Minkowski spacetime but that of an arbitrary M-theory background $\mathcal{M}=\left(M, g, F_{4}\right)$ by a noncompact one-parameter Lie subgroup $\Gamma$ of the symmetry group of $\mathcal{M}$. The first step consists in performing a discrete quotient to obtain another M-theory background. To this effect one first chooses a co-compact discrete subgroup $\Gamma_{0} \subset \Gamma$ (that is, a subgroup such that the quotient group $\Gamma / \Gamma_{0}$ is compact, e.g., if $\Gamma \cong \mathbb{R}$ then $\Gamma_{0} \cong \mathbb{Z}$ ) and performs the quotient by $\Gamma_{0}$. The resulting background $\mathcal{M} / \Gamma_{0}$ is a smooth supersymmetric M-theory background which is locally isometric to $\mathcal{M}$ : it consists of making identifications along the orbits of the Killing vector generating $\Gamma$ at equal intervals in the parameter space. The choice of this interval implies a choice of scale relative to which the (possibly varying) size of the M-theory circle is measured.

Having quotiented by $\Gamma_{0}$, it is the circle subgroup $\Gamma / \Gamma_{0}$ which acts nontrivially on $\mathcal{M} / \Gamma_{0}$. Its orbits are the $\mathrm{M}$-theory circles, which may be of varying size. The second step in the reduction is then the standard KaluzaKlein reduction of $\mathcal{M} / \Gamma_{0}$ by the circle subgroup $\Gamma / \Gamma_{0}$. 
The advantage of performing the reduction along $\Gamma$ in two steps, apart from placing the dimensional reduction squarely in the familiar context of circle reductions, is that the result of the first step is often interesting in its own right.

In fact, in [16] we discovered a novel reduction of eleven-dimensional Minkowski spacetime: a generalised fluxbrane in type IIA which we dubbed a nullbrane, since the RR 2-form field strength is null and moreover the corresponding Killing vector $\xi$ has a component which is a null rotation. The subgroup $\Gamma$ generated by this Killing vector is noncompact, parametrised by a real number $t$. The isomorphism $\mathbb{R} \rightarrow \Gamma$ is given explicitly by $t \mapsto g(t):=$ $\exp t \xi$. This map is a group homomorphism: $g(t) g\left(t^{\prime}\right)=g\left(t+t^{\prime}\right)$, whence if we choose a scale $R$, the elements $\Gamma_{0}=\{g(n R) \mid n \in \mathbb{Z}\}$, form a co-compact discrete subgroup isomorphic to $\mathbb{Z}$. The quotient $\mathbb{R}^{1,10} / \Gamma_{0}$ of Minkowski spacetime by $\Gamma_{0}$ is a smooth supersymmetric M-theory background, locally isometric to Minkowski spacetime. It is moreover time-dependent, since the null rotation involves time nontrivially. It has been dubbed the "nullbrane" in [17] because the circle reduction to IIA along the orbits of $\Gamma / \Gamma_{0}$ gives rise to the nullbrane of [16]. We will call it here the "eleven-dimensional nullbrane" to avoid confusion.

Similarly, there are "eleven-dimensional fluxbranes" which are obtained by performing a discrete quotient of $\mathbb{R}^{1,10}$ by $\Gamma_{0} \subset \Gamma$, where $\Gamma$ is now such that $\mathbb{R}^{1,10} / \Gamma$ is a IIA fluxbrane.

The above discussion holds in any number $D+1$ of dimensions-in particular in ten dimensions. Ten-dimensional fluxbranes, that is, $\mathbb{R}^{1,9} / \Gamma_{0}$, allow a conformal field theory (CFT) $[18,19,20,21,22,23]$ description in terms of resolutions of the ordinary euclidean orbifolds. Analogously, ten-dimensional nullbranes resolve null (or parabolic) orbifolds [24, 25]. The geometry of these null orbifolds has been recently discussed in [26, 27]. It was further noticed in [27] that, by going to the light cone gauge such spacetimes allow an interpretation in terms of a Big Crunch phase connected to a Big Bang phase through a point where our spacetime manifold is non-Hausdorff. The nullbrane not only resolves the fixed points of the null orbifold and its nonHausdorff nature, but it is also free of closed causal curves and it is stable against the formation of black holes when probed by particles at least at low energies and for a large enough number of spacetime dimensions [28, 29]. The geometry of the eleven-dimensional nullbrane is a metric product of a flat seven-dimensional euclidean space (which can be further compactified) and a four-dimensional locally minkowskian spacetime which can be viewed as the total space of a circle fibration over a 3-dimensional manifold, where the radius of the circle varies with time and reaches a minimum nonzero size 
$R$, which is nothing but the scale in the previous discussion. These properties seem to allow the use of string perturbation theory in these discrete quotients $[17,29]$, extending the original formulation for the null orbifold given in [27].

The existence of these backgrounds with cosmological interpretations motivates and suggests a natural mathematical problem, namely the classification of smooth supersymmetric M-theory or type IIA/IIB backgrounds which are obtained by discrete quotients (often termed orbifolds even if the resulting quotient is smooth) of a given one. This is a much harder problem already for the case of Minkowski spacetime and it definitely does not follow from the results of [16]. Its solution would require classifying all the discrete subgroups $\Gamma_{0}$ of the corresponding Poincaré group which act freely and properly discontinuously on Minkowski spacetime and which preserve some supersymmetry. As discussed in $[30,31]$ supersymmetry in eleven dimensions requires that $\Gamma_{0}$ be contained in the subgroup $\operatorname{Spin}(7) \ltimes \mathbb{R}^{9}$ of the Poincaré group. The classification of smooth flat supersymmetric M-theory backgrounds would include, in particular, the classification of crystallographic subgroups of $\operatorname{Spin}(7) \subset \mathrm{SO}(8)$ : a hard problem in practice despite the existence of a powerful algorithm. Some comments and partial results concerning smooth time-dependent backgrounds constructed in this way can be found in Section 7 of [17].

In this paper, as in [16], we will not emphasise the discrete quotients of the M-theory backgrounds, but it is worth remarking that from our results there follows a classification of those discrete quotients by (infinite) cyclic subgroups. As some of the subgroups we will exhibit act nontrivially in the time direction, some of the M-theory backgrounds resulting from the corresponding discrete identifications will be time-dependent. There has been much recent interest in this class of backgrounds, as evidenced by the recent work on spacelike branes [32, 33, 34, 35], Sen's proposal [36, 37] that dynamical rolling of the tachyon of open string field theory can lead to interesting cosmologies, double Wick rotations of stationary configurations $[38,39,40,41,42]$, coset models [43, 44] and related orbifold constructions $[45,46,47,48,49]$. The time-dependent backgrounds implicit in our results hence add to the existing classes of examples of such backgrounds. It should be remarked that unlike the ones found in [16], these will not be flat, but they will be asymptotic to $\mathbb{R}^{1,10} / \Gamma_{0}$, as corresponds to excitations of these new M-theory vacua. We will call them asymptotically locally flat spaces.

The purpose of the present work is twofold. Firstly, it is natural to ask about which D-branes, NS-branes and other dynamical objects in string theory exist in flux/nullbrane sectors. This question has been partially ad- 
dressed for the fluxbrane sector using CFT techniques [50, 51]. In the following, we shall adopt a low-energy effective closed string description to fully answer this point. In this way, we shall not only classify all the allowed supersymmetric configurations, but we shall find their explicit supergravity realisation at the same time. If one is interested in classifying composite configurations of branes, waves, monopoles,... and flux/nullbranes, it is natural to consider the same configuration at strong coupling, where they should be described in terms of the uplifted known M-theory backgrounds satisfying certain nontrivial global identifications reminiscent of the twisted identifications associated with flux/nullbranes in flat spacetime. Thus, our classification problem of composite configurations in type IIA is equivalent to a classification of certain one-parameter subgroups $\Gamma$ of the symmetry group of an M-brane background $\mathcal{M}$. The Kaluza-Klein reduction $\mathcal{M} / \Gamma$ will then give rise to the desired composite configurations. This has already been shown for a particular configuration in [52] through a probe analysis of D0-branes in flux 5-brane (F5-brane) backgrounds. Similar ideas were also pointed out in the appendix of [23]. On the other hand, performing the reduction in steps: first quotienting by a discrete subgroup $\Gamma_{0} \subset \Gamma$ and then quotienting the resulting $M$-theory background by the circle subgroup $\Gamma / \Gamma_{0}$, will give rise, after the first step, to new eleven-dimensional backgrounds $\mathcal{M} / \Gamma_{0}$, some of which will be time-dependent and all of which will be asymptotic to $\mathbb{R}^{1,10} / \Gamma_{0}$.

The second goal of this work is to make progress in the classification of supersymmetric M-theory and type IIA supergravity backgrounds. Due to the equivalence stated above, it is natural to make a thorough analysis of all smooth Kaluza-Klein reductions of a given fixed M-theory background $\mathcal{M}$. It will turn out that for curved spacetimes, such as the ones describing M-branes, new reductions arise which were not taken into account in the past and are not possible in Minkowski spacetime.

For example, it is possible to reduce along the orbits generated by Killing vectors involving time translations and rotations transverse to the brane, but which nevertheless are everywhere spacelike, so as to avoid the existence of manifest closed timelike curves. ${ }^{1}$ These novel reductions appear whenever the unit sphere transverse to the brane is odd-dimensional, because such spheres will admit nowhere-vanishing Killing vectors. This is the case for M2-branes or delocalised M5-branes, but it is certainly a much more general phenomenon, present in the delocalised $\mathrm{M}$-wave [53] and in some intersecting

\footnotetext{
${ }^{1}$ Although such a restriction is perfectly legitimate from a physical point of view, one cannot discard the possibility of obtaining novel string backgrounds by reducing along the orbits of a freely-acting Killing vector $\xi$ after excising from the spacetime those regions where $\xi$ fails to be spacelike, with the usual completeness caveats.
} 
brane backgrounds [54], for instance.

A second example of novel reductions results whenever the brane is delocalised. In such cases one can consider Killing vectors involving linear combinations of a translation tangent to the worldvolume of the brane and a translation transverse to it. If both translations are spacelike, the type IIA configuration describes non-threshold bound states. If one of them is lightlike, the IIA observer no longer measures an asymptotically Minkowski spacetime, but a wave propagating at the speed of light. Whenever one of the translations is timelike, the physical interpretation remains unclear to us.

It is a natural question to ask about the global causal structure of the spacetimes obtained by reducing along Killing vectors which act nontrivially on time. This question remains open for the former set of novel reductions discussed above, but we will show that for the latter set there are no closed causal curves whenever the Killing vector is everywhere spacelike, even if it contains timelike and lightlike translations.

It should be clear that any of the techniques mentioned above also applies to any on-shell background in type IIA/IIB supergravity. In this context, our analysis must be understood as the classification of all inequivalent quotients (discrete or not) of such a given background by a one-parameter subgroup of symmetries. As will be discussed in the last section of this paper, both constructions, the one obtained from M-theory through Kaluza-Klein reduction and the one directly constructed in type IIA are equivalent through a TST chain of dualities, $\mathrm{T}$ standing for T-duality and S for S-duality transformations, respectively.

In the context of the AdS/CFT correspondence [55], some of our configurations (composites of D-branes and fluxbranes), when studied in certain decoupling limits, provide us with supergravity duals of the dipole theories introduced in $[56,57,58,59,60]$. Indeed, our configurations depend on some set of parameters (related to the charge of the fluxbranes) which can be held fixed in a Maldacena-type limit [61,62]. They can thus be viewed as some sort of deformation parameters of the corresponding near horizon geometries of D-branes. Our analysis shows the existence of further supersymmetric configurations both in the flux- and nullbrane sectors. Indeed, the identifications underlying dipole theories involve the R-symmetry group transverse to the brane. We point out the possibility of making identifications involving transverse and longitudinal (either spacelike or lightlike) directions to the brane at the same time. Their dual gauge theory description involves supersymmetric Yang-Mills with the adjoint matrices satisfying twisted con- 
ditions generalising the ones written before $[56,63]$. Similar techniques were used also in [64].

We would like to emphasise the universality of the construction presented in this paper. Even though we shall restrict ourselves to M2 and M5-brane configurations in this work, it is clear that any supersymmetric configuration, with enough symmetry, of a $D+1$ supergravity theory (ordinary or gauged) for which a consistent Kaluza-Klein truncation to a $D$-dimensional supergravity theory is known, would generate new $D$-dimensional configurations by using the same methods developed below. In particular, extensions to M-waves and MKK-monopoles [53], intersections of M-branes [54], supersymmetric wrapped branes, antibranes, multicentred configurations or supergravity duals of non-commutative gauge theories, among others, are conceptually straightforward. It is particularly interesting to address these questions in supersymmetric backgrounds of the form $\operatorname{AdS}_{p} \times S^{q}$ both in Mtheory and in type IIB. The BTZ black hole [65] construction out of $\mathrm{AdS}_{3}$ [66] and the dual description of string theory in these backgrounds in terms of supersymmetric Yang-Mills theories opens many interesting questions both in the gravity and gauge theory sides. This is currently under investigation and some results will appear elsewhere $[67,68]$.

In what remains of this introductory section, we provide a self-contained and hopefully comprehensive description of the geometrical set-up underlying Kaluza-Klein reductions. It also fixes the notation and conventions used in the rest of the paper. Section 1.2 deals with an arbitrary bosonic background having certain group of isometries, whereas in Section 1.3 we explain which is the criterion for preservation of supersymmetry. In this way, we are naturally led to introduce the notion of moduli space of supersymmetric Kaluza-Klein reductions. In Section 1.4, we present the argument that will allow us to determine this moduli for the M2- and M5-brane configurations in the body of the paper. Finally, Section 1.5 details the organisation of the rest of the paper and a brief summary of its results.

\subsection{Supersymmetric M-theory backgrounds}

The bosonic fields of eleven-dimensional supergravity $[69,70]$ are a lorentzian metric $g$ and a closed four-form $F_{4}$ defined on an eleven-dimensional spin manifold $M$. The bosonic equations of motion are a generalisation of the Einstein-Maxwell equations in four and five dimensions. They consist of a nonlinear Maxwell-type equation for $F_{4}$ :

$$
d \star F_{4}=\frac{1}{2} F_{4} \wedge F_{4}
$$


and an Einstein-type equation for $g$ whose explicit form need not concern us here, but which relates the Einstein tensor of $g$ to the energy momentum tensor of $F_{4}$-an algebraic tensor depending on $g$ and quadratically on $F_{4}$.

By hypothesis $M$ has a spin structure and hence a bundle of spinors, which is a (symplectic) real bundle of rank 32. The supersymmetry of the supergravity action (with fermions included) induces a covariant derivative operator $\mathcal{D}$ on spinors. It is defined as the supersymmetry variation of the gravitino $\Psi_{M}$ restricted to the subspace of the configuration space where the gravitino vanishes. In other words, if $\varepsilon$ is a spinor, then

$$
\mathcal{D}_{M} \varepsilon=\left.\delta_{\varepsilon} \Psi_{M}\right|_{\Psi=0} .
$$

We will not need the explicit expression of $\mathcal{D}$; suffice it to say that it can be written in the form $\mathcal{D}_{M}=\nabla_{M}+\Omega_{M}$, where $\nabla$ is the spin connection and $\Omega_{M}$ is an endomorphism of the spinor bundle depending algebraically in $g$ and linearly in $F_{4}$. A nonzero spinor $\varepsilon$ which obeys $\mathcal{D}_{M} \varepsilon=0$ is called a Killing spinor, as they are the "square roots" of Killing vectors. A pair $\left(g, F_{4}\right)$ satisfying the field equations (with fermions set to zero) is called a (bosonic) background. A background is supersymmetric if it possesses Killing spinors. Since the Killing spinor equation is linear, the space of Killing spinors is a vector space, and since the equation is first order, its dimension is not bigger than the rank of the spinor bundle, here 32 . A background $\left(g, F_{4}\right)$ is said to preserve a fraction $\nu$ of the supersymmetry if the dimension of the space of Killing spinors is $32 \nu$. The space of backgrounds is stratified by the possible values $\left\{0, \frac{1}{32}, \frac{1}{16}, \ldots, 1\right\}$ of $\nu$. At the time of writing it is not known whether all strata are nonempty.

\subsection{The geometry of Kaluza-Klein reductions}

Ten-dimensional type IIA supergravity can be defined as the Kaluza-Klein reduction of eleven-dimensional supergravity along a spacelike direction [71, 72, 73]. This means that if the Kaluza-Klein reduction of an M-theory background exists, it is automatically a type IIA background and moreover every IIA background arises in this way (at least locally). Of course, not every M-theory background can be reduced, as this requires the existence of a symmetry.

By an (infinitesimal) symmetry of a background $\left(g, F_{4}\right)$ we mean a vector field $\xi$ on $M$ such that

- $\xi$ is Killing: $\mathcal{L}_{\xi} g=0$; and 
- $\xi$ preserves $F_{4}: \mathcal{L}_{\xi} F_{4}=0$-equivalently $d i_{\xi} F_{4}=0$, since $F_{4}$ is closed.

Now suppose that an infinitesimal symmetry $\xi$ integrates to an action of a one-dimensional group $\Gamma$ (either $\mathbb{R}$ or $S^{1}$ ) obeying the following two properties:

1. the action is free; and

2. the norm of $\xi$ never vanishes.

The first property involves two separate conditions: one infinitesimal, that the vector field $\xi$ never vanishes; and one of a more global nature, that every point in $M$ should have trivial stabiliser. This guarantees that the space $N=M / \Gamma$ of $\Gamma$-orbits is a smooth manifold. The second property means that $N$ inherits a metric: lorentzian if $\xi$ is spacelike or riemannian if it is timelike. As in [16], we will concentrate solely on the case of a spacelike $\xi$.

The condition that $\xi$ does not vanish is a necessary condition for the smoothness of the quotient. In many of the examples we will consider in this paper, $\xi$ will tend to zero as we approach the horizon. Such a zero need not have geometric meaning, as the coordinate system in which our ansätze are written are singular in the horizon. One way to determine whether or not this zero of the vector field yields a singularity in the quotient would be to extend the solution beyond the horizon, as the brane ansätze we will write down only cover the spacetime exterior to the brane. In order to keep this paper down to a reasonable length, we will choose to ignore these potential singularities and take the conservative approach that our solutions only describe the spacetime exterior to the brane, even though the physics of these potential singularities in the throats of the different branes and their resolutions, if any, are very interesting questions for future research.

Since it plays an important role in our approach, we now describe in some detail the geometric underpinning of the Kaluza-Klein ansatz. We think of the original spacetime $M$ as the total space of a principal $\Gamma$-bundle $\pi: M \rightarrow N=M / \Gamma$, where $\pi$ is the map taking a point in $M$ to the $\Gamma$ orbit on which it lies. At every point $p$ in $M$, the tangent space $T_{p} M$ of $M$ at $p$ decomposes into two orthogonal subspaces: $T_{p} M=\mathcal{V}_{p} \oplus \mathcal{H}_{p}$, where the vertical subspace $\nu_{p}=\operatorname{ker} \pi_{*}$ consists of those vectors tangent to the $\Gamma$-orbit through $p$, and the horizontal subspace $\mathcal{H}_{p}=\mathcal{V}_{p}^{\perp}$ is its orthogonal complement relative to the metric $g$. The resulting decomposition is indeed a direct sum by virtue of the nowhere-vanishing of the norm of $\xi$, whose value at $p$ spans $\mathcal{V}_{p}$ for all $p$. The derivative map $\pi_{*}$ sets up an isomorphism between $T_{p} M$ and $T_{q} N$, where $\pi(p)=q$. There is a unique metric on $N$ for 
which this isomorphism is also an isometry and it is defined as follows. We choose a point $p$ in the fibre above $q$. Then if $X, Y \in T_{q} N$, we define their inner product ${ }^{2} h^{\prime}(X, Y)=g(\tilde{X}, \tilde{Y})$, where $\tilde{X}, \tilde{Y} \in \mathcal{H}_{p}$ obeying $\pi_{*} \tilde{X}=X$ and $\pi_{*} \tilde{Y}=Y$ are the horizontal lifts of $X$ and $Y$, respectively. This does not actually depend on the choice of $p$ because $\Gamma$ acts by isometries.

The horizontal sub-bundle $\mathcal{H}$ gives rise to a connection one-form $\alpha$ on $M$ such that $\mathcal{H}=\operatorname{ker} \alpha$ and such that $\alpha(\xi)=1$, where $\xi$ is the Killing vector generating the $\Gamma$-action. Relative to a local trivialisation, and letting $z$ be a coordinate along the $\Gamma$-orbits, we can write $\alpha=d z+A$, where $A$ is a locallydefined one-form on $N$. In terms of this data (and omitting pullbacks by $\pi$ ) the metric $g$ on $M$ can be written as

$$
g=h^{\prime}+e^{4 \Phi / 3}(d z+A)^{2},
$$

where $e^{2 \Phi / 3}$ is the norm of the Killing vector $\xi$, which in this trivialisation is given by $\xi=\partial_{z}$. The function $\Phi: N \rightarrow \mathbb{R}$ is the dilaton. To make contact with the metric which appears in the effective action of the type IIA string, it is convenient to conformally rescale the metric on $N$ by a function of the dilaton. Doing this we finally arrive at the familiar string-frame Kaluza-Klein ansatz for the dimensional reduction of eleven-dimensional supergravity to type IIA supergravity:

$$
g=e^{-2 \Phi / 3} h+e^{4 \Phi / 3}(d z+A)^{2} .
$$

The four-form $F_{4}$ also reduces and gives rise to two forms on $N$ : the NSNS three-form $\mathrm{H}_{3}$ and the RR four-form $H_{4}$. To see how this comes about, let us first decompose $F_{4}$ as follows

$$
F_{4}=G_{4}-\alpha \wedge G_{3},
$$

where $\alpha$ is the connection one-form defined above. The curvature two-form $d \alpha$ is both horizontal, so that $\imath_{\xi} d \alpha=0$ and invariant, so that $\mathcal{L}_{\xi} d \alpha=0$. (In this case, invariance follows from horizontality since $d \alpha$ is closed.) To prove horizontality, we first observe that $\alpha=\xi^{b} /\|\xi\|^{2}$, where $\xi^{b}$ is the one-form defined by $\xi^{b}(X)=g(\xi, X)$ for all vectors field $X$ on $M$, is invariant ${ }^{3}$. This means that $\imath_{\xi} d \alpha+d l_{\xi} \alpha=0$, but since $\alpha(\xi)=1$ and hence constant, this is precisely horizontality of $d \alpha$.

Forms which are both horizontal and invariant are called basic, since they are pull-backs of forms on the base $N$. The curvature two-form $d \alpha$ is the pull-back of the RR two-form field-strength $H_{2}=d A$. Let us remark

\footnotetext{
${ }^{2}$ The reason for the prime in the notation will become obvious below.

${ }^{3}$ This is equivalent to the physical statement that the photon carries no charge.
} 
in passing that in coordinates adapted to $\xi$, so that $\xi=\partial_{z}$, a form is basic if and only if it has no $d z$ component (horizontality) and does not depend explicitly on $z$ (invariance).

We now claim that both $G_{4}$ and $G_{3}$ are basic. It is clear from the above expression that $G_{3}=-\imath_{\xi} F_{4}$, so that it is manifestly horizontal. Invariance of $F_{4}$ means that $G_{3}$ is closed, whence it is basic. It is therefore the pull-back of a three-form on $N$ : the NSNS three-form field-strength $H_{3}=d B_{2}$. Finally, we observe that $G_{4}$ is also basic. It is manifestly horizontal, and invariance follows by a simple calculation

$$
\begin{aligned}
\mathcal{L}_{\xi} G_{4} & =d \imath_{\xi} G_{4}+\imath_{\xi} d G_{4} \\
& =\imath_{\xi}\left(-d \alpha \wedge G_{3}\right) \\
& =0
\end{aligned}
$$

where we have used that $G_{3}, G_{4}$ and $d \alpha$ are horizontal. This means that $G_{4}$ is the pull-back of a four-form on $N$. It is convenient to write that four-form as $H_{4}-H_{3} \wedge A$, where $H_{4}$ is the $\mathrm{RR}$ four-form field-strength.

In the local trivialisation used above, we can rewrite the above decomposition of $F_{4}$ in a more familiar form (omitting pullbacks)

$$
F_{4}=H_{4}-d z \wedge H_{3} .
$$

In summary, if $\left(M, g, F_{4}\right)$ is an M-theory background admitting a free $\Gamma$ action $\left(\Gamma=\mathbb{R}\right.$ or $\left.\Gamma=S^{1}\right)$ with spacelike orbits, then $\left(N=M / \Gamma, h, \Phi, H_{2}=\right.$ $\left.d A, H_{3}, H_{4}\right)$ is a type IIA background. Conversely any IIA background $\left(N, h, \Phi, H_{2}, H_{3}, H_{4}\right)$ can be lifted locally to an M-theory background $\left(M, g, F_{4}\right)$ possessing a spacelike symmetry in such a way that the Kaluza-Klein reduction of $\left(M, g, F_{4}\right)$ along that symmetry reproduces the IIA background we started out with.

Some of the backgrounds we will be studying admit a simpler description in terms of the dual seven-form $F_{7}=\star F_{4}$. The Kaluza-Klein reduction of $F_{7}$ is very similar to that of $F_{4}$. We start by decomposing $F_{7}$ as

$$
F_{7}=G_{7}+\alpha \wedge G_{6},
$$

where $G_{7}$ and $G_{6}$ are horizontal. This means that $G_{6}=\imath_{\xi} F_{7}$. We claim that $G_{6}$ and $G_{7}$ are also invariant, whence basic. To see this we compute their Lie derivative with respect to $\xi$ :

$$
L_{\xi} G_{6}=L_{\xi} \imath_{\xi} F_{7}=\imath_{\xi} L_{\xi} F_{7}=0,
$$


since $F_{7}$ is invariant. Similarly, using that $G_{7}=F_{7}-\alpha \wedge G_{6}$, we calculate

$$
L_{\xi} G_{7}=L_{\xi}\left(F_{7}-\alpha \wedge G_{6}\right)=-L_{\xi} \alpha \wedge G_{6}=0,
$$

where we have used that $\alpha$ is invariant. In summary, since they are basic, $G_{6}$ and $G_{7}$ are pullbacks of forms on the base. In adapted coordinates where $\xi=\partial_{z}$ and $\alpha=d z+A$, we can write (omitting pullbacks)

$$
F_{7}^{*}=H_{7}+d z \wedge H_{6},
$$

where $H_{6}$ pulls back to $G_{6}$ and $H_{7}$ pulls back to $G_{7}-A \wedge G_{6}$.

It is possible to trace the Hodge-dual across the reduction and to relate $H_{7}$ and $H_{6}$ to $H_{4}$ and $H_{3}$ and the fields to which the eleven-dimensional metric reduces. One finds

$$
\begin{aligned}
& H_{6}=\star_{10}\left(H_{4}+A \wedge H_{3}\right) \\
& H_{7}=A \wedge \star_{10} H_{4}+\star_{10}{ }^{\sharp}\left(A \wedge H_{3}\right)-e^{-2 \Phi} \star_{10} H_{3},
\end{aligned}
$$

where $\star_{10}$ is the ten-dimensional Hodge star operator relative to the metric $h$ and $A^{\sharp}$ is the vector field dual to the RR one-form $A$.

One can also invert the above relation and express $H_{3}$ and $H_{4}$ in terms of $H_{6}$ and $H_{7}$. With the same notation as above, the result is the following

$$
\begin{aligned}
& H_{3}=e^{2 \Phi} \star_{10}\left(H_{7}-A \wedge H_{6}\right) \\
& H_{4}=\star_{10} H_{6}-e^{2 \Phi} A \wedge \star_{10} H_{7}+e^{2 \Phi} \star_{10} \imath_{A^{\sharp}}\left(A \wedge H_{6}\right),
\end{aligned}
$$

which we record here for future reference.

\subsection{Supersymmetric Kaluza-Klein reductions}

Closely tied to the symmetries of a supergravity background are its supersymmetries, which manifest themselves through Killing spinors. In this section we review what happens to supersymmetry under Kaluza-Klein reduction.

Every Killing vector $\xi$ acts naturally on a spinor $\varepsilon$ via the spinorial Lie derivative introduced by Lichnerowicz (see, e.g. [74] and more recently [75] for applications closer to the present context) and defined by

$$
\mathcal{L}_{\xi} \varepsilon=\nabla_{\xi} \varepsilon+\frac{1}{4} d \xi^{b} \cdot \varepsilon,
$$

where $\nabla$ is the spin connection and where - means the Clifford action of forms on spinors. Choosing a local frame, we can write

$$
\mathcal{L}_{\xi} \varepsilon=\nabla_{\xi} \varepsilon+\frac{1}{4} \nabla_{a} \xi_{b} \Gamma^{a b} \varepsilon .
$$


The spinorial Lie derivative enjoys many of the properties that we expect of a Lie derivative; in particular, it is a derivation

$$
\mathcal{L}_{\xi}(f \varepsilon)=(\xi f) \varepsilon+f \mathcal{L}_{\xi} \varepsilon,
$$

for any function $f$. Furthermore one can show that for any vector field $X$,

$$
\left[\mathcal{L}_{\xi}, \nabla_{X}\right]=\nabla_{[\xi, X]}
$$

and if in addition $\xi$ preserves $F_{4}$, then

$$
\left[\mathcal{L}_{\xi}, \mathcal{D}_{X}\right]=\mathcal{D}_{[\xi, X]} .
$$

This implies that the Lie derivative along $\xi$ of a Killing spinor is again a Killing spinor, and hence the space of Killing spinors becomes a linear representation of the group $\Gamma$ generated by $\xi$.

It follows from the definition of type IIA supergravity as the KaluzaKlein reduction of eleven-dimensional supergravity, that the supersymmetries of the IIA background are precisely the $\Gamma$-invariant Killing spinors of the M-theory background. Notice that the condition $\mathcal{L}_{\xi} \varepsilon=0$ for $\varepsilon$ a Killing spinor is actually an algebraic condition, since $\mathcal{L}_{\xi}-\mathcal{D}_{\xi}$ is a zeroth order operator. This is nothing but the condition which arises from the supersymmetry variation of the IIA dilatino fields. Thus if an M-theory background admits a Kaluza-Klein reduction along the orbits of a group $\Gamma$ such that there are $\Gamma$-invariant Killing spinors, the resulting IIA background will be supersymmetric. We will call this a supersymmetric Kaluza-Klein reduction. The IIA background will preserve a fraction $\nu$ of the supersymmetry, where $32 \nu$ is the dimension of the space of $\Gamma$-invariant Killing spinors of the corresponding M-theory background.

Given a supersymmetric M-theory background $\left(M, g, F_{4}\right)$ with symmetries, the problem of finding supersymmetric Kaluza-Klein reductions can be phrased in the following terms. Let $G$ be the Lie group of symmetries of the background and let $\mathfrak{g}$ denote its Lie algebra, consisting of infinitesimal symmetries, namely those Killing vectors on $M$ which also preserve $F_{4}$. Let $\mathcal{T}$ be the subset of $\mathfrak{g}$ corresponding to those spacelike Killing vectors which integrate to a free group action preserving some Killing spinors. The subset $\mathcal{T}$ parametrises the supersymmetric Kaluza-Klein reductions of the background $\left(M, g, F_{4}\right)$; however not all points in $\mathcal{T}$ correspond to physically distinct reductions. Indeed, the action of $G$ on $M$ induces the adjoint action on $\mathfrak{g}$, and this action preserves the subset $\mathcal{T}$. Two points in $\mathcal{T}$ which are $G$-related, correspond to two physically indistinguishable supersymmetric reductions, as they are related by a change of variables corresponding to a symmetry. The space $\mathcal{T}$ is also preserved by rescaling the Killing vectors. 
Indeed, notice that if $\xi \in \mathcal{T}$, then $R \xi \in \mathcal{T}$ for every nonzero real number $R \in \mathbb{R}^{\times}$. Although the scale $R$ has physical meaning-for example, it is related to the radius of the M-theory circle in the standard Kaluza-Klein reduction-it is natural, from a mathematical perspective at least, to identify collinear elements in $\mathcal{T}$ and define the moduli space $\mathcal{M}$ of supersymmetric Kaluza-Klein reductions as the set of orbits of the action of $G \times \mathbb{R}^{\times}$on $\mathcal{T}$; in other words, as the set of equivalence classes $[X]$ where $X \in \mathcal{T}$, where $X \sim R g X g^{-1}$ for all $g \in G$ and $R \in \mathbb{R}^{\times}$. The number of Killing spinors left invariant by $X$ and by $R g X g^{-1}$ is clearly the same, whence the space $\mathcal{M}$ is stratified by the value of $\nu$, the fraction of the supersymmetry preserved by the IIA background, which will be a further fraction of that preserved by the M-theory background from which we reduce. In [16] we determined $\mathcal{M}$ for the flat eleven-dimensional $\mathrm{M}$-theory vacuum and in this paper we determine $\mathcal{M}$ for the M2 and M5-brane solutions by mapping the problem essentially (but not quite) to the flat case, albeit with a restricted symmetry group. A similar method also allows us to determine $\mathcal{M}$ for the purely gravitational M-wave and Kaluza-Klein monopole [53].

We should remark that the failure of the problem to map precisely to the flat case, far from being a problem, is actually responsible for the existence of novel reductions by vector fields which would have Killing horizons in flat space but which are spacelike relative to the brane metric.

\subsection{Classifying supersymmetric brane reductions}

The classification problem of supersymmetric reductions has two parts. One is the classification proper: determining the moduli space $\mathcal{N}$ of the given background. Then given $\mathcal{M}$, a second part of the problem is to perform the reduction explicitly. Although one can address this problem for an arbitrary supersymmetric background, in practice only the most symmetric backgrounds admit an explicit solution.

Since Killing spinors square to Killing vectors, one way to generate backgrounds with sufficient symmetry is to look for backgrounds preserving a large fraction of the supersymmetry. In [16] we considered the case of flat space. Due to the high degree of symmetry present in the flat background, we were able to employ group-theoretical methods to solve the first part of the problem and classify all the supersymmetric (generalised) fluxbranes. We were then able to write down explicit formulae for the resulting IIA backgrounds.

In the present paper we extend these results to the M2 and M5-brane 
half-BPS backgrounds. These brane-like solutions we consider in this paper are asymptotically flat and crucial to our approach is that they inherit isometries and supersymmetries from the asymptotic geometry. Indeed, both Killing vectors and Killing spinors are determined uniquely by their asymptotic values. Moreover, as the following simple argument will show, this correspondence is equivariant with respect to the action of the Killing vectors on the Killing spinors, reducing the problem in essence to the flat space case, albeit with a restricted Poincaré group.

Consider for simplicity a typical electric $p$-brane solution in $d$ dimensions:

$$
\begin{aligned}
g & =e^{2 A} d s^{2}\left(\mathbb{E}^{1, p}\right)+e^{2 B} d s^{2}\left(\mathbb{E}^{d-p-1}\right) \\
F_{4} & =\operatorname{dvol}\left(\mathbb{E}^{1, p}\right) \wedge d C,
\end{aligned}
$$

where $A, B, C$ are functions of the radial distance $r$ in the transverse space $\mathbb{E}^{d-p-1}$ approaching 0 as $r \rightarrow \infty$. (In the magnetic ansatz we would replace $F_{4}$ with $\star F_{4}$.) The asymptotic geometry is therefore flat and invariant under $\operatorname{ISO}(1, d-1)$, whereas the brane solution is only invariant under a subgroup $G=\operatorname{ISO}(1, p) \times \mathrm{SO}(d-p-1)$. The Killing spinors for such a background are of the form

$$
\varepsilon=e^{D} \varepsilon_{\infty},
$$

where $D$ is another function of the transverse radius approaching 0 at infinity and where the asymptotic value $\varepsilon_{\infty}$ is a (covariantly) constant spinor in flat space subject to a condition of the form

$$
\operatorname{dvol}\left(\mathbb{E}^{1, p}\right) \cdot \varepsilon_{\infty}=\varepsilon_{\infty} .
$$

We claim that the action of $G$ on the Killing spinors is induced by the action of $\operatorname{Spin}(1, p) \times \operatorname{Spin}(d-p-1)$ on the asymptotic spinors $\varepsilon_{\infty}$. Indeed, consider the action of a Killing vector $\xi$ in the Lie algebra $\mathfrak{g}$ of $G$ on a Killing spinor $\varepsilon=e^{D} \varepsilon_{\infty}$. Since $D$ is constant along the orbits of $\xi$,

$$
\mathcal{L}_{\xi} \varepsilon=e^{D} \mathcal{L}_{\xi} \varepsilon_{\infty}
$$

where we have used the derivation property (1.6) of the Lie derivative. On the other hand, since $\xi$ also preserves $F_{4}$, the Lie derivative of a Killing spinor is again a Killing spinor, whence

$$
\mathcal{L}_{\xi} \varepsilon=e^{D} \varepsilon_{\infty}^{\prime},
$$

for some constant spinor $\varepsilon_{\infty}^{\prime}$. Comparing the two expressions we see that

$$
\mathcal{L}_{\xi} \varepsilon_{\infty}=\varepsilon_{\infty}^{\prime},
$$


which is manifestly independent of $r$. We can therefore compute it in the asymptotic limit $r \rightarrow \infty$ where the spacetime is flat; but in flat space, the action of $\mathcal{L}_{\xi}$ on spinors coincides with the restriction to $\mathfrak{g}$ of the spinor representation of $\operatorname{Spin}(1,10)$. In other words, translations act trivially and a Lorentz transformation $\xi \in \mathfrak{g}$ of the form $\xi=\lambda^{M N} x_{M} \partial_{N}$ acts as

$$
\mathcal{L}_{\xi} \varepsilon_{\infty}=\frac{1}{2} \lambda^{M N} \Sigma_{M N} \varepsilon_{\infty}
$$

where $\Sigma_{M N}$ are the spin generators in the Clifford algebra. Notice that since $\operatorname{dvol}\left(\mathbb{E}^{1, p}\right)$ is $G$-invariant, the action of $G$ on spinors restricts to the subspace defined by (1.8).

Essentially the same argument works for delocalised branes, where the functions $A, B$ and $C$ entering the solution are invariant under translations in the transverse space. This will allow us to also use the methods of [16] in order to study the supersymmetric reductions of delocalised brane solutions. There are however two fundamental differences between flat space and the brane backgrounds. One is the obvious fact that the brane metric is not flat and hence the norm of vector fields will differ. We will see that there exist Killing vector fields which in flat space would have a Killing horizon but which in the spacetime exterior to the brane are everywhere spacelike (except perhaps at the brane horizon). The second difference is reflected in the fact that the symmetries of the brane are a proper subgroup of the symmetries of the flat asymptotic geometry.

These very differences make this problem more interesting than the flat space problem. The existence of spacelike Killing vectors which would have Killing horizons in flat space underlies some of the novel reductions described in this paper. Similarly, in flat space any two (spacelike, say) translations are equivalent under conjugation. This means that we can choose coordinates so that any translation is along one of the coordinates. In contrast, for a (delocalised) brane solution, the symmetry is not large enough to conjugate a translation tangent to the brane into a translation perpendicular to the brane. This means that we can consider translations $a \partial_{\|}+b \partial_{\perp}$ where $\partial_{\|}$ and $\partial_{\perp}$ are the components tangent and perpendicular to the brane, respectively. The Kaluza-Klein reduction along such translations gives a "pencil" of supersymmetric reductions interpolating smoothly between the extremes $a=0$ and $b=0$.

\subsection{Contents and summary of results}

Let us now outline the results and the organisation of the paper. 
Section 2 starts with a brief review of the results of [16], and continues with the main theoretical results which will be applied in the remainder of the paper to study the set of M-theory backgrounds obtained by reducing the M2- and M5-brane configurations along the orbits of a one-parameter subgroup of their isometry groups. We discuss the action of Killing vectors on Killing spinors and two equivalent methods which can be used to determine the loci of supersymmetric reductions. We also derive general formulae for the type IIA fields within the ansätze considered in this paper, which are applied in later sections when we study specific configurations.

Section 3 applies the technology developed in Section 2 to the M2-brane both for the localised solution in Section 3.1 and for the solution which has been delocalised along one transverse direction in Section 3.2. As a consequence of our analysis, we find composite configurations of strings, D2-branes and bound states of strings and D2-branes with both fluxbranes and nullbranes. The allowed supersymmetric configurations are summarised in Tables $3,5,6$. In addition to these configurations, we find new backgrounds obtained through reductions involving timelike and lightlike translations. When the M2-branes are localised, one can use a linear combination of a timelike translation and a nowhere-vanishing transverse rotation to the brane. This is case (A) in Section 3.1. If the M2-branes are delocalised, one can reduce by the action of Killing vectors involving a linear combination of the delocalised direction and a timelike or lightlike direction along the brane. This is case (B) in Section 3.2. In Section 3.2.3 we prove that in this case there are no closed causal curves in the quotient manifold.

Section 4 does for the M5-brane what Section 3 did for the M2-brane. Composite configurations of D4-branes, NS5-branes and bound states D4NS5 with both fluxbranes and nullbranes are found and classified. The results concerning supersymmetric configurations are summarised in Tables 8 , 10 and 11. As in the M2-brane reductions, we find new backgrounds under the same circumstances as stated above, but this time both occur for the delocalised M5-brane: both cases (A) and (B) in Section 4.2 include novel reductions. In Section 4.2.3 we prove that for some of these reductions, the quotient manifold has no closed causal curves.

Section 5 argues why our technology can be straightforwardly applied to many other configurations directly in type IIA/IIB. The duality relation among the configurations obtained in this way and the ones discussed extensively in this paper is explained. We end up with some remarks concerning the gauge theory dual description of some our backgrounds in the context of the AdS/CFT correspondence. 
Some facts about the decomposition of the spinors representation of Spin $(1,10)$ under certain subgroups, needed to determine the amount of supersymmetry preserved by the backgrounds described in the body of the paper, are discussed in Appendix A.

\section{Kaluza-Klein reductions of brane solutions}

In this section we describe some general features of the problem and of the method used in this paper to solve it. The problem of determining the admissible supersymmetric Kaluza-Klein reductions can be essentially mapped to a problem in flat space but with a restricted group of isometries. We will outline our method to classify the supersymmetric Kaluza-Klein reductions of these backgrounds and we will then derive general expressions for the IIA fields obtained by Kaluza-Klein reduction. The results of this section will be used repeatedly in the rest of the paper. In order to ease comparison with the flat case and to make this paper self-contained, we will start the section with a brief review of the flat case.

\subsection{Brief review of flat reductions}

In [16] we classified those Kaluza-Klein reductions of the eleven-dimensional vacuum of M-theory which give rise to smooth ten-dimensional geometries and determined which of those were supersymmetric. Let us rephrase this problem in a way that will facilitate the comparison with the problems treated in this paper. Let $P=\operatorname{ISO}(1,10)$ be the eleven-dimensional Poincaré group and let $\mathfrak{p}$ be the its Lie algebra. We will identify $\mathfrak{p}$ with the Lie algebra of Killing vectors in Minkowski space. We denote $\mathcal{T} \subset \mathfrak{p}$ the subset of $\mathfrak{p}$ consisting of Killing vectors $\xi$ which obey the following properties:

1. $\xi$ is everywhere spacelike; and

2. $\xi$ preserves some nonzero (covariantly) constant spinor.

The subset $\mathcal{T}$ is preserved by the adjoint action of $P$ on $\mathfrak{p}$, which is the action induced on $\mathfrak{p}$ by the geometric action of $P$ on Minkowski space. Similarly, $\mathcal{T}$ is preserved by rescalings $\xi \mapsto s \xi$ for any nonzero real $s \in \mathbb{R}^{\times}$. In [16], we determined the moduli space $\mathcal{M}=\mathcal{T} /\left(P \times \mathbb{R}^{\times}\right)$. There are two families of solutions with three parameters each:

$$
\xi=\partial_{z}+\theta_{1} R_{12}+\theta_{2} R_{34}+\theta_{3} R_{56}+\theta_{4} R_{78}
$$


and

$$
\xi=\partial_{z}+N_{+1}+\theta_{1}^{\prime} R_{34}+\theta_{2}^{\prime} R_{56}+\theta_{3}^{\prime} R_{78},
$$

with $\theta_{1}+\theta_{2}+\theta_{3}+\theta_{4}=0$ and $\theta_{1}^{\prime}+\theta_{2}^{\prime}+\theta_{3}^{\prime}=0$. The notation is that $z$ is the tenth spacelike direction, $R_{i j}$ is the generator of infinitesimal rotations in the $(i j)$-plane, and $N_{+i}$ the generator of an infinitesimal null rotation in the $i$ th direction, where the light-cone coordinates are given by $x^{ \pm}=$ $\left(x^{9} \pm x^{0}\right) / \sqrt{2}$. The former family gives rise to fluxbranes, whereas the latter gives rise to nullbranes and solutions interpolating between nullbranes and fluxbranes. The resulting IIA objects are summarised in Table 1 together with the fraction $\nu$ of the supersymmetry of the vacuum which the solution preserves and the spinor isotropy subalgebra to which the Killing vector belongs. The notation $\mathrm{F} p$ and $\mathrm{N}$ stands for a flux $p$-brane and a nullbrane, respectively; and the objects labelled $\mathrm{F} p / \mathrm{N}$ interpolate between them. In these cases, the fraction of the supersymmetry displayed in the table is the one at generic points in the moduli space: there are lower-dimensional loci where supersymmetry is enhanced.

\begin{tabular}{|c|c|l|}
\hline$\nu$ & Object & Subalgebra \\
\hline \hline$\frac{1}{2}$ & $\mathrm{~F} 5$ & $\mathfrak{s u}(2)$ \\
$\frac{1}{2}$ & $\mathrm{~N}$ & $\mathbb{R}$ \\
$\frac{1}{4}$ & $\mathrm{~F} 3$ & $\mathfrak{s u}(3)$ \\
$\frac{1}{4}$ & $\mathrm{~F} 1$ & $\mathfrak{s p}(2)$ \\
$\frac{1}{4}$ & $\mathrm{~F} 5 / \mathrm{N}$ & $\mathfrak{s u}(2) \times \mathbb{R}$ \\
$\frac{1}{8}$ & $\mathrm{~F} 1$ & $\mathfrak{s u}(4)$ \\
$\frac{1}{8}$ & $\mathrm{~F} 3 / \mathrm{N}$ & $\mathfrak{s u}(3) \times \mathbb{R}$ \\
\hline
\end{tabular}

Table 1: Supersymmetric IIA fluxbranes and nullbranes

Now let $G \subset P$ be a subgroup of the Poincaré group and $\mathfrak{g} \subset \mathfrak{p}$ be the corresponding Lie subalgebra. The subspace $\mathcal{T} \cap \mathfrak{g}$ is now preserved by scaling and by conjugation by $G$, and this prompts us to define the moduli space $\mathcal{M}(G)=(\mathcal{T} \cap \mathfrak{g}) /\left(G \times \mathbb{R}^{\times}\right)$. The problem of determining $\mathcal{M}(G)$ is a restriction of the flat space problem, obtained by restricting the Poincaré group to $G$. As we will see presently, the problem of determining the possible physically distinct supersymmetric Kaluza-Klein reductions of many brane-like solutions, reduces morally to determining $\mathcal{M}(G)$ where $G$ is the symmetry group of the brane-like solution. We say 'morally' because there will be a small subtlety in that we will have to enlarge the space $\mathcal{T} \cap \mathfrak{g}$ to 
include Killing vectors in $\mathfrak{g}$ which are spacelike relative to the brane metric and which may vanish at the horizon. These vector fields will not belong to $\mathcal{T}$ but to some larger subspace of the Poincare algebra which will depend on the particular brane solution (e.g., on the charge) and not simply on the symmetry group. It is precisely this fact which prevents us from claiming that the problem of determining the possible supersymmetric Kaluza-Klein reductions of M-branes reduces precisely to the flat case; although it does so to a large extent.

\subsection{Methodology}

Let us recall our aims and outline the method we have followed to achieve them.

Given a subgroup $G \subset \operatorname{ISO}(1,10)$ of symmetries of an M-theory background $\left(M, g, F_{4}\right)$ of type (1.7), we would like

1. to classify the one-dimensional (connected) subgroups $\Gamma \subset G$ acting freely on $M$ with spacelike orbits and leaving invariant some nonzero Killing spinor; and

2. for every such subgroup, to write down explicitly the reduced IIA background.

In the following section we will address (2) by giving general formulas for the IIA fields, but let us first restate (1) using the observations made above.

Let $\mathfrak{g}$ be the Lie algebra of $G$, which we identify with the Killing vectors generating its action on $M$. As a first step we will determine those vectors $\xi \in \mathfrak{g}$ which are spacelike (at least outside the brane horizon). This in effect results in the classification of reductions which are not necessarily supersymmetric or even smooth. Doing so requires a careful yet elementary analysis of the norm of a Killing vector relative to the brane metric. A perhaps surprising result born out of this analysis is the existence of everywhere spacelike Killing vectors which on flat space would not have this property.

Having determined the everywhere spacelike Killing vectors we must ensure that the action they generate is free: being spacelike means that they never vanish, whence the action is locally free. To ensure that every point has trivial stabiliser is equivalent to demanding the presence of the translation component in the Killing vector. Since we are interested in the moduli space $\mathcal{M}$ of smooth supersymmetric Kaluza-Klein reductions, we are free to 
conjugate by the action of $G$; that is, to choose appropriate coordinates by performing a symmetry transformation.

Finally we must impose that $\xi$ preserve some nonzero Killing spinor in the background. The same argument as in Section 1.4 shows that the action of $\xi$ on spinors coincides with the action of $\mathfrak{g}$ on the spinor representation of $\operatorname{Spin}(1,10)$. This reduces the problem of finding supersymmetric KaluzaKlein reductions of solutions of the type (1.7) to a flat space problem with a restricted Poincare group. Furthermore, we must ensure that the invariant spinors satisfy equation (1.8). Since translations act trivially on spinors, only the Lorentz component of $\xi$ is constrained. In all cases, this component is either a rotation or a commuting linear combination of a rotation and a null rotation, and as we will now outline, supersymmetry will only constrain the rotation. Two methods can be used to determine the supersymmetric loci: a representation-theoretical method based on the weight decomposition of various spinorial representations, and a direct computational method based on the Clifford algebra. Since both have their merits, we will describe them both.

\subsubsection{Representation-theoretical method}

Let $S$ be the unique half-spin representation of the eleven-dimensional spin group. (In Appendix A this representation will be denoted $S_{11}$ to emphasise its eleven-dimensional origin.) The Clifford algebra can act on $S$ in one of two inequivalent ways, but as mentioned above we will assume that a choice has been made once and for all. (This choice is dictated by the supersymmetry transformation laws, equivalently by the Killing spinor equation.) The Killing spinors of the background are in one-to-one correspondence with a linear subspace $S_{0}$ of $S$ determined by some $G$-equivariant equations of the form (1.8). Equivariance simply means that the action of $G$, and hence that of $\xi$, restricts to $S_{0}$. We would like to determine for which $\xi$ are there $\xi$ invariant spinors in $S_{0}$. As mentioned in the previous paragraph, only the Lorentz component $\lambda$ acts nontrivially on spinors, and in all the cases we will consider this will be of the form $\lambda=\nu+\rho$, where the null rotation $\nu$ and the rotation $\rho$ commute. Since $\nu$ is nilpotent and $\rho$ semisimple, $\lambda$ annihilates a spinor if and only if it is annihilated by both $\nu$ and $\rho$ separately (cf., the argument in [16, Section 2.2]). Since $\nu$ and $\rho$ commute, the subspace of $\rho$-invariant spinors in $S_{0}$ is preserved by $\nu$. Now, without loss of generality, $\nu$ acts on spinors as a multiple of $\Gamma_{+} \Gamma_{1}$, say, and both $\Gamma_{+}$and $\Gamma_{-}$commute with $\rho$. Because $\Gamma_{+} \Gamma_{-}+\Gamma_{-} \Gamma_{+}$is proportional to the identity, the subspace of $\rho$-invariant spinors in $S_{0}$ is even-dimensional and breaks up into two equidimensional subspaces consisting of spinors annihilated by $\Gamma_{+}$ 
(hence by $\nu$ ) and by $\Gamma_{-}$. Therefore only $\rho$ is constrained by the existence of invariant spinors and the presence of the null rotation is only reflected in a further halving of the fraction of supersymmetry which the reduction preserves.

Let us now discuss the constraints on $\rho$ arising out of the existence of $\rho$-invariant spinors in $S_{0}$. The rotation $\rho$ belongs to the maximal compact subalgebra $\mathfrak{k}$ of $\mathfrak{g}$ and hence in some fixed Cartan subalgebra $\mathfrak{h}$ of $\mathfrak{k}$. Up to isomorphism, the action of $\mathfrak{h}$ on $S_{0}$ is completely specified by giving the weights together with their multiplicities. Weights are linear functionals on $\mathfrak{h}$ hence the existence of $\rho$-invariant spinors is equivalent to there being some weight $\mu$ which annihilates $\rho$. The rotation $\rho$ defines a point in $\mathfrak{h}$ with coordinates $\boldsymbol{\theta}=\left(\theta_{1}, \ldots, \theta_{\ell}\right)$ and the condition that a weight $\mu$ annihilates $\rho$ translates into a homogeneous linear equation on $\boldsymbol{\theta}$. The solutions of this equation will define a hyperplane in $\mathfrak{h}$. Doing this for all the weights ${ }^{4}$ we obtain a family of hyperplanes defining the locus of supersymmetric reductions. The bigger the number of these hyperplanes a rotation $\rho$ belongs to, the more supersymmetry will the reduction preserve.

It is then a simple matter to determine the weight decomposition of $S_{0}$ for each of the M-theory backgrounds discussed in this paper and determine the locus of supersymmetry reductions for each one. The necessary group theory is outlined in Appendix A.

\subsubsection{Direct method}

Alternatively one can obtain the same result by directly solving the algebraic equation (1.9). The most general form of the latter, that is going to be used in this paper, can be written as

$$
\left(\alpha \mathcal{P}_{+}+\sum_{i=1}^{n} \beta_{i} \mathcal{P}_{i}\right) \varepsilon_{\infty}=0,
$$

where $\mathcal{P}_{+}$and $\mathcal{P}_{i}$ are commuting linear transformations on spinors obeying $\mathcal{P}_{+}^{2}=0$ and $\mathcal{P}_{\imath}^{2}=-\mathbb{1}$ for all $i .^{5}$

The above equation defines two linear transformations $N=\alpha \mathcal{P}_{+}$and

\footnotetext{
${ }^{4}$ Due to the freedom to conjugate by $G$, we need only consider one weight in each Weyl orbit.

${ }^{5}$ Notice that when one considers such an expression, the freedom under conjugation by the isometry group has already been taken into account, as explained in more detail for the different configurations studied in this paper. In particular, one can think of $\mathcal{P}_{+}=\Gamma_{+1}$ and $\mathcal{P}_{\imath}=\Gamma_{\imath, \imath+1}$.
} 
$S=-\sum_{i=1}^{n} \beta_{i} \mathcal{P}_{i}$

$$
N \varepsilon_{\infty}=S \varepsilon_{\infty}
$$

$N$ being nilpotent and $S$ semisimple. Using the same argument of [16, Section 2.2], the original equation decomposes into

$$
N \varepsilon_{\infty}=0 \quad \text { and } \quad S \varepsilon_{\infty}=0 .
$$

The first equation breaks one half of the supersymmetry, and it is associated with null rotations. Let us now discuss the solutions to the second equation in (2.2). By squaring it, one derives the further constraint

$$
\left(\sum_{i<j} \alpha_{i j} Q_{i j}\right) \varepsilon_{\infty}=\mathcal{K} \varepsilon_{\infty}
$$

where $Q_{i j}=\mathcal{P}_{i} \mathcal{P}_{j}, \alpha_{i j}=2 \beta_{i} \beta_{j}$ and $\mathcal{K}=-\sum_{i=1}^{n}\left(\beta_{i}\right)^{2}$. Notice that the $\mathcal{Q}_{i j}$ are also a commuting family of linear transformations.

Assuming that the parameter $\beta_{i}$ do not satisfy any relations, the general solution to the above equation is given by

$$
Q_{i j} \varepsilon_{\infty}=\eta_{i j} \varepsilon_{\infty} \quad \mathcal{K}=\sum_{i<j} \eta_{i j} \alpha_{i j}
$$

for some signs $\eta_{i}$. This is easily shown by induction on the number of pairs $m=\left(\begin{array}{l}n \\ 2\end{array}\right)$ determining the number of matrices $Q_{i j}$. Indeed, for $m=1$ (equivalently, $n=2)$ it is trivial to prove that $\mathcal{K}=\eta_{12} \alpha_{12}$ and $Q_{12} \varepsilon_{\infty}=\eta_{12} \varepsilon_{\infty}$ is the solution. Assuming, our result is true for a given $m$, we shall now show the result also holds for $m+1$. Indeed, the starting equation can be decomposed as

$$
\left(\left(\sum_{i=1}^{m} \alpha_{(i)} \mathcal{Q}_{(i)}\right)+\alpha_{(m+1)} \mathcal{Q}_{(m+1)}\right) \varepsilon_{\infty}=\mathcal{K}_{(m+1)} \varepsilon_{\infty} .
$$

By assumption, it can just be rewritten as

$$
\alpha_{(m+1)} \mathcal{Q}_{(m+1)} \varepsilon_{\infty}=\left(\mathcal{K}_{(m+1)}-\mathcal{K}_{(m)}\right) \varepsilon_{\infty},
$$

which is solved by

$$
\begin{gathered}
\mathcal{Q}_{(m+1)} \varepsilon_{\infty}=\eta_{(m+1)} \varepsilon_{\infty}, \quad \text { and } \\
\mathcal{K}_{(m+1)}=\mathcal{K}_{(m)}+\eta_{(m+1)} \alpha_{(m+1)} .
\end{gathered}
$$


Thus we obtain the full solution for $m+1$.

It is important to stress that not all conditions on the asymptotic Killing spinors are independent. Indeed, there are only $n-1$ independent conditions

$$
\mathcal{P}_{1} \varepsilon_{\infty}=-\eta_{1 \jmath} \mathcal{P}_{j} \varepsilon_{\infty} \quad, \quad j=2, \ldots, n
$$

since it is trivial to show that $\eta_{j j^{\prime}}=-\eta_{1 j} \eta_{1 \jmath^{\prime}}$ for all $2 \leq j \neq j^{\prime} \leq n$. Inserting these relations into the solution for the eigenvalue $\mathcal{K}$, and solving for $\beta_{i}$, one derives the constraint on the parameters determining the infinitesimal isometry:

$$
\beta_{1}=\sum_{j=2}^{n} \eta_{1 j} \beta_{j}
$$

With all this information, it is straightforward to show that the original second equation in (2.2) is satisfied.

As emphasised before, it is not claimed that the above solution is the most general one. Indeed, whenever the coefficients $\beta_{i}$ satisfy certain relations among them, there may be an enhancement of supersymmetry. It is actually very simple to argue when such a phenomena is going to happen. Indeed, whenever we have a linear combination of matrices annihilating a vector, the annihilation still holds if a subset of the matrices already annihilate the vector by themselves. Thus, there are as many solutions as different ways of decomposing the original linear combination compatible with the existence of a solution. Instead of developing the general theory for arbitrary $n$, we shall just state the results needed in the bulk of this paper. For our purposes, $n \leq 5$ (since there are ten spacelike directions). It is obvious that $n=1$ breaks supersymmetry completely. For $n=2$, the unique solution is the one specified by (2.2). It corresponds to an $\mathfrak{s u}(2)$ subalgebra. For $n=3$, equation (2.2) is still the general solution, since all the decompositions into different subsets break supersymmetry. This case corresponds to the $\mathfrak{s u}(3)$ subalgebra. For $n=4$, there is the generic solution given in (2.2), corresponding to $\mathfrak{s u}(4)$, but there is also the possibility of decomposing $n=n_{1}+n_{2}=2+2$, which corresponds to the $\mathfrak{s u}(2) \times \mathfrak{s u}(2)$ subalgebra. This case arises when $\beta_{1}=\eta_{2} \beta_{2}$ and $\beta_{3}=\eta_{4} \beta_{4}$. Finally, for $n=5$, there is the standard $\mathfrak{s u}(5)$ subalgebra solution (2.2), but also the $\mathfrak{s u}(2) \times \mathfrak{s u}(3)$ one. The latter takes place whenever $\beta_{1}=\eta_{2} \beta_{2}$ and $\beta_{3}=\eta_{4} \beta_{4}+\eta_{5} \beta_{5}$. It is understood that depending on the isometry group of the background under consideration, given a decomposition $n=\sum_{i} n_{i}$, there might be more than one inequivalent configuration associated with it. 


\subsection{Explicit formulas for the Kaluza-Klein reduction}

We now give a general formula for the Kaluza-Klein reduction of the metric and the four-form. This is facilitated by going to adapted coordinates where the Killing vector $\xi$ is simply a translation. As in [16] we will exhibit $\xi$ as a "dressed" translation, which yields at once the required change of coordinates.

\subsubsection{Kaluza-Klein reduction of the metric}

In this section we give a general formula for the Kaluza-Klein reduction of the metric. Let us assume that the eleven-dimensional metric in the coordinate system $(z, \boldsymbol{y})$, where the Killing vector being used in the reduction is $\xi=\partial_{z}+\alpha$, can be written as ${ }^{6}$

$$
g=\sum_{i} V_{i}(\boldsymbol{y})^{\alpha_{2}} d s^{2}\left(\mathbb{E}^{i}\right)+V^{\gamma}(\boldsymbol{y})(d z)^{2} .
$$

Ansatz (2.4) includes the backgrounds discussed in this paper. Furthermore, $\alpha$ is an affine transformation of the Cartesian coordinates $(\boldsymbol{y})$. It is important to stress that in later applications, $\alpha$ will always be a linear combination of commuting infinitesimal transformations commuting with $\partial_{z}$.

It is useful to introduce some set of projectors $\mathbb{P}_{i}$ satisfying, for all $i$,

$$
d s^{2}\left(\mathbb{E}^{i}\right)=(d \boldsymbol{y})^{t} \mathbb{P}_{i} \eta d \boldsymbol{y}
$$

where $\eta$ stands for the ten-dimensional Minkowski metric. As in [16], the description of the explicit geometry obtained through the reduction along the orbits of the Killing vectors is obtained by working in coordinates $(z, x)$ adapted to the Killing vector, $\xi=\partial_{z}$. The explicit change of coordinates is obtained by noticing that $\xi$ is simply a dressed version of its translation component

$$
\xi=U \partial_{z} U^{-1} \quad \text { where } \quad U=\exp (-z \alpha)
$$

Thus, defining

$$
\boldsymbol{x}=U \boldsymbol{y},
$$

\footnotetext{
${ }^{6}$ In the bulk of the paper, we shall also deal with configurations in which the $z$ coordinate is also either a timelike or a lightlike coordinate. It is straightforward to extend the formalism developed below to these cases.
} 
it follows that $\xi \boldsymbol{x}=0$, so that $\boldsymbol{x}$ are good coordinates for the space of orbits. Since $\alpha$ is a linear combination of infinitesimal Lorentz transformations and translations, its action on $\boldsymbol{y}$ can be defined by

$$
\alpha \boldsymbol{y}=B \boldsymbol{y}+\boldsymbol{C},
$$

where $B$ is, generically, a $10 \times 10$ constant matrix, whereas $C$ is a constant 10 -vector taking care of the inhomogeneous part of the infinitesimal transformation generated by $\alpha$. Thus, $\boldsymbol{x}(z, \boldsymbol{y})=e^{-z B}\left(\boldsymbol{y}+B^{-1} \boldsymbol{C}\right)-B^{-1} \boldsymbol{C}$, so that

$$
d \boldsymbol{y}=e^{z B}[d \boldsymbol{x}+(B \boldsymbol{x}+\boldsymbol{C}) d z] .
$$

We can now rewrite the metric (2.4) in the adapted coordinate system $(z, \boldsymbol{x})$, obtaining

$$
g=\Lambda(d z+A)^{2}-\Lambda A^{2}+\sum_{i} V_{i}(\boldsymbol{y})^{\alpha_{2}}(\boldsymbol{x})(d \boldsymbol{x})^{t} \mathbb{P}_{i} \eta d \boldsymbol{x},
$$

where

$$
\begin{aligned}
& \Lambda=V^{\gamma}(\boldsymbol{x})+\sum_{i} V_{i}(\boldsymbol{y})^{\alpha_{2}}(\boldsymbol{x})(B \boldsymbol{x}+\boldsymbol{C})^{t} \mathbb{P}_{i} \eta(B \boldsymbol{x}+\boldsymbol{C}) \\
& A=\Lambda^{-1} \sum_{i} V_{i}(\boldsymbol{y})^{\alpha_{2}}(\boldsymbol{x})(B \boldsymbol{x}+\boldsymbol{C})^{t} \mathbb{P}_{i} \eta d \boldsymbol{x} .
\end{aligned}
$$

Using the Kaluza-Klein ansatz (1.1) we can read off the dilaton $\Phi=$ $\frac{3}{4} \log \Lambda$ and the IIA metric

$$
g=\Lambda^{1 / 2}\left\{\sum_{i} V_{i}(\boldsymbol{y})^{\alpha_{2}}(\boldsymbol{x})(d \boldsymbol{x})^{t} \mathbb{P}_{i} \eta d \boldsymbol{x}-\Lambda A^{2}\right\},
$$

whereas the RR 1-form is given by (2.7).

\subsubsection{Kaluza-Klein reduction of the four-form and its dual}

We now give a general formula for the Kaluza-Klein reduction of the fourform $F_{4}$ and its dual. In the $(z, y)$ coordinates we can write the four-form $F_{4}$ uniquely as

$$
F_{4}(z, \boldsymbol{y})=K(z, \boldsymbol{y})-d z \wedge L(z, \boldsymbol{y})
$$


where $K$ and $L$ are a four-form and three-form, respectively, without $d z$ components. Let us change coordinates to $(z, \boldsymbol{x})$. By the results of Section 1.2 we know that the resulting expression for $F_{4}$ is given by equation (1.2), where the forms $H_{4}$ and $H_{3}$ are basic. This means that in the adapted coordinate system $(z, \boldsymbol{x})$ they do not depend explicitly on $z$ nor do they have any component in $d z$. We can exploit this fact in order to give an explicit expression for $H_{3}$ and $H_{4}$ in terms of the forms $K$ and $L$ in (2.9). The idea is simple: we perform the explicit change of coordinates in (2.9) and write the result in the form (1.2). To simplify the calculation we set $z=0$, since we know a priori that the resulting forms do not depend on $z$. We find that

$$
\left.K(z, \boldsymbol{y}(z, \boldsymbol{x}))\right|_{z=0}=K(0, \boldsymbol{x})+d z \wedge \imath_{B \boldsymbol{x}+\boldsymbol{C}} K(0, \boldsymbol{x}),
$$

and similarly for $L$, where we have used that at $z=0, \boldsymbol{y}(0, \boldsymbol{x})=\boldsymbol{x}$. Inserting this into (2.9) and comparing with (1.2) we find

$$
H_{4}(\boldsymbol{x})=K(0, \boldsymbol{x}) \quad \text { and } \quad H_{3}(\boldsymbol{x})=L(0, \boldsymbol{x})-\imath_{B \boldsymbol{x}+\boldsymbol{C}} K(0, \boldsymbol{x}) .
$$

The same method also works mutatis mutandis for the seven-form $F_{7}$ dual to $F_{4}$, and indeed for any invariant $p$-form. In some backgrounds it is more convenient to work with $F_{7}$ and use the above method to determine the forms $H_{7}$ and $H_{6}$ in (1.3), from which we can then recover the forms $H_{3}$ and $H_{4}$ using the duality relations (1.5).

\section{Kaluza-Klein reductions of the M2-brane}

In this section we classify the set of M-theory backgrounds obtained by modding out the M2-brane background by a one-parameter subgroup of its isometry group and study the smooth supersymmetric Kaluza-Klein reductions along the orbits of the Killing vectors generating such subgroups. We shall first consider the standard M2-brane configuration in section 3.1. Afterwards, we shall discuss the M2-brane delocalised along one transverse direction in section 3.2 .

\subsection{Supersymmetric reductions of the M2-brane}

The M-theory membrane [76] is described by a metric of the type (1.7) with two factors,

$$
g=V^{-2 / 3} d s^{2}\left(\mathbb{E}^{1,2}\right)+V^{1 / 3} d s^{2}\left(\mathbb{E}^{8}\right)
$$


where $V=1+|Q| / r^{6}$ with $|Q|$ some positive constant and $r$ the radial distance in the transverse $\mathbb{E}^{8}$. The 4 -form is given by

$$
F_{4}=\operatorname{dvol}\left(\mathbb{E}^{1,2}\right) \wedge d V^{-1},
$$

up to a constant of proportionality. The Killing spinors are of the form

$$
\varepsilon=V^{-1 / 6} \varepsilon_{\infty},
$$

where $\varepsilon_{\infty}$ is a constant spinor satisfying

$$
\operatorname{dvol}\left(\mathbb{E}^{1,2}\right) \cdot \varepsilon_{\infty}=\varepsilon_{\infty}
$$

The symmetry group is

$$
G=\operatorname{ISO}(1,2) \times \mathrm{SO}(8) \subset \operatorname{ISO}(1,10),
$$

with Lie algebra

$$
\mathfrak{g}=\left(\mathbb{R}^{1,2} \rtimes \mathfrak{s o}(1,2)\right) \times \mathfrak{s o}(8),
$$

whence any Killing vector $\xi$ can be decomposed as

$$
\xi=\tau_{\|}+\lambda_{\|}+\rho_{\perp}
$$

where, mnemonically, $\tau, \lambda$ and $\rho$ denote a translation, a Lorentz transformation and a rotation, respectively, and where the subscripts $\|$ and $\perp$ refer to vector fields tangent and perpendicular to the brane worldvolume, respectively. We will often omit these subscripts if doing so does not result in ambiguity.

\subsubsection{Freely-acting spacelike isometries}

The geometrical action of $G$ on the coordinates induces an action on the Killing spinors which translates into conjugation by $G$ on the Lie algebra $\mathfrak{g}$. Using this freedom, we may bring $\lambda$ into a normal form. Nontrivial Lorentz transformations in $\mathfrak{s o}(1,2)$ come in three flavours depending on the type of vector in $\mathbb{E}^{1,2}$ that they leave invariant. Therefore either $\lambda=0$ or, via a Lorentz transformation in the worldvolume of the membrane, it can be brought to one of the following three normal forms:

1. $\lambda$ fixes a timelike vector:

$$
\lambda=\theta R_{12} \quad \theta \neq 0,
$$


2. $\lambda$ fixes a spacelike vector:

$$
\lambda=\beta B_{02} \quad \beta \neq 0,
$$

3. $\lambda$ fixes a null vector:

$$
\lambda=N_{+2}
$$

where $R_{12}$ is the generator of infinitesimal rotations in the (12)-plane, $N_{+2}$ is the generator of infinitesimal null rotation in the $x^{2}$ direction with lightcone coordinates $x^{ \pm}=\left(x^{1} \pm x^{0}\right) / \sqrt{2}$, and where $B_{02}$ is the generator of infinitesimal boosts along the $x^{2}$ direction.

We can now use the freedom to change origin in the worldvolume of the brane- equivalently, to conjugate by the translation subgroup in $\operatorname{ISO}(1,2)$ in order to bring $\tau$ to a normal form. If $\lambda=0, \tau$ does not change; but in the other normal forms we can bring $\tau$ to the following: (1) $\tau \propto \partial_{0}$, (2) $\tau \propto \partial_{1}$, and (3) $\tau \propto \partial_{-}$. It is easy to see that in case (2) there are points outside the brane horizon where $\xi$ is timelike, hence this case is ruled out. It is also easy to see that in case (3) we must have $\tau=0$ for precisely the same reasons. This narrows down the possibilities to three cases:

(a) $\xi=\tau+\rho_{\perp}$, with $\tau$ so far unconstrained;

(b) $\xi=\tau+\rho_{\|}+\rho_{\perp}$, with $\tau$ timelike and orthogonal to $\rho_{\|}$; and

(c) $\xi=\nu_{\|}+\rho_{\perp}$, with $\nu_{\|}$a null rotation.

In the above expressions $\rho_{\perp}$ is an infinitesimal rotation in $\mathfrak{s o}(8)$ and hence can be brought to a normal form

$$
\rho_{\perp}=\theta_{1} R_{34}+\theta_{2} R_{56}+\theta_{3} R_{78}+\theta_{4} R_{9 \natural} .
$$

We must distinguish between two cases: either one or more of the $\theta$ s vanish or none does. If some $\theta \mathrm{s}$ vanish, only case (a) above gives rise to a spacelike $\xi$ and in that case we must take $\tau$ to be spacelike. If none of the $\theta$ s vanish, we have more possibilities: case (c) can occur, and so can cases (a) and (b) provided that $\|\tau\|^{2}$ is not too negative. To understand this, let us compute the norm of $\xi$ in these cases.

Consider a Killing vector of the form $\xi=\tau+\rho_{\|}+\rho_{\perp}$, where $\tau$ is orthogonal to $\rho_{\|}$and where we allow $\rho_{\|}$to be zero. In this way we can discuss cases (a) and (b) simultaneously. The norm of this vector field relative to the membrane metric is given by

$$
\|\xi\|^{2}=V^{-2 / 3}\left(\|\tau\|_{\infty}^{2}+\left\|\rho_{\|}\right\|_{\infty}^{2}\right)+V^{1 / 3}\left\|\rho_{\perp}\right\|_{\infty}^{2}
$$


where $\|\cdot\|_{\infty}$ is the norm relative to the flat metric. The tangent vector $\rho_{\perp}$ at a point a distance $r>0$ away from the membrane is tangent to the transverse sphere of radius $r$ through that point. This means that the norm at that point is given by

$$
\left\|\rho_{\perp}\right\|_{\infty}^{2}=r^{2}\left\|\rho_{\perp}\right\|_{S}^{2}
$$

where $\|\cdot\|_{S}$ is the norm relative to the round metric on the sphere of unit radius. Since the sphere is compact, $\left\|\rho_{\perp}\right\|_{S}$ acquires a maximum and a minimum, whence

$$
m^{2} r^{2} \leq\left\|\rho_{\perp}\right\|_{\infty}^{2} \leq M^{2} r^{2}
$$

for some non-negative real numbers $m \leq M$. In fact, it is easy to see that for $\rho_{\perp}$ given in (3.11), these numbers are given by

$$
m^{2}=\min _{i} \theta_{i}^{2} \quad \text { and } \quad M^{2}=\max _{i} \theta_{i}^{2} .
$$

It should be stressed that both inequalities in (3.12) are sharp, since there are directions (i.e., points in the sphere) where the inequalities are saturated. The lower bound $m$ is positive if and only if $\rho_{\perp}$ does not leave any directions invariant. This is possible for the M2-brane since the transverse sphere is odd-dimensional, for on an even-dimensional sphere every (continuous) vector field has a zero (in fact, two) and hence $m=0$ in those cases. The norm of $\xi$ is then bounded below by

$$
\|\xi\|^{2} \geq V^{-2 / 3}\|\tau\|_{\infty}^{2}+V^{-2 / 3}\left\|\rho_{\|}\right\|^{2}+V^{1 / 3} r^{2} m^{2},
$$

and again this inequality is sharp. The rotation $\rho_{\|}$has a zero at the 'origin' of the membrane worldvolume. We can thus simplify the above bound even further:

$$
\|\xi\|^{2} \geq V^{-2 / 3}\|\tau\|_{\infty}^{2}+V^{1 / 3} r^{2} m^{2}
$$

which is still sharp. The right-hand side of the above equation is a function of $r$ and it will attain a minimum at a critical radius $r_{0}$, which is zero if $\|\tau\|_{\infty}^{2} \geq 0$ and positive if $\|\tau\|_{\infty}^{2}<0$. Indeed, the lower bound for the norm of $\xi$ is given by the function

$$
f(r)=V^{-2 / 3}\|\tau\|_{\infty}^{2}+V^{1 / 3} r^{2} m^{2},
$$

whose derivative is given by

$$
f^{\prime}(r)=V^{-5 / 3} \frac{2|Q|}{r^{7}}\left(2\|\tau\|_{\infty}^{2}+m^{2} r^{2}\left(1+\frac{r^{6}}{|Q|}\right)\right) .
$$


This function has a critical point at $r=0$ and at the positive root $r_{0}$ of the equation

$$
V(r) r^{8}=-\frac{2|Q|}{m^{2}}\|\tau\|_{\infty}^{2}
$$

should such a root exist. For $\|\tau\|_{\infty}^{2} \geq 0$, no such root exists and the minimum of $f$ is at $r=0$, whereas for $\|\tau\|_{\infty}^{2}<0$, the minimum is at $r_{0}$. In any case we have the bound

$$
\|\xi\|^{2} \geq V^{-2 / 3}\left(r_{0}\right)\|\tau\|_{\infty}^{2}+V^{1 / 3}\left(r_{0}\right) r_{0}^{2} m^{2},
$$

which is still sharp. The right-hand side is positive for all

$$
\|\tau\|_{\infty}^{2}>-\frac{3}{2} m^{2}(2|Q|)^{1 / 3}
$$

This means that we can allow for $\tau$ to be timelike (but not too much) and still obtain a spacelike Killing vector. If $\rho_{\perp}$ fixes some directions, so that $m=0$, we see that $\tau$ must be spacelike.

In summary, the following Killing vectors in $\mathfrak{g}$ are spacelike:

(A) $\xi=\tau_{\|}+\rho_{\|}+\rho_{\perp}$, with $\rho_{\perp}$ without fixed directions and $\tau$ obeying a constraint on the norm: $\|\tau\|_{\infty}^{2}>-\mu^{2}$, where $\mu$ can be read from equation (3.13), and where we can also allow for $\rho_{\|}=0$ in this case;

(B) $\xi=\tau_{\|}+\rho_{\perp}$, with $\rho_{\perp}$ fixing some directions and $\tau$ spacelike;

(C) $\xi=\nu_{\|}+\rho_{\perp}$, with $\rho_{\perp}$ without fixed directions.

It is clear that in cases (A) (with $\tau \neq 0$ ) and (B), $\xi$ integrates to a free action of a subgroup $\mathbb{R} \subset G$, since $\xi$ contains a translation. The absence of translations in case (C) makes it different from (A) and (B). Even though the action is locally free (for $r>0$ ), one can prove that there are points with nontrivial stabilisers, so that the action is not free and the quotient is therefore singular. Consider, for example, the point $P$ with coordinates $x^{ \pm}=x^{2}=x^{3}=\cdots=x^{9}=0$ and $x^{\natural}=1$. The orbit of this point under the action generated by the Killing vector $\xi=\nu_{\|}+\rho_{\perp}$, with $\rho_{\perp}$ given by (3.11) with all $\theta$ s different from zero, is $x^{ \pm}=x^{2}=x^{3}=\cdots x^{8}=0, x^{9}(t)=\cos \theta_{4} t$ and $x^{\natural}(t)=\sin \theta_{4} t$. As a result, the point $P$ is mapped to itself by those points $t=2 \pi n / \theta_{4}$, for any $n \in \mathbb{Z}$. This defines a subgroup isomorphic to $\mathbb{Z}$ in the $\mathbb{R}$ subgroup generated by $\xi$. Reducing by $\xi$ would therefore result in singularities outside the horizon and so we discard it and with it case (C). A very similar argument would allow us to discard case (A) with $\tau=0$. This leaves us with two possibilities for a freely-acting spacelike vector field: case (A) with $\tau \neq 0$ and case (B). 


\subsubsection{Moduli space of smooth reductions}

Let us now identify more precisely the moduli space of smooth reductions in each of these cases.

In case (A) we must distinguish between two cases, depending on whether or not $\rho_{\|}$vanishes. If $\rho_{\|} \neq 0$, then by changing the origin we can put $\tau=a \partial_{0}$, where $0<a^{2}<\mu^{2}$, with $\mu$ given in (3.13). In summary, $\xi$ can be written in the following form

$$
\xi=a \partial_{0}+\theta_{1} R_{12}+\theta_{2} R_{34}+\theta_{3} R_{56}+\theta_{4} R_{78}+\theta_{5} R_{9 \natural},
$$

where none of the $\theta$ s vanish and $0<|a|<\mu$. The moduli space is obtained from this space by projectivising and by some discrete identifications coming from the action of the Weyl group. Its dimension is therefore fivedimensional, and we will see below that supersymmetry will select a fourdimensional locus.

If $\rho_{\|}=0$, the causal character of the translation is not fixed, although the norm constraint (3.13) is still in force. We must distinguish between three cases, depending on whether $\tau$ is timelike, spacelike or null. If $\tau$ is timelike we can choose coordinates such that $\tau=a \partial_{0}$ with $0<|a|<\mu$. Similarly, if $\tau$ is spacelike, it can be arranged that $\tau=a \partial_{1}$ with $a \neq 0$ but otherwise unconstrained. In either case we have a four-dimensional moduli space. Finally if $\tau$ is null, coordinates can be chosen where $\tau=\partial_{+}$(no free parameter!) whence the moduli space is three-dimensional. In all cases, supersymmetry will select a codimension-one locus.

Finally, in case (B) we can arrange for $\tau=a \partial_{1}$ and hence

$$
\xi=a \partial_{1}+\theta_{2} R_{34}+\theta_{3} R_{56}+\theta_{4} R_{78}
$$

where $a \neq 0$ is not otherwise constrained. The moduli space in this case is only three-dimensional, with supersymmetry selecting a two-dimensional locus.

\subsubsection{Supersymmetry}

Now we impose the condition that the reduction preserves supersymmetry. As explained in Section 2.2, supersymmetry only constrains the rotation component of the Killing vector $\xi$ to lie in the isotropy of some spinor satisfying (3.4). In all the cases above, the rotation component of $\xi$ takes the general form

$$
\rho=\theta_{1} R_{12}+\theta_{2} R_{34}+\theta_{3} R_{56}+\theta_{4} R_{78}+\theta_{5} R_{9 \natural},
$$


with perhaps some of the $\theta$ s vanishing. We find it convenient to treat the general case first, which will give some relations between the $\theta \mathrm{s}$ and then impose any further conditions.

As discussed in Appendix A, the condition that $\rho$ annihilates a Killing spinor is equivalent to $\rho$ being annihilated by some weight in the subspace $S_{0}$ of the half-spin representation defined by (3.4). These weights are given in equation (A.2) with the negative sign, according to our conventions. Therefore a weight will annihilate $\rho$ if and only if the $\theta_{i}$ belong to the union of the following eight hyperplanes

$$
\sum_{i=1}^{5} \mu_{i} \theta_{i}=0 \quad \text { where } \mu_{i}^{2}=1 \text { with } \mu_{2} \cdots \mu_{5}=-1 .
$$

Notice that if $\rho$ is annihilated by $\mu$ it is also annihilated by $-\mu$ which is also a weight in the representation $S_{0}$ : this explains why there are only eight hyperplanes in the above family. If $\rho$ belongs to one and only one such hyperplane the amount of supersymmetry preserved by such a reduction is $\nu=\frac{1}{16}$. This corresponds to $\rho$ belonging to an $\mathfrak{s u}(5)$ subalgebra of $\mathfrak{s o}(1,10) .^{7}$

There is enhancement of supersymmetry if $\rho$ belongs to the intersection of two or more hyperplanes in (3.17). Assuming that none of the $\theta \mathrm{s}$ are zero, we can only have simple intersections between two hyperplanes, e.g., $\theta_{4}=\theta_{5}$ and $\theta_{1}+\theta_{2}+\theta_{3}=0$. This corresponds to a $\rho$ which belongs to an $\mathfrak{s u}(2) \times \mathfrak{s u}(3)$ subalgebra. In this case there are four weights which annihilate $\rho$ and hence the fraction of the supersymmetry preserved by the reduction is enhanced to $\nu=\frac{1}{8}$.

If $\rho_{\|}=0$ then $\theta_{1}=0$, and if the remaining $\theta \mathrm{s}$ do not vanish, the supersymmetric locus is given by the intersection of the hyperplane $\theta_{1}=0$ with the hyperplanes in (3.17). This is a family of four hyperplanes in the $\theta_{1}=0$ subspace, given by the equations

$$
\sum_{i=2}^{5} \mu_{i} \theta_{i}=0 \quad \text { where } \mu_{i}^{2}=1 \text { and } \mu_{2} \cdots \mu_{5}=-1 .
$$

A generic $\rho$ in one of these hyperplanes is annihilated by four weights, hence the reduction preserves a fraction $\nu=\frac{1}{8}$ of the supersymmetry, corresponding to $\rho$ in an $\mathfrak{s u}(4)$ subalgebra. There is again supersymmetry enhancement

\footnotetext{
${ }^{7}$ More precisely, the phrase " $\rho$ belongs to an $\mathfrak{s u}(5)$ subalgebra" is to be interpreted as meaning that $\rho$ belongs to the intersection of an $\mathfrak{s u}(5)$ subalgebra of $\mathfrak{s o}(1,10)$ with g. In general, the rotations which give rise to supersymmetric reductions belong to the intersection of a spinor isotropy subalgebra of $\mathfrak{s o}(1,10)$ with $\mathfrak{g}$, but we choose to organise the results in terms of the spinor isotropy subalgebra, e.g., $\mathfrak{s u}(5)$ in this case.
} 
at the intersection of these hyperplanes, for example, $\theta_{4}=\theta_{5}$ and $\theta_{1}+\theta_{2}=0$, which corresponds to $\rho$ in an $\mathfrak{s p}(1) \times \mathfrak{s p}(1)$ subalgebra. The reduction now preserves a fraction $\nu=\frac{1}{4}$ of the supersymmetry.

Finally, in case (B) with $\rho_{\|}=0$ and $\rho_{\perp}$ fixing some directions, we can choose coordinates so that $\theta_{1}=0=\theta_{2}$. The supersymmetric locus consists of the intersection of the hyperplanes (3.17) with the hyperplanes $\theta_{1}=0$ and $\theta_{2}=0$. The resulting four hyperplanes are described by the equations

$$
\sum_{i=3}^{5} \mu_{i} \theta_{i}=0 \quad \text { where } \mu_{i}^{2}=1 .
$$

A generic $\rho$ in this locus is annihilated by four weights, hence the reduction preserves a fraction $\nu=\frac{1}{8}$ of the supersymmetry, and $\rho$ belongs to an $\mathfrak{s u}(3)$ subalgebra. We are free to specialise to any intersection of the above planes: simple intersections correspond to setting another one of the $\theta_{i}$ to zero, or equivalently to $\rho$ lying in an $\mathfrak{s u}(2)$ subalgebra. This enhances the supersymmetry to a fraction $\nu=\frac{1}{4}$. Finally, the only point in more than two hyperplanes is the origin, whence $\rho=0$ and hence the reduction preserves all the supersymmetry of the membrane, namely a fraction $\nu=\frac{1}{2}$. These results are summarised in Table 2.

As mentioned in the introduction, we could consider a discrete subgroup $\Gamma_{0} \subset \Gamma$ such that $\Gamma / \Gamma_{0}$ is compact. The corresponding eleven-dimensional configurations classified above, $\mathcal{M}_{\mathrm{M} 2} / \Gamma_{0}$, correspond to new smooth supersymmetric vacua which are asymptotic to $\mathbb{R}^{1,10} / \Gamma_{0}$. In the particular case in which $\xi=\tau_{\|}+\rho, \tau_{\|}$being an spacelike translation, $\mathcal{M}_{\mathrm{M} 2} / \Gamma_{0}$ corresponds to a stack of M2-branes in an eleven-dimensional fluxbrane vacua. We shall next concentrate on the Kaluza-Klein reductions.

\subsubsection{Explicit reductions}

We shall start by studying the reductions not involving timelike translations. The Killing vector can thus be written as $\xi=\partial_{z}+\lambda$, where $z$ stands for a longitudinal direction, i.e. $y^{2}$, and $\lambda$ stands for the infinitesimal rotation in the space transverse to the brane

$$
\begin{aligned}
\lambda & =\theta_{1}\left(y^{3} \partial_{4}-y^{4} \partial_{3}\right)+\theta_{2}\left(y^{5} \partial_{6}-y^{6} \partial_{5}\right) \\
& +\theta_{3}\left(y^{7} \partial_{8}-y^{8} \partial_{7}\right)+\theta_{4}\left(y^{9} \partial_{\natural}-y^{\natural} \partial_{9}\right) .
\end{aligned}
$$

The constant matrix $B$ introduced in (2.6) is an $8 \times 8$ matrix which can 


\begin{tabular}{|c|c|c|c|}
\hline Translation & Subalgebra & $\nu$ & $\operatorname{dim}$ \\
\hline \hline & $\mathfrak{s u}(4)$ & $\frac{1}{16}$ & 3 \\
& $\mathfrak{s u}(3)$ & $\frac{1}{8}$ & 2 \\
$a \partial_{1}$ & $\mathfrak{s p}(1) \times \mathfrak{s p}(1)$ & $\frac{1}{8}$ & 2 \\
$a \neq 0$ & $\mathfrak{s u}(2)$ & $\frac{1}{4}$ & 1 \\
& $\{0\}$ & $\frac{1}{2}$ & 0 \\
\hline$\partial_{+}$ & $\mathfrak{s u}(4)$ & $\frac{1}{16}$ & 2 \\
& $\mathfrak{s p}(1) \times \mathfrak{s p}(1)$ & $\frac{1}{8}$ & 1 \\
\hline \multirow{3}{*}{$a \partial_{0}$} & $\mathfrak{s u}(5)$ & $\frac{1}{32}$ & 4 \\
$0<|a|<\mu$ & $\mathfrak{s u}(2) \times \mathfrak{s u}(3)$ & $\frac{1}{16}$ & 3 \\
& $\mathfrak{s u}(4)$ & $\frac{1}{16}$ & 3 \\
& $\mathfrak{s p}(1) \times \mathfrak{s p}(1)$ & $\frac{1}{8}$ & 2 \\
\hline
\end{tabular}

Table 2: Supersymmetric reductions of the M2 brane. We indicate the form of the translation, the spinor isotropy subalgebra to which the rotation belongs, the fraction $\nu$ of the supersymmetry preserved and the dimension of the corresponding stratum of the moduli space $\mathcal{M}$ of supersymmetric reductions.

be written as

$$
B=\left(\begin{array}{cccccccc}
0 & -\theta_{1} & 0 & 0 & 0 & 0 & 0 & 0 \\
\theta_{1} & 0 & 0 & 0 & 0 & 0 & 0 & 0 \\
0 & 0 & 0 & -\theta_{2} & 0 & 0 & 0 & 0 \\
0 & 0 & \theta_{2} & 0 & 0 & 0 & 0 & 0 \\
0 & 0 & 0 & 0 & 0 & -\theta_{3} & 0 & 0 \\
0 & 0 & 0 & 0 & \theta_{3} & 0 & 0 & 0 \\
0 & 0 & 0 & 0 & 0 & 0 & 0 & -\theta_{4} \\
0 & 0 & 0 & 0 & 0 & 0 & \theta_{4} & 0
\end{array}\right)
$$

in the basis $\left\{x^{3}, x^{4}, \ldots x^{9}, x^{\natural}\right\}$ spanned by the adapted coordinates defined in (2.5). It is straightforward to derive the ten-dimensional metric

$$
\begin{aligned}
g=\tilde{\Lambda}^{1 / 2}\left\{V^{-1} d s^{2}\left(\mathbb{E}^{1,1}\right)\right. & \left.+d s^{2}\left(\mathbb{E}^{8}\right)\right\} \\
& -\tilde{\Lambda}^{-1 / 2} V\left(\theta_{1} \omega^{34}+\theta_{2} \omega^{56}+\theta_{3} \omega^{78}+\theta_{4} \omega^{9 \natural}\right)^{2},
\end{aligned}
$$


where we have introduced the notation $\omega^{i j}:=x^{i} d x^{j}-x^{j} d x^{i}$. The RR 1-form $A_{1}$, NS-NS 3 -form field strength $H_{3}$ and dilaton $\Phi$ are listed below:

$$
\begin{aligned}
A_{1} & =\tilde{\Lambda}^{-1} V\left(\theta_{1} \omega^{34}+\theta_{2} \omega^{56}+\theta_{3} \omega^{78}+\theta_{4} \omega^{9 \natural}\right) \\
H_{3} & =\operatorname{dvol} \mathbb{E}^{1,1} \wedge d V^{-1} \\
\Phi & =\frac{1}{2} \log \left(\tilde{\Lambda}^{3 / 2} \cdot V^{-1}\right),
\end{aligned}
$$

whereas the $\mathrm{RR}$ 4-form $H_{4}$ field strength vanishes. The configuration depends on an scalar function $\tilde{\Lambda}$ which is defined in terms of the scalar function $\Lambda$ appearing in Section 2.3, by

$$
\Lambda=V^{-2 / 3} \cdot \tilde{\Lambda},
$$

and equals

$$
\begin{aligned}
\tilde{\Lambda}=1+V\left\{( \theta _ { 1 } ) ^ { 2 } \left[\left(x^{3}\right)^{2}\right.\right. & \left.+\left(x^{4}\right)^{2}\right]+\left(\theta_{2}\right)^{2}\left[\left(x^{5}\right)^{2}+\left(x^{6}\right)^{2}\right] \\
& \left.+\left(\theta_{3}\right)^{2}\left[\left(x^{7}\right)^{2}+\left(x^{8}\right)^{2}\right]+\left(\theta_{4}\right)^{2}\left[\left(x^{9}\right)^{2}+\left(x^{\natural}\right)^{2}\right]\right\} .
\end{aligned}
$$

Notice that for arbitrary values of the angles $\theta_{i}$, the string coupling constant blows up, irrespectively of the direction, at large distances, whereas it is bounded from above by the constant $\left(\sum_{i}\left(\theta_{i}\right)^{2}\right)^{3 / 4} Q^{1 / 4}$ at $r \rightarrow 0$. Thus, it is always possible to have a weakly coupled region close to the origin, whereas as we move away from it, the M-theory description becomes more reliable.

As already discussed before, for arbitrary values of the deformation parameters $\left\{\theta_{i}\right\}$, the configuration would break supersymmetry completely, and its interpretation would be in terms of composites configurations involving fundamental strings lying in the $x^{1}$ direction at $r=0$ and, generically, four different F7-branes lying at $x^{3}=x^{4}=0, x^{5}=x^{6}=0, x^{7}=x^{8}=0$ and $x^{9}=x^{\natural}=0$, respectively. It is the presence of the F7-branes that breaks supersymmetry completely.

On the other hand, there are five different types of supersymmetric configurations

(1) If $\theta_{i}=0$ for all $i$, this is the standard type IIA configuration describing fundamental strings streching along the $x^{1}$ direction at $r=0$ and preserving $\nu=1 / 2$ of the spacetime supersymmetry.

(2) Setting $\theta_{3}=\theta_{4}=0$ and $\theta_{1}=\eta_{2} \theta_{2}$ corresponds to fundamental strings streching along the $x^{1}$ direction at $r=0$ and lying on an F5-brane that 


\begin{tabular}{|c|c|c|}
\hline$\nu$ & Object & Subalgebra \\
\hline \hline$\frac{1}{4}$ & $\mathrm{FA} \| \mathrm{F} 5$ & $\mathfrak{s u}(2)$ \\
$\frac{1}{8}$ & $\mathrm{FA} \| \mathrm{F} 3$ & $\mathfrak{s u}(3)$ \\
$\frac{1}{8}$ & $\mathrm{FA} \| \mathrm{F} 1$ & $\mathfrak{s p}(2)$ \\
$\frac{1}{16}$ & $\mathrm{FA} \| \mathrm{F} 1$ & $\mathfrak{s u}(4)$ \\
\hline
\end{tabular}

Table 3: Supersymmetric configurations of fundamental strings (FA) and fluxbranes

sits on $x^{3}=x^{4}=x^{5}=x^{6}=0$. This configuration preserves $\nu=1 / 4$ of the spacetime supersymmetry.

(3) Setting $\theta_{4}=0$ and $\theta_{1}=\eta_{2} \theta_{2}+\eta_{3} \theta_{3}$ corresponds to fundamental strings streching along the $x^{1}$ direction at $r=0$ and lying on an F3-brane that sits on $x^{3}=x^{4}=x^{5}=x^{6}=x^{7}=x^{8}=0$. This configuration preserves $\nu=1 / 8$ of the spacetime supersymmetry.

(4) Setting $\theta_{1}=\eta_{2} \theta_{2}$ and $\theta_{3}=\eta_{4} \theta_{4}$ corresponds to fundamental strings and $\frac{1}{4}$-BPS fluxstrings lying on $x^{1}$ at $r=0$. It preserves $\nu=1 / 8$.

(5) Setting $\theta_{1}=\eta_{2} \theta_{2}+\eta_{3} \theta_{3}+\eta_{4} \theta_{4}$, corresponds to fundamental strings and fluxstrings lying on $x^{1}$ at $r=0$. It preserves $\nu=1 / 16$.

The allowed supersymmetric configurations are summarised in Table 3.

If one sets the charge of the original M2-brane to zero, one recovers the corresponding fluxbrane configurations reviewed in 2.1. These do have some notion of flux associated with the integral over the transverse sections to the fluxbrane of $F_{2}=d A_{1}$, or wedge products of it. It is natural to compute this flux, when fundamental strings are switched on. We shall concentrate on F5-branes for simplicity. Notice that due to the presence of the fundamental strings, the RR 1-form potential depends on the point $\boldsymbol{x}=\left(x^{3}, x^{4}, x^{5}, x^{6}\right)$, but it is still invariant along the $x^{1}$ direction. Thus, the flux may depend on the point where we fix the transverse section along which we compute it. Fixing this point $\boldsymbol{x}$, and computing the integral

$$
\frac{1}{4 \pi} \int_{\mathbb{R}^{4}(\boldsymbol{x})} F_{2} \wedge F_{2}
$$

afterwards, one can check that the flux equals $\theta^{-2}$ (as in flat case) everywhere except at $\boldsymbol{x}=0$, where the string lies. In that point, the flux vanishes. This 
absence of flux at $\boldsymbol{x}=0$ seems to be consistent with the fact that there is no moduli associated with relative motions among fundamental strings and F5-branes. Indeed, a probe computation shows that fundamental strings do always feel a force when they sit away from $r=0$.

We shall next discuss the Kaluza-Klein reductions involving translations which are null. From our general discussion on freely-acting spacelike Killing vectors $\xi$, we already know the only allowed possibilities are those in which

$$
\xi=\partial_{+}+\lambda,
$$

where $\lambda$ is a rotation acting on the transverse space to the brane and without fixing any direction. It is thus the same as the one used previously, but this time no $\theta_{i}$ are allowed to vanish. By using our general formalism, the constant matrix $\mathrm{B}$ is again given by (3.18). This determines the tendimensional metric to be

$$
\begin{aligned}
g=\tilde{\Lambda}^{1 / 2} & \left(V^{-1 / 2}\left(d x^{2}\right)^{2}+V^{1 / 2} d s^{2}\left(\mathbb{E}^{8}\right)\right) \\
& -\tilde{\Lambda}^{-1 / 2} V^{1 / 2}\left(\theta_{1} \omega^{34}+\theta_{2} \omega^{56}+\theta_{3} \omega^{78}+\theta_{4} \omega^{9 \natural}+V^{-1} d x^{-}\right)^{2},
\end{aligned}
$$

where $\omega^{i j}:=x^{i} d x^{j}-x^{j} d x^{i}$. On the other hand, there are non-trivial RR 1-form $A_{1}$, NS-NS 3-form field strength $H_{3}$ and dilaton $\Phi$ which are listed below:

$$
\begin{aligned}
A_{1} & =\tilde{\Lambda}^{-1}\left\{\theta_{1} \omega^{34}+\theta_{2} \omega^{56}+\theta_{3} \omega^{78}+\theta_{4} \omega^{9 \natural}+V^{-1} d x^{-}\right\} \\
H_{3} & =d x^{-} \wedge d x^{2} \wedge d V^{-1} \\
\Phi & =\frac{3}{4} \log \left(\tilde{\Lambda} \cdot V^{1 / 3}\right) .
\end{aligned}
$$

The above type IIA configuration depends on an scalar function $\tilde{\Lambda}$ which was again defined in terms of the scalar function $\Lambda$ appearing in the general discussion section, by

$$
\Lambda=V^{1 / 3} \cdot \tilde{\Lambda}
$$

and equals

$$
\begin{aligned}
\tilde{\Lambda}=\left(\theta_{1}\right)^{2}\left[\left(x^{3}\right)^{2}+\left(x^{4}\right)^{2}\right] & +\left(\theta_{2}\right)^{2}\left[\left(x^{5}\right)^{2}+\left(x^{6}\right)^{2}\right] \\
& +\left(\theta_{3}\right)^{2}\left[\left(x^{7}\right)^{2}+\left(x^{8}\right)^{2}\right]+\left(\theta_{4}\right)^{2}\left[\left(x^{9}\right)^{2}+\left(x^{\natural}\right)^{2}\right] .
\end{aligned}
$$

As before, the string coupling constant blows up at large distances but is bounded from above by $\left(\sum_{i}\left(\theta_{i}\right)^{2}\right)^{3 / 4} Q^{1 / 4}$ at $r \rightarrow 0$. 
For arbitrary values of the deformation parameters $\theta_{i}\left(\theta_{i} \neq 0\right)$, the configuration would break supersymmetry completely. It is only when $\theta_{1}=$ $\eta_{2} \theta_{2}+\eta_{3} \theta_{3}+\eta_{4} \theta_{4}$ or $\theta_{1}=\eta_{2} \theta_{2}$ and $\theta_{3}=-\eta_{3} \eta_{4} \theta_{4}$, that the above configuration preserves $\nu=1 / 16$ or $\nu=1 / 8$, respectively. We do not have a physical interpretation for this set of configurations, and even though they were obtained by Kaluza-Klein reduction along the orbits of some Killing vectors which are spacelike everywhere, we were not able to prove whether these spacetimes have no causal singularities.

Let us finally move to the third possibility, the one involving timelike translations. The Killing vector is given by $\xi=a \partial_{0}+\lambda, \lambda$ standing for the spacetime rotation

$$
\begin{aligned}
\lambda & =\theta_{1}\left(x^{1} \partial_{2}-x^{2} \partial_{1}\right)+\theta_{2}\left(x^{3} \partial_{4}-x^{4} \partial_{3}\right) \\
& +\theta_{3}\left(x^{5} \partial_{6}-x^{6} \partial_{5}\right)+\theta_{4}\left(x^{7} \partial_{8}-x^{8} \partial_{7}\right)+\theta_{5}\left(x^{9} \partial_{\natural}-x^{\natural} \partial_{9}\right),
\end{aligned}
$$

where the timelike translation parameter is bound by $0<|a|<\mu$, with $\mu^{2}$ given by (3.13).

In this case, the constant matrix $B$ is a $10 \times 10$ matrix, which in the basis $\left\{x^{1}, x^{2}, \ldots x^{9}, x^{\natural}\right\}$ can be written as

$$
B=\left(\begin{array}{cccccccccc}
0 & -\theta_{1} & 0 & 0 & 0 & 0 & 0 & 0 & 0 & 0 \\
\theta_{1} & 0 & 0 & 0 & 0 & 0 & 0 & 0 & 0 & 0 \\
0 & 0 & 0 & -\theta_{2} & 0 & 0 & 0 & 0 & 0 & 0 \\
0 & 0 & \theta_{2} & 0 & 0 & 0 & 0 & 0 & 0 & 0 \\
0 & 0 & 0 & 0 & 0 & -\theta_{3} & 0 & 0 & 0 & 0 \\
0 & 0 & 0 & 0 & \theta_{3} & 0 & 0 & 0 & 0 & 0 \\
0 & 0 & 0 & 0 & 0 & 0 & 0 & -\theta_{4} & 0 & 0 \\
0 & 0 & 0 & 0 & 0 & 0 & \theta_{4} & 0 & 0 & 0 \\
0 & 0 & 0 & 0 & 0 & 0 & 0 & 0 & 0 & -\theta_{5} \\
0 & 0 & 0 & 0 & 0 & 0 & 0 & 0 & \theta_{5} & 0
\end{array}\right)
$$

The corresponding type IIA configurations have a ten-dimensional metric given by ${ }^{8}$

$$
\begin{aligned}
g=\tilde{\Lambda}^{1 / 2} & \left\{V^{-1} d s^{2}\left(\mathbb{E}^{2}\right)+d s^{2}\left(\mathbb{E}^{8}\right)\right\} \\
& -\tilde{\Lambda}^{-1 / 2} V^{-1}\left[\theta_{1} \omega^{12}+V\left\{\theta_{2} \omega^{34}+\theta_{3} \omega^{56}+\theta_{4} \omega^{78}+\theta_{5} \omega^{9 \natural}\right\}\right]^{2},
\end{aligned}
$$

where $\omega^{i \jmath}:=x^{i} d x^{j}-x^{\jmath} d x^{i}$. The RR 1-form $A_{1}$, NS-NS 3-form field strength

\footnotetext{
${ }^{8}$ We are grateful to Hannu Rajaniemi for spotting a small error in a previous version of this formula.
} 
$H_{3}$ and dilaton $\Phi$ are given by

$$
\begin{aligned}
A_{1} & =\tilde{\Lambda}^{-1}\left\{\theta_{1} \omega^{12}+V\left\{\theta_{2} \omega^{34}+\theta_{3} \omega^{56}+\theta_{4} \omega^{78}+\theta_{5} \omega^{9 \natural}\right\}\right\} \\
H_{3} & =a \mathrm{~d} \operatorname{vol} \mathbb{E}^{2} \wedge d V^{-1} \\
\Phi & =\frac{1}{2} \log \left(\tilde{\Lambda}^{3 / 2} \cdot V^{-1}\right) .
\end{aligned}
$$

The configuration depends on an scalar function $\tilde{\Lambda}$ which is defined in terms of the scalar function $\Lambda$ appearing in the general discussion section, by

$$
\Lambda=V^{-2 / 3} \cdot \tilde{\Lambda},
$$

and equals

$$
\begin{aligned}
\tilde{\Lambda}=-a^{2}+ & \left(\theta_{1}\right)^{2}\left[\left(x^{1}\right)^{2}+\left(x^{2}\right)^{2}\right]+V\left\{\left(\theta_{2}\right)^{2}\left[\left(x^{3}\right)^{2}+\left(x^{4}\right)^{2}\right]\right. \\
& \left.+\left(\theta_{3}\right)^{2}\left[\left(x^{5}\right)^{2}+\left(x^{6}\right)^{2}\right]+\left(\theta_{4}\right)^{2}\left[\left(x^{7}\right)^{2}+\left(x^{8}\right)^{2}\right]+\left(\theta_{5}\right)^{2}\left[\left(x^{9}\right)^{2}+\left(x^{\natural}\right)^{2}\right]\right\} .
\end{aligned}
$$

For generic values of the five parameters $\left\{\theta_{i}\right\}, i=1,2, \ldots, 5$, the above configuration breaks supersymmetry. There are several loci in this fivedimensional parameter space where supersymmetry is restored. If the rotation along the $\mathrm{M} 2$-brane is non-vanishing, $\theta_{1} \neq 0$, there are two possibilities to be discussed:

(1) $\sum_{i} \mu_{i} \theta_{i}=0$ such that $\mu_{i}^{2}=1$. The full rotation belongs to the $\mathfrak{s u}(5)$ spinor isotropy subalgebra. This configuration preserves $\nu=1 / 32$ of the spacetime supersymmetry.

(2) If the rotation belongs to the $\mathfrak{s u}(2) \times \mathfrak{s u}(3)$ spinor isotropy subalgebra, there are two subcases to be considered due to the isometries of the starting M2-brane configuration. Indeed,

(2.1) If the rotation on the brane $\left(\theta_{1}\right)$ belongs to the $\mathfrak{s u}(2)$ subalgebra. In this case, $\mu_{1} \theta_{1}+\mu_{2} \theta_{2}=0$ and $\sum_{i=3,4,5} \mu_{i} \theta_{i}=0$.

(2.2) If the rotation on the brane $\left(\theta_{1}\right)$ belongs to the $\mathfrak{s u}(3)$ subalgebra. In this case, $\mu_{4} \theta_{4}+\mu_{5} \theta_{5}=0$ and $\sum_{i=1,2,3} \mu_{i} \theta_{i}=0$.

Notice that all other possibilities are conjugate to the ones selected above. Both of them preserve $\nu=1 / 16$.

On the other hand, if $\theta_{1}=0$, one is just left with a transverse rotation $\lambda=\rho_{\perp}$. Due to the isometries of the background configuration, we are just left to consider two possibilities: 
(1) If $\rho_{\perp}$ belongs to the $\mathfrak{s u}(4)$ spinor isotropy subalgebra. In that case, $\sum_{i=2, \ldots, 5} \mu_{i} \theta_{i}=0$. The resulting configuration preserves $\nu=1 / 16$.

(2) If $\rho_{\perp}$ belongs to the $\mathfrak{s p}(1) \times \mathfrak{s p}(1)$ subalgebra. In that case, $\mu_{2} \theta_{2}+\mu_{3} \theta_{3}=$ 0 and $\mu_{4} \theta_{4}+\mu_{5} \theta_{5}=0$. The resulting configuration preserves $\nu=1 / 8$.

As in the former family of reductions, the physical interpretation and the causal structure of the above spacetimes is still missing.

\subsection{Supersymmetric reductions of the delocalised M2-brane}

The standard Kaluza-Klein reduction of the M2-brane to obtain the D2brane, requires that the M2-brane be delocalised along one transverse direction; that is, that the M2-brane admit a Killing vector which is a translation along a transverse direction. The metric of the spacetimes exterior to such a membrane is again of the general form (1.7) but now with three factors:

$$
g=V^{-2 / 3} d s^{2}\left(\mathbb{E}^{1,2}\right)+V^{1 / 3} d z^{2}+V^{1 / 3} d s^{2}\left(\mathbb{E}^{7}\right),
$$

where $z$ is the transverse coordinate along which the membrane is delocalised and $V=1+|Q| / r^{5}$ is a harmonic function on $\mathbb{E}^{7}$ depending only on the radial distance. The symmetry group is now

$$
G=\operatorname{ISO}(1,2) \times \mathbb{R} \times \operatorname{SO}(7)
$$

with Lie algebra

$$
\mathfrak{g}=\left(\mathbb{R}^{1,2} \rtimes \mathfrak{s o}(1,2)\right) \times \mathbb{R} \times \mathfrak{s o}(7) .
$$

Therefore a Killing vector may be decomposed as

$$
\xi=\tau_{\|}+\tau_{\perp}+\lambda_{\|}+\rho_{\perp}
$$

with the same notation as above.

\subsubsection{Freely-acting spacelike isometries}

We proceed as before by using the freedom of acting by $G$ in order to bring $\lambda$ to a normal form. Either $\lambda=0$ or else it can be brought into one of three normal forms: an infinitesimal boost, rotation or null rotation. We can again discard the boost since this leads to a $\xi$ which is not spacelike. The same reason forces $\tau_{\|}=0$ in the case where $\lambda$ is a null rotation. Arguing as in the previous section, we are left with the following two cases of freely-acting spacelike Killing vectors: 
(A) $\xi=\tau_{\perp}+\nu_{\|}+\rho_{\perp}$, where $\tau \neq 0$ for otherwise $\xi$ does not act freely;

(B) $\xi=\tau_{\|}+\tau_{\perp}+\rho_{\|}+\rho_{\perp}$, where $\tau_{\|}+\tau_{\perp}$ is spacelike, but where

(i) if $\rho_{\|}=0$ then $\tau_{\|}$can be either spacelike, timelike or null; and

(ii) if $\rho_{\|} \neq 0$, then $\tau_{\|}$is timelike.

In all cases the decomposition of $\xi$ is orthogonal relative to the brane metric. We also remind the reader that $\tau, \rho$ and $\nu$ stand, respectively, for a translation, a rotation and a null rotation, and that the subscripts $\|$ and $\perp$ denote directions tangent to and perpendicular to the brane, respectively. Notice that $\rho_{\perp}$ here always fixes at least one direction since it defines a tangent vector field on an even-dimensional sphere.

\subsubsection{Moduli space of smooth reductions}

We now describe the different strata of the moduli space of smooth reductions. In case $(\mathrm{A})$, coordinates can be chosen so that the Killing vector $\xi$ takes the form

$$
\xi=a \partial_{z}+N_{+2}+\theta_{2} R_{34}+\theta_{3} R_{56}+\theta_{4} R_{78} .
$$

The moduli space is obtained by projectivising and quotienting by the action of the Weyl group and hence this stratum of the moduli space is threedimensional. Supersymmetry will then select a two-dimensional locus.

In case (B) with $\rho_{\|} \neq 0, \tau_{\|}$must be timelike, whence

$$
\xi=a \partial_{0}+b \partial_{9}+\theta_{1} R_{12}+\theta_{2} R_{34}+\theta_{3} R_{56}+\theta_{4} R_{78},
$$

where $|a|<|b|$. There are six free parameters, whence this stratum is fivedimensional after projectivisation. Supersymmetry will then select a fourdimensional locus.

Finally in case (B) with $\rho_{\|}=0$, we have to distinguish between three cases depending on whether $\tau_{\|}$is timelike, spacelike or null. If $\tau_{\|}$is timelike we can bring $\xi$ to the form

$$
\xi=a \partial_{0}+b \partial_{9}+\theta_{2} R_{34}+\theta_{3} R_{56}+\theta_{4} R_{78}
$$

with $|a|<|b|$. As a result there are five free parameters yielding a fourdimensional moduli space. Supersymmetry will further select a three-dimensional locus. Similarly if $\tau_{\|}$is spacelike, we can bring $\xi$ to the form

$$
\xi=a \partial_{1}+b \partial_{9}+\theta_{2} R_{34}+\theta_{3} R_{56}+\theta_{4} R_{78},
$$


where now $a$ and $b$ cannot both be zero. Again we have a four-dimensional moduli space of smooth reductions with a codimension-one locus of supersymmetric reductions. Finally if $\tau_{\|}$is null, $\xi$ takes the form

$$
\xi=\partial_{+}+b \partial_{9}+\theta_{2} R_{34}+\theta_{3} R_{56}+\theta_{4} R_{78},
$$

with $b \neq 0$. This gives rise to a three-dimensional moduli space of smooth reductions with a two-dimensional locus of supersymmetric reductions.

\subsubsection{Absence of closed causal curves}

The purpose of the present subsection is to analytically prove that despite the intuition, the above spacetimes do not have closed causal curves. We shall concentrate on spacetimes reduced along the orbits infinitesimally generated by Killing vectors $\xi$ which act non-trivially either on a timelike or a lightlike direction. For simplicity, we will not allow the transverse rotation parameters to be arbitrary, but set all of them to zero.

Let us start by analysing the problem of existence of closed causal curves in an M2-brane background delocalised in one transverse direction $z$ reduced along the orbits of the Killing vector

$$
\xi=a \partial_{0}+b \partial_{z} .
$$

The only condition that such a Killing vector is required to satisfy is to be spacelike everywhere. This requirement provides us with the constraint that, for all $r$,

$$
\|\xi\|^{2}=V^{-2 / 3}(r)\left(-a^{2}+V(r) b^{2}\right)>0
$$

To analyse this question, it is convenient to change coordinates to an adapted coordinate system, in which the Killing vector becomes a single spacelike translation $\xi=\partial_{z^{\prime}}$. In this case, this is easily achieved by a linear transformation in the original $\left\{x^{0}, z\right\}$ space. In the new coordinate system $\left\{t^{\prime}, z^{\prime}\right\}$, the eleven-dimensional takes the form

$$
\begin{aligned}
g=-V^{-2 / 3}\left[a^{2}\left(d z^{\prime}\right)^{2}+b^{-2}\left(d t^{\prime}\right)^{2}+2 a b^{-1} d z^{\prime} d t^{\prime}\right]+b^{2} V^{1 / 3}\left(d z^{\prime}\right)^{2} & \\
& +V^{1 / 3} d s^{2}\left(\mathbb{E}^{7}\right) .
\end{aligned}
$$

What we would like to know is whether there exist closed causal curves $x(\lambda)$, i.e., $\left\|\frac{d x}{d \lambda}\right\|^{2} \leq 0$ joining the points $\left(t_{0}^{\prime}, x_{0}^{i}, z_{0}^{\prime}\right)$ and $\left(t_{0}^{\prime}, x_{0}^{i}, z_{0}^{\prime}+\Delta\right)$, since they become identified in the quotient. Let us assume that such a curve 
exists. If so, there must exist at least one value $\lambda^{*}$ of the affine parameter $\lambda$ where the timelike component of the tangent vector to the curve vanishes:

$$
\exists \lambda^{\star} \text { such that }\left.\frac{d t^{\prime}}{d \lambda}\right|_{\lambda^{*}}=0 .
$$

If one computes the norm of such a tangent vector at $\lambda^{*}$, one derives the inequality

$$
\left.\|\xi\|^{2}\left(\lambda^{*}\right) \frac{d z^{\prime}}{d \lambda}\right|_{\lambda^{*}} ^{2}+\left.V^{1 / 3} \sum_{i} \frac{d x^{i}}{d \lambda}\right|_{\lambda^{*}} ^{2} \leq 0 .
$$

Due to the constraint (3.24), it is clear that the left hand side of the above norm is the sum of positively defined terms, so that the inequality can never be satisfied. This already shows the non-existence of closed timelike curves. Furthermore, the only possibility for the equality to be satisfied is whenever, for all $i$,

$$
\left.\frac{d z^{\prime}}{d \lambda}\right|_{\lambda^{*}}=\left.\frac{d x^{i}}{d \lambda}\right|_{\lambda^{*}}=0
$$

But the existence of one point where the tangent vector to the causal curve vanishes identically violates the fact that $\lambda$ is an affine parameter. We thus conclude that no closed lightlike curves are allowed in this spacetime.

The corresponding proof for the action generated by $\xi=\partial_{+}+b \partial_{z}$ involves similar ideas and techniques. In this case, the requirement of having an everywhere spacelike Killing vector gives rise to the condition

$$
V(r)^{1 / 3} b^{2}>0 \Rightarrow|b|>0 .
$$

By a linear transformation, we can move to an adapted coordinate system $\left\{z^{\prime}, x^{+\prime}\right\}$ in which the Killing vector becomes a single translation $\xi=\partial_{z^{\prime}}$ and the eleven-dimensional metric takes the form

$$
\begin{aligned}
g=2 V^{-2 / 3} d x^{-}\left(d z^{\prime}+b^{-1} d x^{+\prime}\right)+V^{1 / 3} b^{2} & \left(d z^{\prime}\right)^{2} \\
& +V^{1 / 3} d s^{2}\left(\mathbb{E}^{7}\right)+V^{-2 / 3}\left(d x^{2}\right)^{2} .
\end{aligned}
$$

If we again assume the existence of a closed causal curve of affine parameter $\lambda$ joining the points $\left(x_{0}^{+\prime}, x_{0}^{-}, x_{0}^{i}, z_{0}^{\prime}\right)$ and $\left(x_{0}^{+\prime}, x_{0}^{-}, x_{0}^{i}, z_{0}^{\prime}+\Delta\right)$, there must necessarily exist at least one value for this affine parameter $\lambda^{*}$ where

$$
\exists \lambda^{*} \text { such that }\left.\frac{d x^{-}}{d \lambda}\right|_{\lambda^{*}}=0 .
$$

By computing the norm of the tangent vector to the causal curve at the point $\lambda^{*}$, and using the fact that $|b|>0$, it is immediate to show the non-existence of such closed causal curves by the same argument used before. 


\subsubsection{Supersymmetry}

Now we determine the locus of supersymmetric reductions. In all the cases of smooth reductions, the rotation component of the Killing vector takes the general form

$$
\rho=\theta_{1} R_{12}+\theta_{2} R_{34}+\theta_{3} R_{56}+\theta_{4} R_{78},
$$

which is the special case of $(3.16)$ corresponding to $\theta_{5}=0$. This allows us to reduce the determination of the supersymmetric locus to the case of the M2-brane, with the added feature that when in addition $\theta_{1}=0$ we have the option of adding a null rotation to $\xi$ with the effect of halving the fraction of supersymmetry, as described in Section 2.2. We will not repeat the arguments here and simply state the results, which are illustrated in Table 4.

Before moving into the explicit Kaluza-Klein reductions, let us stress that the previous classification gives rise to a wealth of smooth supersymmetric M-theory backgrounds, $\mathcal{M}_{\mathrm{M} 2} / \Gamma_{0}$ by considering discrete subgroups $\Gamma_{0} \subset$ $\Gamma$. These include a stack of delocalised M2-branes and eleven-dimensional fluxbranes $\left(\xi=\partial_{1}+\rho_{\perp}\right)$ or eleven-dimensional nullbranes $\left(\xi=\partial_{1}+\nu_{\|}\right)$. The latter is an example of an eleven-dimensional time-dependent background in which a compact spacelike worldvolume dimension shrinks as time evolves down to a minimum size and then re-expands. It would be very interesting to understand the physics on the throat of the brane in such an scenario.

\subsubsection{Explicit reductions}

In the following, we shall explicitly write down the different type IIA configurations obtained by the inequivalent Kaluza-Klein reductions identified and classified in previous subsections. Let us start by the subspace of the moduli space in which the parameter $a$ associated with the spacelike translation $a \partial_{1}$ is set to zero. In order to discuss both null and flux branes at the same time, we shall present the Kaluza-Klein reduction along the orbits of the Killing vector $\xi=\partial_{z}+\lambda$, where the infinitesimal transformation $\lambda$ is given by

$$
\lambda=\beta B_{02}+\theta_{1} R_{12}+\theta_{2} R_{34}+\theta_{3} R_{56}+\theta_{4} R_{78} .
$$

Thus, whenever $\beta=0$, we will be discussing composite configurations of D2branes and flux branes; whenever $|\beta|=\left|\theta_{1}\right|$, we will be discussing composite configurations of D2-branes, null branes and flux branes.

The constant matrix $B$ is a $9 \times 9$ matrix which does not act both on the $x^{9}$ and $z$ directions. Relative to the basis $\left\{x^{0}, x^{1}, \ldots, x^{8}\right\}$, it is given 


\begin{tabular}{|c|c|c|c|}
\hline Translation & Subalgebra & $\nu$ & $\operatorname{dim}$ \\
\hline \hline$a \partial_{1}+b \partial_{z}$ & $\mathfrak{s u}(3)$ & $\frac{1}{8}\left(\frac{1}{16}\right)$ & $3(2)$ \\
$a, b$ not both 0 & $\mathfrak{s u}(2)$ & $\frac{1}{4}\left(\frac{1}{8}\right)$ & $2(1)$ \\
$(a=0, b \neq 0)$ & $\{0\}$ & $\frac{1}{2}\left(\frac{1}{4}\right)$ & $1(0)$ \\
\hline$\partial_{+}+b \partial_{z}$ & $\mathfrak{s u}(3)$ & $\frac{1}{8}$ & 2 \\
$b \neq 0$ & $\mathfrak{s u}(2)$ & $\frac{1}{4}$ & 1 \\
& $\{0\}$ & $\frac{1}{2}$ & 0 \\
\hline & $\mathfrak{s u}(4)$ & $\frac{1}{16}$ & 4 \\
$a \partial_{0}+b \partial_{z}$ & $\mathfrak{s u}(3)$ & $\frac{1}{8}$ & 3 \\
& $\mathfrak{s p}(1) \times \mathfrak{s p}(1)$ & $\frac{1}{8}$ & 3 \\
$|b|>|a|>0$ & $\mathfrak{s u}(2)$ & $\frac{1}{4}$ & 2 \\
& $\{0\}$ & $\frac{1}{2}$ & 1 \\
\hline
\end{tabular}

Table 4: Supersymmetric reductions of the delocalised M2-brane. We indicate the form of the translation, the spinor isotropy subalgebra to which the rotation belongs, the fraction $\nu$ of the supersymmetry preserved and the dimension of the corresponding stratum of the moduli space $\mathcal{M}$ of supersymmetric reductions. The numbers in parentheses indicate the values in the presence of a null rotation, which can only occur when the translation is trasverse.

explicitly by

$$
B=\left(\begin{array}{ccccccccc}
0 & 0 & \beta & 0 & 0 & 0 & 0 & 0 & 0 \\
0 & 0 & -\theta_{1} & 0 & 0 & 0 & 0 & 0 & 0 \\
\beta & \theta_{1} & 0 & 0 & 0 & 0 & 0 & 0 & 0 \\
0 & 0 & 0 & 0 & -\theta_{2} & 0 & 0 & 0 & 0 \\
0 & 0 & 0 & \theta_{2} & 0 & 0 & 0 & 0 & 0 \\
0 & 0 & 0 & 0 & 0 & 0 & -\theta_{3} & 0 & 0 \\
0 & 0 & 0 & 0 & 0 & \theta_{3} & 0 & 0 & 0 \\
0 & 0 & 0 & 0 & 0 & 0 & 0 & 0 & -\theta_{4} \\
0 & 0 & 0 & 0 & 0 & 0 & 0 & \theta_{4} & 0
\end{array}\right) .
$$

The corresponding type IIA configurations have a ten-dimensional metric given by

$$
g=\tilde{\Lambda}^{1 / 2}\left\{V^{-1 / 2} d s^{2}\left(\mathbb{E}^{1,2}\right)+V^{1 / 2} d s^{2}\left(\mathbb{E}^{7}\right)\right\}
$$




$$
-\tilde{\Lambda}^{-1 / 2} V^{1 / 2}\left\{V^{-1}\left[\beta \omega^{02}+\theta_{1} \omega^{12}\right]+\theta_{2} \omega^{34}+\theta_{3} \omega^{56}+\theta_{4} \omega^{78}\right\}^{2},
$$

where again $\omega^{i j}=x^{i} d x^{j}-x^{j} d x^{i}$. In addition, the RR 1-form $A_{1}$, NS-NS 3 -form field strength $H_{3}$, RR 4-form $H_{4}$ and dilaton $\Phi$ are given by

$$
\begin{aligned}
A_{1} & =\tilde{\Lambda}^{-1}\left\{V^{-1}\left[\beta \omega^{02}+\theta_{1} \omega^{12}\right]+\theta_{2} \omega^{34}+\theta_{3} \omega^{56}+\theta_{4} \omega^{78}\right\} \\
H_{3} & =-\left(\beta x^{2} d x^{1} \wedge d x^{2}+\theta_{1} x^{2} d x^{0} \wedge d x^{2}+\left(\beta x^{0}+\theta_{1} x^{1}\right) d x^{0} \wedge d x^{1}\right) \wedge d V^{-1} \\
H_{4} & =\operatorname{dvol}\left(\mathbb{E}^{1,2}\right) \wedge d V^{-1} \\
\Phi & =\frac{3}{4} \log \left(\tilde{\Lambda} \cdot V^{1 / 3}\right) .
\end{aligned}
$$

The configuration depends on an scalar function $\tilde{\Lambda}$ which is defined in terms of the scalar function $\Lambda$ appearing in the general discussion section, by

$$
\Lambda=V^{1 / 3} \cdot \tilde{\Lambda}
$$

and equals

$$
\begin{aligned}
\tilde{\Lambda}=1+V^{-1}\left\{\left(x^{2}\right)^{2}\left[\theta_{1}^{2}-\beta^{2}\right]+\left(\beta x^{0}\right.\right. & \left.\left.+\theta_{1} x^{1}\right)^{2}\right\}+\left(\theta_{2}\right)^{2}\left[\left(x^{3}\right)^{2}+\left(x^{4}\right)^{2}\right] \\
& +\left(\theta_{3}\right)^{2}\left[\left(x^{5}\right)^{2}+\left(x^{6}\right)^{2}\right]+\left(\theta_{4}\right)^{2}\left[\left(x^{7}\right)^{2}+\left(x^{8}\right)^{2}\right] .
\end{aligned}
$$

Whenever $\left|\theta_{1}\right|<|\beta|$, there always exists a Lorentz transformation such that $\theta_{1} R_{12}+\beta B_{02}$ becomes a pure boost. Therefore, such a configuration always breaks supersymmetry. Furthermore, the corresponding Killing vector is no longer spacelike everywhere, pointing out to the existence of regions in spacetime where there exist closed timelike curves. These configurations would correspond, whenever we restrict ourselves to regions of spacetime with no causal sickness, to similar cosmological scenarios to the ones discussed in $[45,46,48,49]$, but this time taking place on the worldvolume of a $2+1$ brane. By switching on the moduli associated with transverse rotations, one is just adding F7-branes into the discussion. On the other hand, if $\left|\theta_{1}\right|>|\beta|$, there always exists a Lorentz transformation mapping $\theta_{1} R_{12}+\beta B_{02}$ to a pure rotation, whose physical interpretation has already been given.

Let us concentrate on the interpretation of the different supersymmetric loci summarised in 4 . If we set $\beta=0$, there are four different possibilities to be considered:

(1) The case $\theta_{i}=0$ for all $i$ corresponds to the well-known D2-brane in type IIA preserving $\nu=1 / 2$ of the spacetime supersymmetry.

(2) We must distinguish between two case of two non-vanishing $\theta \mathrm{s}$ :

(i) If $\theta_{1}=\eta_{2} \theta_{2}$, the configuration describes a D2-brane in the (12)plane and an F5-brane along the (56789)-plane sitting at $x^{1}=$ $x^{2}=x^{3}=x^{4}=0$. It preserves $\nu=1 / 4$ of the spacetime supersymmetry, with Killing spinors being preserved by an $\mathfrak{s u}(2)$ subalgebra. 
(ii) If $\theta_{2}=\eta_{3} \theta_{3}$, the configuration describes a $\mathrm{D} 2$-brane in the (12)plane and an F5-brane along the (12789)-plane sitting at $x^{3}=$ $x^{4}=x^{5}=x^{6}=0$. It preserves $\nu=1 / 4$ of the spacetime supersymmetry, with Killing spinors again preserved by an $\mathfrak{s u}(2)$ subalgebra.

(3) There are again two distinct case of three non-vanishing $\theta \mathrm{s}$ :

(i) If $\theta_{1}=\eta_{2} \theta_{2}+\eta_{3} \theta_{3}$, the configuration describes a D2-brane in the (12)-plane and an F3-brane along the (789)-plane sitting at $x^{1}=x^{2}=x^{3}=x^{4}=x^{5}=x^{6}=0$. It preserves $\nu=1 / 8$ of the spacetime supersymmetry, with Killing spinors being preserved by an $\mathfrak{s u}(3)$ subalgebra.

(ii) If $\theta_{2}=\eta_{3} \theta_{3}+\eta_{4} \theta_{4}$, the F3-brane extends along the (129)-plane sitting at $x^{3}=x^{4}=x^{5}=x^{6}=x^{7}=x^{8}=0$. It preserves the same amount of supersymmetry as the previous case due to the existence of an $\mathfrak{s u}(3)$ subalgebra preserving some Killing spinors.

(4) As explained in the general discussion about preservation of supersymmetry, there are two inequivalent ways of preserving Killing spinors when four of the $\theta$ s are non-vanishing:

(i) $\theta_{1}=\eta_{2} \theta_{2}+\eta_{3} \theta_{3}+\eta_{4} \theta_{4}$. This configuration describes a D2-brane in the (12)-plane and a flux string along the $x^{9}$ direction, preserving $\nu=1 / 16$ of the spacetime supersymmetry. This is the one associated with the $\mathfrak{s u}(4)$ isotropy algebra discussed before.

(ii) $\theta_{1}=\eta_{2} \theta_{2}$ and $\theta_{3}=\eta_{4} \theta_{4}$. This second possibility involves a D2-brane and a maximally supersymmetric flux string in the $x^{9}$ direction. It preserves $\nu=1 / 8$ and it is associated with the $\mathfrak{s p}(1) \times \mathfrak{s p}(1)$ isotropy algebra.

If $\beta \neq 0$, the only allowed possibility preserving supersymmetry requires $\left|\theta_{1}\right|=|\beta|$. Let us thus concentrate on this case. Depending on whether the remaining parameters vanish or satisfy certain linear relations, we distinguish between the following configurations

(1) If $\theta_{i}=0 i=2,3,4$, it describes a D2-brane in the (12)-plane and a null brane. This composite configuration preserves $\nu=1 / 4$ of the spacetime supersymmetry.

(2) If one of the $\theta \mathrm{s}$ is non-vanishing, the corresponding configuration breaks supersymmetry completely, due to the presence of a F7-brane besides the previous D2-brane and null brane. 
(3) If two of the $\theta$ s are non-vanishing, we shall distinguish among two cases:

(i) If $\theta_{2} \neq \eta_{3} \theta_{3}$, the configuration describes a system of two intersecting F7-branes (besides the composite system of a D2-brane and a null brane), such that all supersymmetry is broken.

(ii) If $\theta_{2}=\eta_{3} \theta_{3}$, the intersection among the F7-branes gives rise to the so-called F5-brane, extending along the (12789)-plane and sitting at $x^{3}=x^{4}=x^{5}=x^{6}=0$. The full configuration includes the previous pair D2/null-brane system, thus preserving $\nu=1 / 8$.

(4) If all $\theta$ s are non-vanishing, we need to distinguish between two cases:

(i) If $\theta_{2} \neq \eta_{3} \theta_{3}+\eta_{4} \theta_{4}$, the configuration describes the intersection of three intersecting F7-branes, besides the D2-null-brane system, thus breaking all supersymmetry.

(ii) If $\theta_{2}=\eta_{3} \theta_{3}+\eta_{4} \theta_{4}$, the intersection of the F7-branes gives rise to a F3-brane extending along the (129)-plane and sitting at $x^{k}=0$ $\mathrm{k}=3, \ldots, 8$. Thus, there exists a composite configuration involving a D2-brane, null brane and F3-brane preserving $\nu=1 / 16$.

The supersymmetric configurations discussed above are summarised in table 5 .

\begin{tabular}{|c|c|l|}
\hline$\nu$ & Object & \multicolumn{1}{|c|}{ Subalgebra } \\
\hline \hline$\frac{1}{4}$ & $\mathrm{D} 2 \perp \mathrm{F} 5$ & $\mathfrak{s u}(2)$ \\
& $\mathrm{D} 2 \| \mathrm{F} 5$ & \\
$\frac{1}{4}$ & $\mathrm{D} 2+\mathrm{N}$ & $\mathbb{R}$ \\
$\frac{1}{8}$ & $\mathrm{D} 2 \perp \mathrm{F} 3$ & $\mathfrak{s u}(3)$ \\
& $\mathrm{D} 2 \| \mathrm{F} 3$ & \\
$\frac{1}{8}$ & $\mathrm{D} 2+\mathrm{N}+\mathrm{F} 5$ & $\mathfrak{s u}(2) \times \mathbb{R}$ \\
$\frac{1}{16}$ & $\mathrm{D} 2 \perp \mathrm{F} 1$ & $\mathfrak{s u}(4)$ \\
$\frac{1}{16}$ & $\mathrm{D} 2 \perp \mathrm{F} 1$ & $\mathfrak{s p}(1) \times \mathfrak{s p}(1)$ \\
$\frac{1}{16}$ & $\mathrm{D} 2+\mathrm{N}+\mathrm{F} 3$ & $\mathfrak{s u}(3) \times \mathbb{R}$ \\
\hline
\end{tabular}

Table 5: Supersymmetric configurations of D2-branes (D2), fluxbranes and nullbranes.

It is interesting to compute the fluxes associated with fluxbranes in the presence of D2-branes. We shall concentrate on the F5-brane, for simplicity. 
According to table 5 , there are two different cases to be discussed. Let us start with $D 2 \| F 5$. This corresponds to setting $\theta_{1}=\theta_{4}=\beta=0$ and $\theta \equiv \theta_{2}=\theta_{3}$ in (3.26) and (3.27). Notice, by inspection of (3.27), the absence of NS-NS charge in this case and the fact that the RR 1-form does not depend on the point of the F5-brane worldvolume, as it was the case for the fundamental strings analysed in the previous section. It is clear then that the flux "carried" by the F5-brane is exactly the same as in flat space

$$
\frac{1}{8 \pi^{2}} \int_{\mathbb{R}^{4}} F_{2} \wedge F_{2}=\theta^{-2}
$$

It is interesting to note that the solution we found is not expected to be the most general one for this system, due to the existence of moduli. Indeed, if one probes the F5-brane background with a D2-brane oriented as described above, the D2-brane does not feel any force for an arbitrary transverse distance among both objects, whereas in the solution described here the D2-brane lies on the F5-brane. This is certainly not necessary, as it was for fundamental strings.

When the D2-branes are transverse to the F5-brane, the RR 1-form does depend on the radial distance along the (56789)-plane spanned by the F5brane, whereas there appears some NS-NS charge in the radial direction on the (12)-plane, where the D2-brane lies. Nevertheless, proceeding as for the fundamental strings, that is, choosing a point on the F5-brane and keeping it as a parameter, it can be shown that

$$
F_{2} \wedge F_{2} \propto \frac{\partial\left(r_{2} \cdot \tilde{\Lambda}^{-2}\right)}{\partial r_{1}} d r_{1} \wedge d \varphi_{1} \wedge d r_{2} \wedge d \varphi_{1}
$$

where $x^{1}+i x^{2}=r_{1} e^{i \varphi_{1}}$ and $x^{3}+i x^{4}=r_{1} e^{i \varphi_{2}}$. Therefore, the flux equals the one of the flat spacetime, except at the origin where it vanishes.

Let us move to the region of the moduli space where the extra spacelike translation parameter is non-vanishing. In other words, let us consider the Kaluza-Klein reduction along the orbits of the Killing vector $\xi=\partial_{z}+\alpha$, where

$$
\alpha=a \partial_{2}+\theta_{2} R_{34}+\theta_{3} R_{56}+\theta_{4} R_{78}
$$

The constant matrix $B$ is a $7 \times 7$ matrix which does not act on the $\left\{x^{0}, x^{1}, x^{9}\right\}$ 
directions. It is given explicitly, in the basis $\left\{x^{2}, \ldots, x^{8}\right\}$, by

$$
B=\left(\begin{array}{ccccccc}
0 & 0 & 0 & 0 & 0 & 0 & 0 \\
0 & 0 & -\theta_{2} & 0 & 0 & 0 & 0 \\
0 & \theta_{2} & 0 & 0 & 0 & 0 & 0 \\
0 & 0 & 0 & 0 & -\theta_{3} & 0 & 0 \\
0 & 0 & 0 & \theta_{3} & 0 & 0 & 0 \\
0 & 0 & 0 & 0 & 0 & 0 & -\theta_{4} \\
0 & 0 & 0 & 0 & 0 & \theta_{4} & 0
\end{array}\right)
$$

Since $\alpha$ involves a translation, the constant vector $C$ defined in (2.6) taking care of the inhomogeneous part of the infinitesimal transformation is nonvanishing. It is a given by a 7 -vector

$$
(\boldsymbol{C})^{t}=(a, \overrightarrow{0}) .
$$

The corresponding type IIA configurations have a ten-dimensional metric given by

$$
\begin{aligned}
g=\tilde{\Lambda}^{1 / 2}\left\{V^{-1 / 2}(\right. & \left.\left.d s^{2}\left(\mathbb{E}^{1,1}\right)+\left(d x^{2}\right)^{2}\right)+V^{1 / 2}\left(d s^{2}\left(\mathbb{E}^{6}\right)+\left(d x^{9}\right)^{2}\right)\right\} \\
& -\tilde{\Lambda}^{-1 / 2} V^{1 / 2}\left\{a V^{-1} d x^{2}+\theta_{2} \omega^{34}+\theta_{3} \omega^{56}+\theta_{4} \omega^{78}\right\}^{2},
\end{aligned}
$$

where we are still using the notation $\omega^{i j}=x^{i} d x^{j}-x^{j} d x^{i}$. In addition, the RR 1-form $A_{1}$, NS-NS 3-form field strength $H_{3}$, RR 4-form $H_{4}$ and dilaton $\Phi$ are listed below:

$$
\begin{aligned}
A_{1} & =\tilde{\Lambda}^{-1}\left\{a V^{-1} d x^{2}+\theta_{2} \omega^{34}+\theta_{3} \omega^{56}+\theta_{4} \omega^{78}\right\} \\
H_{3} & =-a \operatorname{dvol}\left(\mathbb{E}^{1,1}\right) \wedge d V^{-1} \\
H_{4} & =\operatorname{dvol}\left(\mathbb{E}^{1,1}\right) \wedge d x^{2} \wedge d V^{-1} \\
\Phi & =\frac{3}{4} \log \left(\tilde{\Lambda} \cdot V^{1 / 3}\right) .
\end{aligned}
$$

The configuration depends on an scalar function $\tilde{\Lambda}$ which is defined in terms of the scalar function $\Lambda$ appearing in the general discussion section, by

$$
\Lambda=V^{1 / 3} \cdot \tilde{\Lambda}
$$

and equals

$$
\begin{aligned}
\tilde{\Lambda}=1+a^{2} V^{-1}+\left(\theta_{2}\right)^{2} & \left(\left(x^{3}\right)^{2}+\left(x^{4}\right)^{2}\right) \\
& +\left(\theta_{3}\right)^{2}\left(\left(x^{5}\right)^{2}+\left(x^{6}\right)^{2}\right)+\left(\theta_{4}\right)^{2}\left(\left(x^{7}\right)^{2}+\left(x^{8}\right)^{2}\right) .
\end{aligned}
$$

To begin with, we shall give an interpretation for the configuration in which all transverse rotations are set to zero: $\theta_{i}=0$ for $i=2,3,4$. We 
would like to interpret it as the lift to ten dimensions of a bound state of $(p, q)$-strings in $N=2 D=9$ supergravity, or as the T-dual of $(p, q)$-strings in type IIB, this being the reason why fundamental strings are delocalised in the $x^{2}$ direction in the above supergravity solution. This interpretation can be inferred as follows. The global symmetries of supergravity theories compactified on torus have been studied extensively [77, 78]. Let us concentrate on $N=2 D=9$ obtained by reduction of $D=11$ on a 2 -torus. This ninedimensional theory is $\mathrm{SL}(2, \mathbb{R})$ invariant. What this means, among other things, is that configurations obtained from $D=11$ supergravity by reduction first along the $z$ direction, and afterwards, along the $x$ direction, can be mapped to those configurations in which one first reduces along the $x$ direction, and then on the $z$ direction. In this particular case of transverse directions, there is indeed an $\mathrm{SO}(2)$ transformation of angle $\pi / 2$ relating both configurations. There exist, of course, more general transformations. This is pretty close to what we have been doing. By reducing the M2-brane configuration along $\partial_{x}$ and $\partial_{z}$, or the other way around, we are describing $(p, 0)$ or $(0, q)$-strings in $N=2 D=9$. Under $\mathrm{SL}(2, \mathbb{R})$ transformations, one can generate the full spectrum of $(p, q)$-strings. Notice that these transformations are nothing but linear diffeomorphism transformations in $D=11$ supergravity which map $\partial_{z}$ into $a \partial_{z}+b \partial_{x}$, which is the kind of Killing vector we used to reduce the starting M2-brane configuration. Thus, the ten-dimensional configuration found above is nothing but the lift to ten dimensions of one of these $(p, q)$-strings, which is a bound state of D2-branes and delocalised fundamental strings.

Once this background has been understood, it is easy to interpret the effect of turning on the deformation parameters $\theta_{i}$. Indeed, whenever $\theta_{i} \neq 0$, the corresponding configurations are no longer asymptotically flat. They correspond to composite configurations of the vacuum and fluxbranes. The discussion of the different supersymmetric and non-supersymmetric possibilities is analogous to the ones already given before, so we refer the reader to the Table 6 summarising the results.

\begin{tabular}{|c|c|l|}
\hline$\nu$ & Object & Subalgebra \\
\hline \hline$\frac{1}{2}$ & D2-FA & $\{0\}$ \\
$\frac{1}{4}$ & $\mathrm{D} 2-\mathrm{FA}+\mathrm{F} 5$ & $\mathfrak{s u}(2)$ \\
$\frac{1}{8}$ & $\mathrm{D} 2-\mathrm{FA}+\mathrm{F} 3$ & $\mathfrak{s u}(3)$ \\
\hline
\end{tabular}

Table 6: Supersymmetric configurations of bound states made of D2-branes and delocalised fundamental strings (D2-FA) and fluxbranes. 
Let us move to the region of the moduli space where there is a nonvanishing null translation. In other words, we shall discuss the Kaluza-Klein reductions along the orbits of the Killing vectors $\xi=\partial_{z}+\alpha$ where

$$
\alpha=\partial_{+}+\theta_{2} R_{34}+\theta_{3} R_{56}+\theta_{4} R_{78} .
$$

The constant matrix $B$ is formally the same as in (3.28), but this time being written in the basis $\left\{x^{+}, x^{3}, \ldots, x^{7}, x^{8}\right\}$. Thus, besides the $z$ direction, it leaves invariant the $\left\{x^{-}, x^{2}, x^{9}\right\}$ directions. Since $\alpha$ involves a translation in the null direction $x^{+}$, there is a non-trivial 7-vector $\boldsymbol{C}$ describing the inhomogeneous part of the isometry transformation

$$
(\boldsymbol{C})^{t}=(1, \overrightarrow{0}) \text {. }
$$

The corresponding type IIA configurations have a ten-dimensional metric given by

$$
\begin{aligned}
g=\tilde{\Lambda}^{1 / 2}\left\{V^{-1 / 2}(\right. & \left.\left.2 d x^{+} d x^{-}+\left(d x^{2}\right)^{2}\right)+V^{1 / 2}\left(d s^{2}\left(\mathbb{E}^{6}\right)+\left(d x^{9}\right)^{2}\right)\right\} \\
& -\tilde{\Lambda}^{-1 / 2} V^{1 / 2}\left\{V^{-1} d x^{-}+\theta_{2} \omega^{34}+\theta_{3} \omega^{56}+\theta_{4} \omega^{78}\right\}^{2},
\end{aligned}
$$

whereas the RR 1-form $A_{1}$, NS-NS 3-form field strength $H_{3}, \mathrm{RR} 4$-form $H_{4}$ and dilaton $\Phi$ are listed below:

$$
\begin{aligned}
A_{1} & =\tilde{\Lambda}^{-1}\left\{V^{-1} d x^{-}+\theta_{2} \omega^{34}+\theta_{3} \omega^{56}+\theta_{4} \omega^{78}\right\} \\
H_{3} & =-d x^{-} \wedge d x^{2} \wedge d V^{-1} \\
H_{4} & =d x^{+} \wedge d x^{-} \wedge d x^{2} \wedge d V^{-1} \\
\Phi & =\frac{3}{4} \log \left(\tilde{\Lambda} \cdot V^{1 / 3}\right) .
\end{aligned}
$$

The configuration depends on an scalar function $\tilde{\Lambda}$ which is defined in terms of the scalar function $\Lambda$ appearing in the general discussion section, by

$$
\Lambda=V^{1 / 3} \cdot \tilde{\Lambda},
$$

and equals

$$
\tilde{\Lambda}=1+\left(\theta_{2}\right)^{2}\left[\left(x^{3}\right)^{2}+\left(x^{4}\right)^{2}\right]+\left(\theta_{3}\right)^{2}\left[\left(x^{5}\right)^{2}+\left(x^{6}\right)^{2}\right]+\left(\theta_{4}\right)^{2}\left[\left(x^{7}\right)^{2}+\left(x^{8}\right)^{2}\right] .
$$

Even though we lack a good physical understanding of the above set of configurations, it is instructive to look at the particular case in which $\theta_{i}=0$ for all $i$. We expect not to be describing any fluxbrane, and manage to isolate the new effect associated with the Kaluza-Klein reduction along an orbit involving lightlike translations. In such a case, the metric reduces to

$$
g=V^{-1 / 2} d s^{2}\left(\mathbb{E}^{1,2}\right)+V^{1 / 2} d s^{2}\left(\mathbb{E}^{7}\right)-V^{-3 / 2}\left(d x^{-}\right)^{2} .
$$


There is the standard RR 4-form giving the expected charge carried by a stack of D2-branes (and also the expected dilaton profile for a D2-brane configuration), but also non-trivial RR 1-form and NS-NS 3-form field strength given respectively by

$$
A_{1}=V^{-1} d x^{-} \quad \text { and } \quad H_{3}=-d x^{-} \wedge d x^{2} \wedge d V^{-1} .
$$

By inspection of the above expressions, it is clear that asymptotically at spacelike infinity $(r \rightarrow \infty)$, the solution is no longer Minkowski spacetime, but a wave background. Thus, just as fluxbranes induce some magnetic flux under Kaluza-Klein reduction and also modify the spacelike asymptotics, the extra lightlike translation induces the propagation of a lightlike perturbation at infinity. In the region $r \rightarrow 0$, one recovers the description close to a stack of D2-branes, in the first approximation. The stability and supersymmetry of the configuration requires both $H_{3}$ and $F_{2}=d A_{1}$ to be null forms.

By switching on the $\theta_{i}$ parameters, one expects to add fluxbranes to the above configuration. Since the discussion of the different possibilities does not give any new insight, we leave the details to the interested reader.

Let us finally consider the region of the moduli space which involves an extra timelike translation. That is, let us discuss the Kaluza-Klein reduction along the orbits of the Killing vector $\xi=\partial_{z}+\alpha$, where

$$
\alpha=a \partial_{0}+\theta_{2} R_{34}+\theta_{3} R_{56}+\theta_{4} R_{78} \quad, \quad|a|<1 .
$$

The constant matrix $B$ is again a $7 \times 7$ matrix given by (3.28) in the basis $\left\{x^{0}, x^{3}, \ldots, x^{7}, x^{8}\right\}$. Thus, it leaves the $\left\{x^{1}, x^{2}, x^{9}\right\}$ directions invariant. There is again a non-vanishing vector $\boldsymbol{C}$ given by

$$
(\boldsymbol{C})^{t}=(a, \overrightarrow{0}) \text {. }
$$

The corresponding type IIA configurations have a ten-dimensional metric given by

$$
\begin{aligned}
g=\tilde{\Lambda}^{1 / 2}\left\{V^{-1 / 2}\right. & \left.d s^{2}\left(\mathbb{E}^{1,2}\right)+V^{1 / 2} d s^{2}\left(\mathbb{E}^{7}\right)\right\} \\
& -\tilde{\Lambda}^{-1 / 2} V^{1 / 2}\left\{-a V^{-1} d x^{0}+\theta_{2} \omega^{34}+\theta_{3} \omega^{56}+\theta_{4} \omega^{78}\right\}^{2},
\end{aligned}
$$

whereas the RR 1-form $A_{1}$, NS-NS 3-form field strength $H_{3}$, RR 4-form $H_{4}$ and dilaton $\Phi$ are listed below:

$$
\begin{aligned}
A_{1} & =\tilde{\Lambda}^{-1}\left\{-V^{-1} a d x^{0}+\theta_{2} \omega^{34}+\theta_{3} \omega^{56}+\theta_{4} \omega^{78}\right\} \\
H_{3} & =-a d x^{1} \wedge d x^{2} \wedge d V^{-1} \\
H_{4} & =\operatorname{dvol}\left(\mathbb{E}^{1,2}\right) \wedge d V^{-1} \\
\Phi & =\frac{3}{4} \log \left(\tilde{\Lambda} \cdot V^{1 / 3}\right) .
\end{aligned}
$$


The configuration depends on an scalar function $\tilde{\Lambda}$ which is defined in terms of the scalar function $\Lambda$ appearing in the general discussion section, by

$$
\Lambda=V^{1 / 3} \cdot \tilde{\Lambda}
$$

and equals

$$
\begin{aligned}
\tilde{\Lambda}=1-V^{-1} a^{2}+\left(\theta_{2}\right)^{2} & {\left[\left(x^{3}\right)^{2}+\left(x^{4}\right)^{2}\right] } \\
& +\left(\theta_{3}\right)^{2}\left[\left(x^{5}\right)^{2}+\left(x^{6}\right)^{2}\right]+\left(\theta_{4}\right)^{2}\left[\left(x^{7}\right)^{2}+\left(x^{8}\right)^{2}\right] .
\end{aligned}
$$

Let us start by considering the particular configuration in which all transverse rotation parameters vanish: $\theta_{i}=0$ for $i=2,3,4$. The role played by the bound $|a|<1$ can be immediately appreciated by inspection of the corresponding metric

$$
g=-\left(V-a^{2}\right)^{-1 / 2}\left(d x^{0}\right)^{2}+\left(V-a^{2}\right)^{1 / 2}\left\{d s^{2}\left(\mathbb{E}^{7}\right)+V^{-1} d s^{2}\left(\mathbb{E}^{2}\right)\right\} .
$$

It is now clear that the condition $|a|<1$ ensures the absence of horizons in spacetime. Furthermore, if $|a| \geq 1$ would have been arbitrary, these horizons sitting at

$$
r_{\mathrm{H}}^{5}=\frac{Q}{a^{2}-1}
$$

would have divided spacetime into regions $\left(r>r_{\mathrm{H}}\right)$ having closed timelike curves and regions $\left(r<r_{\mathrm{H}}\right)$ free of this causal sickness.

Despite these features, the physical interpretation of these configurations remains unclear. Proceeding as in previous configurations involving more than one spacelike translation, it would be natural to interpret this configuration as a bound state of D2-branes and delocalised E2-branes [79, 80, 81], the charge of the latter being constrained by the bound $|a|<1$. It is clear that the geometry in the region $r \rightarrow 0$ is the one describing the core of a stack of D2-branes, whereas in the asymptotic spacelike infinity, this time we recover Minkowski spacetime by a trivial rescaling of coordinates (notice that the dilaton acquires a constant factor depending on $a$ in this asymptotic limit).

Even though this configuration has no clear physical interpretation, it is obvious that it allows the addition of fluxbranes by switching on the $\theta_{i}$ parameters, while preserving some supersymmetry. Since the discussion of these possibilities does not involve any new features, we leave the details to the reader. 


\section{Kaluza-Klein reductions of the M5-brane}

In this section we classify the set of M-theory backgrounds obtained by modding out the M5-brane background by a one-parameter subgroup of its isometry group and study the corresponding smooth supersymmetric Kaluza-Klein reductions along the orbits of the Killing vectors generating such subgroups. We shall first describe the standard M5-brane configuration in section 4.1. Afterwards, we shall discuss the M5-brane delocalised along one transverse direction in section 4.2 .

\subsection{Supersymmetric reductions of the M5-brane}

The M-theory fivebrane [82] is described by a metric of the type (1.7) with two factors,

$$
g=V^{-1 / 3} d s^{2}\left(\mathbb{E}^{1,5}\right)+V^{2 / 3} d s^{2}\left(\mathbb{E}^{5}\right),
$$

where $V=1+|Q| / r^{3}$ with $|Q|$ some positive constant and $r$ the radial distance in the transverse $\mathbb{E}^{5}$. The 7 -form dual to the 4 -form is given by

$$
* F_{4}=\operatorname{dvol}\left(\mathbb{E}^{1,5}\right) \wedge d V^{-1}
$$

up to a constant of proportionality. The Killing spinors are of the form

$$
\varepsilon=V^{-1 / 12} \varepsilon_{\infty},
$$

where $\varepsilon_{\infty}$ is a constant spinor satisfying

$$
\operatorname{dvol}\left(\mathbb{E}^{1,5}\right) \cdot \varepsilon_{\infty}=\varepsilon_{\infty} .
$$

The symmetry group is

$$
G=\mathrm{ISO}(1,5) \times \mathrm{SO}(5) \subset \mathrm{ISO}(1,10),
$$

with Lie algebra

$$
\mathfrak{g}=\left(\mathbb{R}^{1,5} \rtimes \mathfrak{s o}(1,5)\right) \times \mathfrak{s o}(5),
$$

whence any Killing vector $\xi$ can be decomposed as

$$
\xi=\tau_{\|}+\lambda_{\|}+\rho_{\perp}
$$

with the usual notation. 


\subsubsection{Freely-acting spacelike isometries}

As before, to determine the freely-acting spacelike Killing vectors, we exploit the freedom to conjugate by $G$ in order to bring $\xi$ to a convenient normal form. Conjugating first by the $\mathrm{SO}(1,5)$ subgroup, we can bring $\lambda$ to one of several normal forms. Either $\lambda=0$ or else it is conjugate to one of the following three normal forms

1. $\lambda=\beta B_{01}+\theta_{1} R_{23}+\theta_{2} R_{45}$, with $\beta \neq 0$;

2. $\lambda=N_{+2}+\theta R_{45}$; or

3. $\lambda=\theta_{1} R_{23}+\theta_{2} R_{45}$,

with the same notation introduced earlier. The first case can be easily discarded since for $\beta \neq 0, \xi$ is not everywhere spacelike, regardless what $\rho_{\perp}$ and $\tau$ are. Changing the origin in the worldvolume of the brane, it is possible to set $\tau$ in the second case to $a \partial_{-}+b \partial_{3}$; but again unless $a=0, \xi$ will not be everywhere spacelike. For a freely-acting $\xi$ one must in addition have $\tau \neq 0$. Similarly in the third and final case, $\tau$ must be spacelike for $\xi$ to be everywhere spacelike, hence we can conjugate $\tau$ to a spacelike direction orthogonal to $\lambda$, say $\tau \propto \partial_{1}$, where again for a free action $\tau \neq 0$. In this case the vector fields all integrate to a free action because of the presence of the translation. In summary, we have three possible cases of freely-acting spacelike Killing vectors in the M5-brane geometry:

(A) $\xi=\tau_{\|}+\rho_{\|}+\rho_{\perp}$, with $\tau \neq 0$ spacelike and where $\rho_{\|}$can vanish; and

(B) $\xi=\tau_{\|}+\nu_{\|}+\rho_{\|}+\rho_{\perp}$, with $\nu_{\|} \neq 0, \tau \neq 0$ spacelike, and where $\rho_{\|}$can vanish.

We again remark that the above decompositions of $\xi$ are orthogonal relative to the brane metric.

\subsubsection{Moduli space of smooth reductions}

In case (A) above, the Killing vector $\xi$ can be brought to the form

$$
\xi=a \partial_{1}+\theta_{1} R_{23}+\theta_{2} R_{45}+\theta_{3} R_{67}+\theta_{4} R_{89}
$$

with $a \neq 0$. There are five free parameters, which after projectivisation and modding out by the action of the Weyl group yields a four-dimensional 
moduli space of smooth reductions and within it a three-dimensional supersymmetric locus.

In case (B), the Killing vector $\xi$ can be brought to the form

$$
\xi=a \partial_{1}+N_{+3}+\theta_{2} R_{45}+\theta_{3} R_{67}+\theta_{4} R_{89},
$$

where $a \neq 0$. There is now a three-dimensional moduli space of smooth reductions and supersymmetry will select a two-dimensional locus.

\subsubsection{Supersymmetry}

In both of the above cases the rotation component of the Killing vector $\xi$ takes the general form

$$
\rho=\theta_{1} R_{23}+\theta_{2} R_{45}+\theta_{3} R_{67}+\theta_{4} R_{89} .
$$

Relative to a basis dual to the $R_{i j}$ the weights of the subspace $S_{0}$ of the half-spin representation of $\operatorname{Spin}(1,10)$ obeying $(4.4)$ are given in equation (A.3). The supersymmetric locus is therefore the union of eight hyperplanes

$$
\sum_{i=1}^{4} \mu_{i} \theta_{i}=0, \quad \text { where } \mu_{i}^{2}=1 .
$$

(Again there are only eight hyperplanes, because the weights $\mu$ and $-\mu$ determine the same hyperplane.) A rotation $\rho$ belonging to one and only one of these hyperplanes belongs to an $\mathfrak{s u}(4)$ subalgebra. Two weights will annihilate such a rotation and hence the associated reduction will preserve a fraction $\nu=\frac{1}{16}$ of the supersymmetry. Points which lie in the intersection of two hyperplanes come in two flavours: those points where no $\theta$ vanish, which belong to an $\mathfrak{s p}(1) \times \mathfrak{s p}(1)$ subalgebra and those for which one of the $\theta$ s vanish, which belong to an $\mathfrak{s u}(3)$ subalgebra. In either case, such a rotation is annihilated by four weights and hence the reduction will preserve a fraction $\nu=\frac{1}{8}$ of the supersymmetry. Points which lie in the intersection of three hyperplanes necessarily have two vanishing $\theta \mathrm{s}$ and they belong to an $\mathfrak{s u}(2)$ subalgebra and their reductions preserve a fraction $\nu=\frac{1}{4}$ of the supersymmetry. Finally the only point which lies in the intersection of four hyperplanes (and hence in all hyperplanes) is the origin. This reduction preserves all the supersymmetry of the M5-brane, hence a fraction $\nu=\frac{1}{2}$. This concludes the analysis of case (A). Case (B) corresponds to setting $\theta_{1}=0$ and introducing a null rotation, whence the supersymmetry is further halved. There are now four hyperplanes in the subspace $\theta_{1}=0$. The generic points belong to an $\mathfrak{s u}(3)$ subalgebra and their reductions preserve a fraction 
$\nu=\frac{1}{16}$ of the supersymmetry. Points in the intersection of two hyperplanes belong to an $\mathfrak{s u}(2)$ subalgebra and preserve a fraction $\nu=\frac{1}{8}$. Finally, the only point in the intersection of three hyperplanes is the origin, which preserves a fraction $\nu=\frac{1}{4}$ of the supersymmetry. This is summarised in Table 7 .

Notice that by constructing $\mathcal{M}_{\mathrm{M} 5} / \Gamma_{0}$, where $\Gamma_{0} \subset \Gamma$ is a discrete subgroup, the previous classification gives rise to a whole set of smooth supersymmetric eleven-dimensional configurations. This set includes a stack of M5-branes and eleven-dimensional fluxbranes $\left(\xi=\partial_{1}+\rho\right)$ or elevendimensional nullbranes $\left(\xi=\partial_{1}+\nu_{\|}\right)$.

\begin{tabular}{|c|c|c|c|}
\hline Null rotation? & Subalgebra & $\nu$ & $\operatorname{dim}$ \\
\hline \hline \multirow{5}{*}{ No } & $\mathfrak{s u}(4)$ & $\frac{1}{16}$ & 3 \\
& $\mathfrak{s u}(3)$ & $\frac{1}{8}$ & 2 \\
& $\mathfrak{s p}(1) \times \mathfrak{s p}(1)$ & $\frac{1}{8}$ & 2 \\
& $\mathfrak{s u}(2)$ & $\frac{1}{4}$ & 1 \\
& $\{0\}$ & $\frac{1}{2}$ & 0 \\
\hline \multirow{5}{*}{ Yes } & $\mathfrak{s u}(3)$ & $\frac{1}{16}$ & 2 \\
& $\mathfrak{s u}(2)$ & $\frac{1}{8}$ & 1 \\
& $\{0\}$ & $\frac{1}{4}$ & 0 \\
\hline
\end{tabular}

Table 7: Supersymmetric reductions of the M5-brane. All translations are spacelike and tangent to the M5-brane. We indicate the spinor isotropy subalgebra to which the rotation belongs, the fraction $\nu$ of the supersymmetry preserved and the dimension of the corresponding stratum of the moduli space $\mathcal{M}$ of supersymmetric reductions.

\subsubsection{Explicit reductions}

It is possible to discuss the full set of inequivalent Kaluza-Klein reductions of the M5-brane by a single computation, the one associated with reductions along the orbits of the Killing vector $\xi=\partial_{z}+\lambda$, where

$$
\lambda=\beta B_{03}+\theta_{1} R_{23}+\theta_{2} R_{45}+\theta_{3} R_{67}+\theta_{4} R_{89},
$$

and $z$ stands for the $x^{1}$ direction along the M5-brane.

The constant matrix $B$ is a $9 \times 9$ matrix, which does not act on the $x^{\natural}$ coordinate and which equals, formally, the one appearing in (3.25), but this 
time in the basis $\left\{x^{0}, x^{2}, \ldots, x^{9}\right\}$. The ten-dimensional metric obtained after Kaluza-Klein reduction can be written as,

$$
\begin{aligned}
g=\tilde{\Lambda}^{1 / 2}\left\{V^{-1 / 2} d s^{2}\left(\mathbb{E}^{1,4}\right)+V^{1 / 2} d s^{2}\left(\mathbb{E}^{5}\right)\right\} \\
-\tilde{\Lambda}^{-1 / 2} V^{-1 / 2}\left\{\beta \omega^{03}+\theta_{1} \omega^{23}+\theta_{2} \omega^{45}+V\left[\theta_{3} \omega^{67}+\theta_{4} \omega^{89}\right]\right\}^{2},
\end{aligned}
$$

whereas the RR 1-form $A_{1}, \mathrm{RR} 4$-form $H_{4}$ and dilaton $\Phi$ are listed below:

$$
\begin{aligned}
A_{1} & =\tilde{\Lambda}^{-1}\left\{\beta \omega^{03}+\theta_{1} \omega^{23}+\theta_{2} \omega^{45}+V\left[\theta_{3} \omega^{67}+\theta_{4} \omega^{89}\right]\right\} \\
H_{4} & =-\star \operatorname{dvol}\left(\mathbb{E}^{1,4}\right) \wedge d V^{-1} \\
\Phi & =\frac{3}{4} \log \left(\tilde{\Lambda} \cdot V^{-1 / 3}\right) .
\end{aligned}
$$

The configuration depends on an scalar function $\tilde{\Lambda}$ which is defined in terms of the scalar function $\Lambda$ appearing in the general discussion section, by

$$
\Lambda=V^{-1 / 3} \cdot \tilde{\Lambda}
$$

and equals

$$
\begin{aligned}
\tilde{\Lambda}=1+\left(x^{3}\right)^{2}\left[\left(\theta_{1}\right)^{2}-\beta^{2}\right]+\left(\beta x^{0}+\theta_{1} x^{2}\right)^{2}+\left(\theta_{2}\right)^{2}\left[\left(x^{4}\right)^{2}+\left(x^{5}\right)^{2}\right] \\
+V\left\{\left(\theta_{3}\right)^{2}\left[\left(x^{6}\right)^{2}+\left(x^{7}\right)^{2}\right]+\left(\theta_{4}\right)^{2}\left[\left(x^{8}\right)^{2}+\left(x^{9}\right)^{2}\right]\right\} .
\end{aligned}
$$

As discussed for the M2-brane reductions, whenever $\left|\theta_{1}\right|<|\beta|$, there is always a Lorentz observer who sees a pure boost. Such spacetime breaks supersymmetry and contains closed timelike curves. If we restrict to the regions of spacetime where such closed causal curves do not exist, their interpretation would give rise to similar cosmological models to the ones discussed in $[45,46,48,49]$ but this time on the worldvolume of a $1+5$ brane. On the other hand, whenever $\left|\theta_{1}\right|>|\beta|$, there is always an observer who measures a pure rotation, so that case would be related to fluxbranes.

Let us concentrate on the interpretation of the different regions of the above reduction. If we set $\beta=0$, there are five different possibilities to be considered:

(1) The case $\theta_{i}=0$ for all $i$ corresponds to the well-known D4-brane in type IIA preserving $\nu=1 / 2$ of the spacetime supersymmetry.

(2) If one of the $\theta \mathrm{s}$ is non-vanishing, the configuration describes a composite state involving a D4-brane and an F7-brane. Depending on the chosen $\theta$, its location is a (456789 )-plane at $x^{3}=x^{4}=0$ (if $\theta_{1} \neq 0$ ) or a (234589 )-plane at $x^{6}=x^{7}=0$ (if $\theta_{2} \neq 0$ ). In either case, supersymmetry is completely broken due to the presence of these F7-branes. 
(3) If there are two non-vanishing $\theta$ s we must distinguish between four cases:

(i) If $\theta_{1}=\eta_{2} \theta_{2}$, the configuration describes a $\mathrm{D} 4$-brane in the (2345)plane and an F5-brane along the (6789h)-plane sitting at $x^{2}=$ $x^{3}=x^{4}=x^{5}=0$. It preserves $\nu=1 / 4$ of the spacetime supersymmetry, with Killing spinors being preserved by an $\mathfrak{s u}(2)$ subalgebra.

(ii) If $\theta_{3}=\eta_{4} \theta_{4}$, the configuration describes a D4-brane in the (2345)plane and an F5-brane along the (2345 ) )-plane sitting at $x^{6}=$ $x^{7}=x^{8}=x^{9}=0$. It preserves $\nu=1 / 4$ of the spacetime supersymmetry, with Killing spinors being preserved by an $\mathfrak{s u}(2)$ subalgebra.

(iii) If $\theta_{1}=\eta_{3} \theta_{3}$, the configuration describes a D4-brane in the (2345)plane and an F5-brane along the (4589h)-plane sitting at $x^{2}=$ $x^{3}=x^{6}=x^{7}=0$. It preserves $\nu=1 / 4$ of the spacetime supersymmetry, with Killing spinors again preserved by an $\mathfrak{s u}(2)$ subalgebra.

(iv) If $\theta_{i} \neq \eta_{j} \theta_{j} \mathrm{i} \neq \mathrm{j}$, the configuration describes a system of two intersecting F7-branes besides the aforementioned D4-brane. It breaks supersymmetry completely.

(4) When there are three non-vanishing $\theta$ s, we must distinguish between three cases:

(i) If $\theta_{1}=\eta_{2} \theta_{2}+\eta_{3} \theta_{3}$, the configuration describes a $\mathrm{D} 4$-brane in the (2345)-plane and an F3-brane along the (89h)-plane sitting at $x^{k}=0 k=2, \ldots, 7$. It preserves $\nu=1 / 8$ of the spacetime supersymmetry, with Killing spinors being preserved by an $\mathfrak{s u}(3)$ subalgebra.

(ii) If $\theta_{2}=\eta_{3} \theta_{3}+\eta_{4} \theta_{4}$, the F3-brane extends along the (23ฤ)-plane sitting at $x^{k}=0 k=4, \ldots, 9$. It preserves the same amount of supersymmetry as the previous one due to the existence of an $\mathfrak{s u ( 3 )}$ subalgebra preserving some Killing spinors.

(iii) If $\theta_{i} \neq \eta_{j} \theta_{\jmath}+\eta_{k} \theta_{k}$ for all $i, j, k$ distinct, the configuration describes the intersection of three different F7-branes plus a D4brane. Spacetime supersymmetry is completely broken.

(5) When four $\theta$ s are nonvanishing, there are generically two inequivalent ways of preserving Killing spinors, but due to the symmetries of our configuration, these split into four: 
(i) If $\theta_{1}=\eta_{2} \theta_{2}+\eta_{3} \theta_{3}+\eta_{4} \theta_{4}$, the configuration describes a D4brane in the (2345)-plane and a flux string along the $x^{\natural}$ direction, preserving $\nu=1 / 16$ of the spacetime supersymmetry. This is the one associated with the $\mathfrak{s u}(4)$ isotropy algebra discussed before.

(ii) If $\theta_{1}=\eta_{2} \theta_{2}$ and $\theta_{3}=\eta_{4} \theta_{4}$, the configuration involves a D4-brane and a $\frac{1}{4}$-BPS fluxstring in the $x^{\natural}$ direction. It preserves $\nu=1 / 8$ and it is associated with the $\mathfrak{s p}(1) \times \mathfrak{s p}(1)$ isotropy algebra.

(iii) The case $\theta_{1}=\eta_{3} \theta_{3}$ and $\theta_{2}=\eta_{4} \theta_{4}$, has the same interpretation as the previous one, but the $\mathfrak{s p}(1) \times \mathfrak{s p}(1)$ isotropy algebra is selected in a different way.

(iv) If non of the three previous possibilities are satisfied, there are four intersecting F7-branes and a stack of coincident D4-branes breaking spacetime supersymmetry completely.

If $\beta \neq 0$, the only allowed possibility preserving supersymmetry requires $\left|\theta_{1}\right|=|\beta|$. Let us thus concentrate on this case. Depending on whether the remaining parameters vanish or satisfy certain linear relations, we distinguish between the following configurations

(1) If $\theta_{i}=0$ for $i=2,3,4$, it describes a D4-brane in the (2345)-plane and a nullbrane. This composite configuration preserves $\nu=1 / 4$ of the spacetime supersymmetry.

(2) If one of the $\theta$ s is non-vanishing, the corresponding configuration breaks supersymmetry completely, due to the presence of an F7-brane besides the previous $\mathrm{D} 4 /$ nullbrane pair.

(3) If two of the $\theta$ s are non-vanishing, we shall distinguish between three cases:

(i) If $\theta_{i} \neq \eta_{j} \theta_{j} i \neq j$, the configuration describes a system of two intersecting F7-branes (besides the composite system of a D4brane and a nullbrane), such that all supersymmetry is broken.

(ii) If $\theta_{2}=\eta_{3} \theta_{3}$, the intersection among the $\mathrm{F} 7$-branes gives rise to the so-called F5-brane, extending along the (2389 )-plane and sitting at $x^{4}=x^{5}=x^{6}=x^{7}=0$. The full configuration includes the previous pair D4/nullbrane system, thus preserving $\nu=1 / 8$.

(iii) If $\theta_{3}=\eta_{4} \theta_{4}$, one finds a second F5-brane, this time extending along the 2345 -plane and sitting at $x^{6}=x^{7}=x^{8}=x^{9}=0$. As before, the configuration preserves $\nu=1 / 8$.

(4) If all $\theta$ s are non-vanishing, we need to distinguish between two cases: 
(i) If $\theta_{2} \neq \eta_{3} \theta_{3}+\eta_{4} \theta_{4}$, the configuration describes the intersection of three intersecting F7-branes, besides the composite D4/nullbrane system, thus breaking all supersymmetry.

(ii) If $\theta_{2}=\eta_{3} \theta_{3}+\eta_{4} \theta_{4}$, the intersection of the F7-branes gives rise to an F3-brane extending along the (23দ)-plane and sitting at $x^{k}=0$ $\mathrm{k}=4, \ldots, 9$. Thus, there exists a composite configuration involving a D4-brane, a nullbrane and an F3-brane preserving $\nu=1 / 16$.

The set of supersymmetric configurations described above is summarised in Table 8, where the notation $\mathrm{Dp} \perp \mathrm{Fq}(r)$ has been introduced. The latter stands for a $\mathrm{D} p$-brane $+\mathrm{F} q$-brane composite configuration sharing $r$ spacelike directions.

\begin{tabular}{|c|c|l|}
\hline$\nu$ & Object & Subalgebra \\
\hline \hline & $\mathrm{D} 4 \perp \mathrm{F} 5(0)$ & \\
$\frac{1}{4}$ & $\mathrm{D} 4 \perp \mathrm{F} 5(4)$ & $\mathfrak{s u}(2)$ \\
& $\mathrm{D} 4 \perp \mathrm{F} 5(2)$ & \\
$\frac{1}{4}$ & $\mathrm{D} 4+\mathrm{N}$ & $\mathbb{R}$ \\
$\frac{1}{8}$ & $\mathrm{D} 4 \perp \mathrm{F} 3(0)$ & $\mathfrak{s u}(3)$ \\
& $\mathrm{D} 4 \perp \mathrm{F} 3(2)$ & \\
$\frac{1}{8}$ & $(\mathrm{D} 4 \perp \mathrm{F} 5(4))+\mathrm{N}$ & $\mathfrak{s u}(2) \times \mathbb{R}$ \\
& $(\mathrm{D} 4 \perp \mathrm{F} 5(2))+\mathrm{N}$ & \\
$\frac{1}{8}$ & $\mathrm{D} 4 \perp \mathrm{F} 1(0)$ & $\mathfrak{s p}(1) \times \mathfrak{s p}(1)$ \\
$\frac{1}{16}$ & $\mathrm{D} 4 \perp \mathrm{F} 1(0)$ & $\mathfrak{s u}(4)$ \\
$\frac{1}{16}$ & $(\mathrm{D} 4 \perp \mathrm{F} 3(2))+\mathrm{N}$ & $\mathfrak{s u}(3) \times \mathbb{R}$ \\
\hline
\end{tabular}

Table 8: Supersymmetric configurations of D4-branes, fluxbranes and nullbranes.

Before finishing this presentation, we would like to compute the fluxes associated with F5-branes in the presence of D4-branes. There are three cases to be considered separately, as indicated in Table 8, and discussed in the text above. Whenever the D4-branes are parallel to the F5-brane, $\theta_{1}=\theta_{2}=\beta=0, \theta \equiv \theta_{3}=\theta_{4}$, the RR 1-form potential in (4.11) depends on the direction $x^{\natural}$ along the F5-brane but transverse to the D4-branes. As it happened for fundamental strings, it can be shown that the flux carried by the F5-brane equals the one on flat spacetime, except at $x^{\natural}=0$, where it vanishes. It is precisely at $x^{\natural}=0$, where the D4-branes lie. A probe 
computation shows that indeed D4-branes with the above orientation are only stable where the flux vanishes. On the other extreme, when the D4branes are completely tranverse to the F5-branes $\theta_{3}=\theta_{4}=\beta=0, \theta \equiv \theta_{1}=$ $\theta_{2}$, the RR 1-form potential in (4.11) is independent of the worldvolume F5-brane point. Therefore the flux equals the one carried in flat spacetime everywhere. Finally, when they are relatively transverse, $\theta_{2}=\theta_{4}=\beta=$ $0, \theta \equiv \theta_{1}=\theta_{3}$, the RR 1-form potential in (4.11) depends on the radial distance in the 89 -plane spanned by the F5-brane. By fixing a point in this plane, it can be shown that

$$
F_{2} \wedge F_{2} \propto-2 \frac{\partial\left(r_{1} \tilde{\Lambda}^{-2}\right)}{\partial r_{2}} d r_{1} \wedge d \theta_{1} \wedge d r_{2} \wedge d \theta_{2},
$$

where we used the same parametrisation as for the corresponding M2-brane discussion. Its integral over $\mathbb{R}^{4}$ equals the one in flat spacetime everywhere except at the origin of the (89ø)-plane, where it vanishes.

\subsection{Supersymmetric reductions of the delocalised M5-brane}

To obtain the NS5-brane by Kaluza-Klein reduction of the M5-brane, it is necessary to delocalise the M5-brane along a transverse direction. In this section we will classify the supersymmetric Kaluza-Klein reductions of such a delocalised M5-brane. The metric of the spacetime exterior to such a fivebrane is again of the general form (1.7) but now with three factors:

$$
g=V^{-1 / 3} d s^{2}\left(\mathbb{E}^{1,5}\right)+V^{2 / 3} d z^{2}+V^{2 / 3} d s^{2}\left(\mathbb{E}^{4}\right),
$$

where $z$ is the transverse coordinate along which the fivebrane is delocalised and $V=1+|Q| / r^{2}$ is a harmonic function on $\mathbb{E}^{4}$ depending only on the radial distance. The symmetry group is now

$$
G=\operatorname{ISO}(1,5) \times \mathbb{R} \times \operatorname{SO}(4),
$$

with Lie algebra

$$
\mathfrak{g}=\left(\mathbb{R}^{1,5} \rtimes \mathfrak{s o}(1,5)\right) \times \mathbb{R} \times \mathfrak{s o}(4) .
$$

Therefore a Killing vector may be decomposed as

$$
\xi=\tau_{\|}+\tau_{\perp}+\lambda_{\|}+\rho_{\perp}
$$

in the usual notation. 


\subsubsection{Freely-acting spacelike isometries}

As for the localised fivebrane, either the Lorentz transformation $\lambda=0$ or else it can be brought to one the following normal forms:

1. $\lambda=\beta B_{01}+\theta_{1} R_{23}+\theta_{2} R_{45}$, with $\beta \neq 0$;

2. $\lambda=N_{+2}+\theta R_{45} ;$ or

3. $\lambda=\theta_{1} R_{23}+\theta_{2} R_{45}$.

It is again easy to discard the first case, since $\beta \neq 0$ means that $\xi$ is not everywhere spacelike. In the second case, $\tau_{\|}$is spacelike and $\tau_{\|}+\tau_{\perp}$ cannot be zero, because otherwise the action is not free: as in the M2 case, there would be points outside the horizon with nontrivial stabilisers. As in the M2-brane, the norm of the transverse rotation $\rho_{\perp}$ obeys a sharp bound

$$
r^{2} M^{2} \geq\left\|\rho_{\perp}\right\|_{\infty}^{2} \geq r^{2} m^{2},
$$

where $M \geq m \geq 0$ and $m$ can be nonzero since the transverse sphere is three-dimensional and possesses infinitesimal isometries without zeros. This means that in the third case, the norm of the Killing vector is bounded below by

$$
\|\xi\|^{2} \geq V^{-1 / 3}\left(\left\|\tau_{\|}\right\|_{\infty}^{2}+\left\|\rho_{\|}\right\|_{\infty}^{2}\right)+V^{2 / 3}\left(\left\|\tau_{\perp}\right\|_{\infty}^{2}+r^{2} m^{2}\right)
$$

which is again sharp. Because there are points where $\rho_{\|}=0$, the bound can be improved to

$$
\|\xi\|^{2} \geq V^{-1 / 3}\left\|\tau_{\|}\right\|_{\infty}^{2}+V^{2 / 3}\left(\left\|\tau_{\perp}\right\|_{\infty}^{2}+r^{2} m^{2}\right),
$$

which is still sharp. The right-hand side of the above bound defines a function $f$ of $r$ with the following asymptotic properties. As $r \rightarrow 0$,

$$
f(r)=|Q|^{2 / 3}\left\|\tau_{\perp}\right\|_{\infty}^{2} r^{-4 / 3}+O\left(r^{2 / 3}\right),
$$

whence it blows up if $\tau_{\perp} \neq 0$ and goes to zero otherwise. In the asymptotic regime where $r \rightarrow \infty$,

$$
f(r)=m^{2} r^{2}+\left\|\tau_{\|}\right\|_{\infty}^{2}+\left\|\tau_{\perp}\right\|_{\infty}^{2}+O\left(r^{-1}\right),
$$

which blows up for $m>0$, and approaches (the square of) the flat norm of $\tau_{\|}+\tau_{\perp}$ otherwise. Therefore, generically $f$ has a minimum at some critical value $r_{0}>0$; although if either $\tau_{\perp}$ or $m$ vanish this may be either zero or not exist, respectively. As in the case of the M2-brane, there is a positive 
number $\mu$ such that $\xi$ is everywhere spacelike if and only if $\left\|\tau_{\|}\right\|_{\infty}^{2}>-\mu^{2}$. This number is obtained by solving $f^{\prime}\left(r_{0}\right)=0$, which gives $\mu$ as a function of $r_{0}$, and substituting this into $f\left(r_{0}\right)=0$ which can be solved for $r_{0}$ and hence for $\mu$. One finds that the critical radius obeys

$$
r_{0}^{4} m^{2}=|Q|\left\|\tau_{\perp}\right\|_{\infty}^{2},
$$

should one exist (it does if $m>0$ ) and hence that

$$
\mu=\left\|\tau_{\perp}\right\|_{\infty}+m|Q|^{1 / 2} .
$$

If $m=0$ we see that it is enough that $\tau_{\|}+\tau_{\perp}$ be asymptotically spacelike.

In summary, the freely-acting spacelike Killing vectors of the delocalised fivebrane geometry fall into two cases:

(A) $\xi=\tau_{\|}+\tau_{\perp}+\nu_{\|}+\rho_{\|}+\rho_{\perp}$, with $\nu_{\|} \neq 0, \tau_{\|}+\tau_{\perp}$ spacelike and where $\tau_{\|}$if nonzero must also be spacelike; and

(B) $\xi=\tau_{\|}+\tau_{\perp}+\rho_{\|}+\rho_{\perp}$, with $\tau_{\|}$satisfying a norm constraint of the form $\left\|\tau_{\|}\right\|_{\infty}^{2}>-\mu^{2}$, where $\mu$ is given by equation (4.17). In particular, if $\rho_{\perp}$ has zeros, then $\tau_{\|}+\tau_{\perp}$ must be asymptotically spacelike.

The rotation $\rho_{\|}$is allowed to vanish in both cases and, once again, the decompositions of $\xi$ are orthogonal with respect to the brane metric. In both cases, the translation $\tau_{\|}+\tau_{\perp}$ must be nonzero for the action of $\xi$ to be free.

\subsubsection{Moduli space of smooth reductions}

In case (A) we can always choose coordinates so that the Killing vector $\xi$ takes the form

$$
\xi=a \partial_{1}+b \partial_{z}+N_{+2}+\theta_{2} R_{45}+\theta_{3} R_{67}+\theta_{4} R_{89},
$$

where $a$ and $b$ cannot both be zero. There are five free parameters which yield a four-dimensional moduli space of smooth reductions after we projectivise and quotient by the action of the (discrete) Weyl group. Supersymmetry will then select a three-dimensional locus.

Case (B) breaks up into three cases depending on the nature of $\tau_{\|}$: whether it is timelike, spacelike or null. In the first case we can bring $\xi$ to the form

$$
\xi=a \partial_{0}+b \partial_{z}+\theta_{1} R_{23}+\theta_{2} R_{45}+\theta_{3} R_{67}+\theta_{4} R_{89}
$$


which gives rise to a five-dimensional moduli space of smooth reductions with a four-dimensional supersymmetric locus. The results are similar for $\tau_{\|}$spacelike. Finally, if $\xi$ is null then we can always bring it to the form

$$
\xi=\partial_{+}+b \partial_{z}+\theta_{1} R_{23}+\theta_{2} R_{45}+\theta_{3} R_{67}+\theta_{4} R_{89}
$$

whence we have one less parameter. Therefore the moduli space of smooth reductions will be four-dimensional with a codimension-one locus of supersymmetric reductions.

\subsubsection{Absence of closed causal curves}

It is rather straightforward to extend the proofs of absence of closed causal curves given for the quotients of the M2-brane background to the M5-brane background discussed here. As in that case, we shall set all $\theta_{i}$ and $\beta$ to zero.

Let us start by analysing the problem of existence of closed causal curves in an M5-brane background delocalised in one transverse direction $(z)$ reduced along the orbits of the Killing vector

$$
\xi=a \partial_{0}+b \partial_{z} .
$$

The only condition that such a Killing vector is required to satisfy is to be spacelike everywhere. This requirement provides us with the constraint

$$
\|\xi\|^{2}=V(r)^{-1 / 3}\left(-a^{2}+V(r) b^{2}\right)>0 .
$$

Writing the metric in an adapted coordinate system, in which $\xi=\partial_{z^{\prime}}$, one finds

$$
\begin{aligned}
g=-V^{-1 / 3}\left[a^{2}\left(d z^{\prime}\right)^{2}+b^{-2}\left(d t^{\prime}\right)^{2}+2 a b^{-1} d z^{\prime} d t^{\prime}\right]+b^{2} V^{2 / 3}\left(d z^{\prime}\right)^{2} \\
+V^{-1 / 3} d s^{2}\left(\mathbb{E}^{5}\right)+V^{2 / 3} d s^{2}\left(\mathbb{E}^{4}\right) .
\end{aligned}
$$

Let us assume the existence of causal curves $x(\lambda)$, i.e. $\left\|\frac{d x}{d \lambda}\right\|^{2} \leq 0$, joining the points $\left(t_{0}^{\prime}, x_{0}^{i}, z_{0}^{\prime}\right)$ and $\left(t_{0}^{\prime}, x_{0}^{i}, z_{0}^{\prime}+\Delta\right)$. As argued for the M2-brane background, there must exist at least one value of the affine parameter $\lambda$ where the timelike component of the tangent vector to the curve vanishes:

$$
\exists \lambda^{*} \text { such that }\left.\frac{d t^{\prime}}{d \lambda}\right|_{\lambda^{*}}=0 .
$$


If one computes the norm of such a tangent vector at $\lambda^{*}$, one derives the inequality

$$
\left.\|\xi\|^{2}\left(\lambda^{*}\right) \frac{d z^{\prime}}{d \lambda}\right|_{\lambda^{*}} ^{2}+\left.V^{-1 / 3} \sum_{i=1}^{5} \frac{d x^{i}}{d \lambda}\right|_{\lambda^{*}} ^{2}+\left.V^{2 / 3} \sum_{i=6}^{9} \frac{d x^{i}}{d \lambda}\right|_{\lambda^{*}} ^{2} \leq 0 .
$$

Due to the constraint (4.18), it is clear that the left hand side of the above norm is the sum of positive-definite terms, so that the inequality can never be satisfied. This already shows the non-existence of closed timelike curves. Furthermore, the only possibility for the equality to be satisfied is whenever for all $i$,

$$
\left.\frac{d z^{\prime}}{d \lambda}\right|_{\lambda^{*}}=\left.\frac{d x^{i}}{d \lambda}\right|_{\lambda^{*}}=0,
$$

which violates the definition of $\lambda$ being an affine parameter. We thus conclude that no closed lightlike curves are allowed in this spacetime.

The corresponding proof for the action generated by $\xi=\partial_{+}+b \partial_{z}$ involves similar ideas and techniques. In this case, the requirement of having an everywhere spacelike Killing vector gives rise to the condition

$$
V(r)^{2 / 3} b^{2}>0 \Rightarrow|b|>0 .
$$

By a linear transformation, we can move to an adapted coordinate system $\left\{z^{\prime}, x^{+\prime}\right\}$ in which the Killing vector becomes a single translation $\xi=\partial_{z^{\prime}}$ and the eleven-dimensional metric takes the form

$$
\begin{aligned}
g=2 V^{-1 / 3} d x^{-}\left(d z^{\prime}+b^{-1} d x^{+\prime}\right)+V^{2 / 3} b^{2}\left(d z^{\prime}\right)^{2} & \\
& +V^{2 / 3} d s^{2}\left(\mathbb{E}^{4}\right)+V^{-1 / 3} d s^{2}\left(\mathbb{E}^{4}\right)^{2} .
\end{aligned}
$$

If we again assume the existence of a closed causal curve of affine parameter $\lambda$ joining the points $\left(x_{0}^{+\prime}, x_{0}^{-}, x_{0}^{i}, z_{0}^{\prime}\right)$ and $\left(x_{0}^{+\prime}, x_{0}^{-}, x_{0}^{i}, z_{0}^{\prime}+\Delta\right)$, there must necessarily exist at least one value for this affine parameter $\lambda^{*}$ where

$$
\exists \lambda^{*} \text { such that }\left.\frac{d x^{-}}{d \lambda}\right|_{\lambda^{*}}=0 .
$$

By computing the norm of the tangent vector to the causal curve at the point $\lambda^{*}$, and using the fact that $|b|>0$, it is immediate to show the non-existence of such closed causal curves by the same argument used before. 


\subsubsection{Supersymmetry}

The determination of the supersymmetric locus can be read off from the results of the M5-brane. We will not repeat the arguments simply state the results, which are contained in Table 9.

\begin{tabular}{|c|c|c|c|}
\hline Translation & Subalgebra & $\nu$ & $\operatorname{dim}$ \\
\hline \hline & $\mathfrak{s u}(4)$ & $\frac{1}{16}$ & 4 \\
$a \partial_{1}+b \partial_{z}$ & $\mathfrak{s p}(1) \times \mathfrak{s p}(1)$ & $\frac{1}{8}$ & 3 \\
& $\mathfrak{s u}(3)$ & $\frac{1}{8}\left(\frac{1}{16}\right)$ & $3(3)$ \\
$a, b$ not both 0 & $\mathfrak{s u}(2)$ & $\frac{1}{4}\left(\frac{1}{8}\right)$ & $2(2)$ \\
& $\{0\}$ & $\frac{1}{2}\left(\frac{1}{4}\right)$ & $1(1)$ \\
\hline & $\mathfrak{s u}(4)$ & $\frac{1}{16}$ & 3 \\
$\partial_{+}+b \partial_{z}$ & $\mathfrak{s p}(1) \times \mathfrak{s p}(1)$ & $\frac{1}{8}$ & 2 \\
& $\mathfrak{s u}(3)$ & $\frac{1}{8}$ & 2 \\
$b \neq 0$ & $\mathfrak{s u}(2)$ & $\frac{1}{4}$ & 1 \\
& $\{0\}$ & $\frac{1}{2}$ & 0 \\
\hline & $\mathfrak{s u}(4)$ & $\frac{1}{16}$ & 4 \\
$a \partial_{0}+b \partial_{z}$ & $\mathfrak{s p}(1) \times \mathfrak{s p}(1)$ & $\frac{1}{8}$ & 3 \\
$a, b$ not both 0 & $\mathfrak{s u}(3)$ & $\frac{1}{8}$ & 3 \\
$|a|<\mu$ & $\mathfrak{s u}(2)$ & $\frac{1}{4}$ & 2 \\
& $\{0\}$ & $\frac{1}{2}$ & 1 \\
\hline
\end{tabular}

Table 9: Supersymmetric reductions of the delocalised M5-brane. We indicate the form of the translation, the spinor isotropy subalgebra to which the rotation belongs, the fraction $\nu$ of the supersymmetry preserved and the dimension of the corresponding stratum of the moduli space $\mathcal{M}$ of supersymmetric reductions. The numbers in parentheses indicate the values in the presence of a null rotation. 


\subsubsection{Explicit reductions}

Let us start the discussion on the explicit configurations by concentrating on the region of the moduli space involving an extra spacelike translation in addition to the one on the delocalised transverse direction $z$. In other words, we shall start by reducing along the orbits of the Killing vector $\xi=\partial_{z}+\alpha$, where

$$
\alpha=a \partial_{1}+\beta B_{02}+\theta_{1} R_{23}+\theta_{2} R_{45}+\theta_{3} R_{67}+\theta_{4} R_{89} .
$$

Notice that by parametrising the reduction in this way, we will be able to discuss both the possibility of fluxbranes and nullbranes at the same time.

The constant matrix $B$ is now $10 \times 10$. In the basis $\left\{x^{0}, x^{1}, \ldots, x^{9}\right\}$, it can be written as

$$
B=\left(\begin{array}{cccccccccc}
0 & 0 & \beta & 0 & 0 & 0 & 0 & 0 & 0 & 0 \\
0 & 0 & 0 & 0 & 0 & 0 & 0 & 0 & 0 & 0 \\
\beta & 0 & 0 & -\theta_{1} & 0 & 0 & 0 & 0 & 0 & 0 \\
0 & 0 & \theta_{1} & 0 & 0 & 0 & 0 & 0 & 0 & 0 \\
0 & 0 & 0 & 0 & 0 & -\theta_{2} & 0 & 0 & 0 & 0 \\
0 & 0 & 0 & 0 & \theta_{2} & 0 & 0 & 0 & 0 & 0 \\
0 & 0 & 0 & 0 & 0 & 0 & 0 & -\theta_{3} & 0 & 0 \\
0 & 0 & 0 & 0 & 0 & 0 & \theta_{3} & 0 & 0 & 0 \\
0 & 0 & 0 & 0 & 0 & 0 & 0 & 0 & 0 & -\theta_{4} \\
0 & 0 & 0 & 0 & 0 & 0 & 0 & 0 & \theta_{4} & 0
\end{array}\right) .
$$

Whenever the extra spacelike translation is non-vanishing $(a \neq 0)$, there will be a non-vanishing 10-vector $\boldsymbol{C}$ taking care of the inhomogeneous part of the symmetry transformation. In the same basis as the one used in the matrix (4.19), this vector is

$$
(C)^{t}=(0, a, \overrightarrow{0})
$$

The corresponding type IIA configurations have a ten-dimensional metric given by

$$
\begin{aligned}
g= & \tilde{\Lambda}^{1 / 2}\left\{d s^{2}\left(\mathbb{E}^{1,5}\right)+V d s^{2}\left(\mathbb{E}^{4}\right)\right\} \\
& -\tilde{\Lambda}^{-1 / 2} V\left\{V^{-1}\left[a d x^{1}+\beta \omega^{02}+\theta_{1} \omega^{23}+\theta_{2} \omega^{45}\right]+\theta_{3} \omega^{67}+\theta_{4} \omega^{89}\right\}^{2}
\end{aligned}
$$

whereas the RR 1-form $A_{1}$, NS-NS 7-form field strength $H_{7}, \mathrm{RR}$ 6-form $H_{6}$ 
and dilaton $\Phi$ are listed below,

$$
\begin{aligned}
A_{1} & =\tilde{\Lambda}^{-1}\left\{V^{-1}\left[a d x^{1}+\beta \omega^{02}+\theta_{1} \omega^{23}+\theta_{2} \omega^{45}\right]+\theta_{3} \omega^{67}+\theta_{4} \omega^{89}\right\} \\
H_{7} & =\operatorname{dvol}\left(\mathbb{E}^{1,5}\right) \wedge d V^{-1} \\
H_{6} & =-\beta\left(x^{2} d x^{2}-x^{0} d x^{0}\right) \wedge d x^{1} \wedge d x^{3} \wedge d x^{4} \wedge d x^{5} \wedge d V^{-1} \\
& -a d x^{0} \wedge d x^{2} \wedge d x^{3} \wedge d x^{4} \wedge d x^{5} \wedge d V^{-1} \\
& -\theta_{1} d x^{0} \wedge d x^{1} \wedge\left(x^{2} d x^{2}+x^{3} d x^{3}\right) \wedge d x^{4} \wedge d x^{5} \wedge d V^{-1} \\
& -\theta_{2} d x^{0} \wedge d x^{1} \wedge d x^{2} \wedge d x^{3} \wedge\left(x^{4} d x^{4}+x^{5} d x^{5}\right) \wedge d V^{-1} \\
\Phi & =\frac{3}{4} \log \left(\tilde{\Lambda} \cdot V^{2 / 3}\right) .
\end{aligned}
$$

The configuration depends on an scalar function $\tilde{\Lambda}$ which is defined in terms of the scalar function $\Lambda$ appearing in the general discussion section, by

$$
\Lambda=V^{2 / 3} \cdot \tilde{\Lambda}
$$

and equals

$$
\begin{aligned}
\tilde{\Lambda}=1+V^{-1}\left\{a^{2}+\left(x^{2}\right)^{2}\left(\theta_{1}^{2}-\beta^{2}\right)+\right. & \left.\left(\beta x^{0}-\theta_{1} x^{3}\right)^{2}\right\}+\left(\theta_{2}\right)^{2}\left[\left(x^{3}\right)^{2}+\left(x^{4}\right)^{2}\right] \\
& +\left(\theta_{3}\right)^{2}\left[\left(x^{6}\right)^{2}+\left(x^{7}\right)^{2}\right]+\left(\theta_{4}\right)^{2}\left[\left(x^{8}\right)^{2}+\left(x^{9}\right)^{2}\right] .
\end{aligned}
$$

We shall discuss the interpretation of the different solutions proceeding in an analogous way to the one followed for the M2-brane Kaluza-Klein reductions. Thus, let us start by examining the subspace of the moduli space of reductions defined by $a=0$. We already know that whenever $\left|\theta_{1}\right|<|\beta|$, there is always a Lorentz observer who sees a pure boost. Such spacetime breaks supersymmetry and contains closed timelike curves. Restricting ourselves to regions of spacetime where such closed causal curves do not exist, their interpretation would give rise to similar cosmological models to the ones discussed in $[45,46,48,49]$. On the other hand, whenever $\left|\theta_{1}\right|>|\beta|$, there is always an observer who measures a pure rotation, so that case would be related to fluxbranes.

After this brief comment, let us study the subset defined by $\beta=0$, that is, the one involving no nullbranes. There are five different possibilities to be considered:

(1) If all $\theta_{i}=0$ we have the well-known NS5-brane in type IIA preserving $\nu=1 / 2$ of the spacetime supersymmetry.

(2) If one of the $\theta$ s is non-vanishing, the configuration describes a composite state involving an NS5-brane and an F7-brane. Depending on the 
chosen $\theta$, its location is a (456789 দ)-plane at $x^{3}=x^{4}=0$ (if $\theta_{1} \neq 0$ ) or a (234589 )-plane at $x^{6}=x^{7}=0$ (if $\theta_{2} \neq 0$ ). In either case, supersymmetry is completely broken due to the presence of these F7-branes.

(3) If there are two non-vanishing $\theta \mathrm{s}$, we must distinguish between four cases:

(i) If $\theta_{1}=\eta_{2} \theta_{2}$, the configuration describes a NS5-brane in the (12345)-plane and an F5-brane along the (16789)-plane sitting at $x^{2}=x^{3}=x^{4}=x^{5}=0$. It preserves $\nu=1 / 4$ of the spacetime supersymmetry, with Killing spinors being preserved by an $\mathfrak{s u}(2)$ subalgebra.

(ii) If $\theta_{3}=\eta_{4} \theta_{4}$, the configuration describes an NS5-brane in the (12345)-plane and an F5-brane along the (12345)-plane sitting at $x^{6}=x^{7}=x^{8}=x^{9}=0$. It preserves $\nu=1 / 4$ of the spacetime supersymmetry, with Killing spinors being preserved by an $\mathfrak{s u}(2)$ subalgebra.

(iii) If $\theta_{1}=\eta_{3} \theta_{3}$, the configuration describes an NS5-brane in the (12345)-plane and an F5-brane along the (14589)-plane sitting at $x^{2}=x^{3}=x^{6}=x^{7}=0$. It preserves $\nu=1 / 4$ of the spacetime supersymmetry, with Killing spinors again preserved by an $\mathfrak{s u}(2)$ subalgebra.

(iv) If $\theta_{i} \neq \eta_{j} \theta_{j}$ for distinct $i, j$, the configuration describes a system of two intersecting F7-branes besides the aforementioned NS5brane. It breaks supersymmetry completely.

(4) If there are three non-vanishing $\theta$ s, we must distinguish among three cases:

(i) If $\theta_{1}=\eta_{2} \theta_{2}+\eta_{3} \theta_{3}$, the configuration describes an NS5-brane in the (12345)-plane and an F3-brane along the (189)-plane sitting at $x^{k}=0 k=2, \ldots, 7$. It preserves $\nu=1 / 8$ of the spacetime supersymmetry, with Killing spinors being preserved by an $\mathfrak{s u}(3)$ subalgebra.

(ii) $\theta_{2}=\eta_{3} \theta_{3}+\eta_{4} \theta_{4}$. In this case, the F3-brane extends along the (123)-plane sitting at $x^{k}=0 k=4, \ldots, 9$. It preserves the same amount of supersymmetry as the previous one due to the existence of an $\mathfrak{s u}(3)$ subalgebra preserving some Killing spinors.

(iii) If $\theta_{i} \neq \eta_{\jmath} \theta_{j}+\eta_{k} \theta_{k}$ for distinct $i, j, k$, the configuration describes the intersection of three different F7-branes plus a NS5-brane. Spacetime supersymmetry is completely broken. 
(5) Finally, in the case of four non-vanishing $\theta$ s, there are generically two inequivalent ways of preserving Killing spinors when four of our deformation parameters are non-vanishing, but due to the symmetries of our configuration, these split into four:

(i) If $\theta_{1}=\eta_{2} \theta_{2}+\eta_{3} \theta_{3}+\eta_{4} \theta_{4}$, the configuration describes an NS5brane in the (12345)-plane and a fluxstring along the $x^{1}$ direction, preserving $\nu=1 / 16$ of the spacetime supersymmetry. This is the one associated with the $\mathfrak{s u}(4)$ isotropy algebra discussed before.

(ii) If $\theta_{1}=\eta_{2} \theta_{2}$ and $\theta_{3}=\eta_{4} \theta_{4}$, the configuration involves an NS5brane and a $\frac{1}{4}$-BPS fluxstring in the $x^{1}$ direction. It preserves $\nu=1 / 8$ and it is associated with the $\mathfrak{s p}(1) \times \mathfrak{s p}(1)$ isotropy algebra.

(iii) If $\theta_{1}=\eta_{3} \theta_{3}$ and $\theta_{2}=\eta_{4} \theta_{4}$, we have the same interpretation as the previous one, but the $\mathfrak{s p}(1) \times \mathfrak{s p}(1)$ isotropy algebra is selected in a different way.

(iv) If all other cases, there are four intersecting F7-branes and a stack of coincident NS5-branes breaking spacetime supersymmetry completely.

If $\beta \neq 0$, the only allowed possibility preserving supersymmetry requires $\left|\theta_{1}\right|=|\beta|$, which is the nullbrane sector. Depending on whether the remaining parameters vanish or satisfy certain linear relations, we distinguish between the following configurations:

(1) If $\theta_{i}=0$ for $i=2,3,4$, the configuration describes an NS5-brane in the (12345)-plane and a nullbrane. This composite configuration preserves $\nu=1 / 4$ of the spacetime supersymmetry.

(2) If one of the $\theta \mathrm{s}$ is non-vanishing, the corresponding configuration breaks supersymmetry completely, due to the presence of an F7-brane besides the previous NS5/nullbrane pair.

(3) If two of the $\theta$ s are non-vanishing, we shall distinguish between three cases:

(i) If $\theta_{i} \neq \eta_{j} \theta_{j}$, for $i \neq j$, the configuration describes a system of two intersecting F7-branes (besides the composite system of a NS5brane and a nullbrane), such that all supersymmetry is broken.

(ii) If $\theta_{2}=\eta_{3} \theta_{3}$, the intersection among the $\mathrm{F} 7$-branes gives rise to the so-called F5-brane, extending along the (12389)-plane and sitting at $x^{4}=x^{5}=x^{6}=x^{7}=0$. The full configuration includes the previous pair NS5/nullbrane system, thus preserving $\nu=1 / 8$. 
(iii) If $\theta_{3}=\eta_{4} \theta_{4}$, one finds a second F5-brane, this time extending along the (12345)-plane and sitting at $x^{6}=x^{7}=x^{8}=x^{9}=0$. As before, the configuration preserves $\nu=1 / 8$.

(4) If all $\theta$ s are non-vanishing, we need to distinguish between two cases:

(i) If $\theta_{2} \neq \eta_{3} \theta_{3}+\eta_{4} \theta_{4}$, the configuration describes the intersection of three F7-branes, in addition to the NS5/nullbrane system, thus breaking all supersymmetry.

(ii) If $\theta_{2}=\eta_{3} \theta_{3}+\eta_{4} \theta_{4}$, the intersection of the F7-branes gives rise to an F3-brane extending along the (123)-plane and sitting at $x^{k}=0$ $\mathrm{k}=4, \ldots, 9$. Thus, there exists a composite configuration involving a NS5-brane, nullbrane and F3-brane preserving $\nu=1 / 16$.

The set of supersymmetric configurations described above is summarised in Table 10, where a similar notation to the one introduced for the M5-brane Kaluza-Klein reductions has been used.

\begin{tabular}{|c|c|l|}
\hline$\nu$ & Object & \multicolumn{1}{|c|}{ Subalgebra } \\
\hline \hline & $\mathrm{NS} 5 \perp \mathrm{F} 5(1)$ & \\
$\frac{1}{4}$ & $\mathrm{NS} 5 \perp \mathrm{F} 5(3)$ & $\mathfrak{s u}(2)$ \\
& $\mathrm{NS} 5 \perp \mathrm{F} 5(5)$ & \\
$\frac{1}{4}$ & $\mathrm{NS} 5+\mathrm{N}$ & $\mathbb{R}$ \\
$\frac{1}{8}$ & $\mathrm{NS} 5 \perp \mathrm{F} 3(1)$ & $\mathfrak{s u}(3)$ \\
& $\mathrm{NS} 5 \perp \mathrm{F} 3(3)$ & \\
$\frac{1}{8}$ & $(\mathrm{NS} 5 \perp \mathrm{F} 5(3))+\mathrm{N}$ & $\mathfrak{s u}(2) \times \mathbb{R}$ \\
& $(\mathrm{NS} 5 \perp \mathrm{F} 5(5))+\mathrm{N}$ & \\
$\frac{1}{8}$ & $\mathrm{NS} 5 \perp \mathrm{F} 1(1)$ & $\mathfrak{s p}(1) \times \mathfrak{s p}(1)$ \\
$\frac{1}{16}$ & $\mathrm{NS} 5 \perp \mathrm{F} 1(1)$ & $\mathfrak{s u}(4)$ \\
$\frac{1}{16}$ & $(\mathrm{NS} 5 \perp \mathrm{F} 3(3))+\mathrm{N}$ & $\mathfrak{s u}(3) \times \mathbb{R}$ \\
\hline
\end{tabular}

Table 10: Supersymmetric configurations of NS5-branes (NS5), fluxbranes and nullbranes.

Before finishing the discussion of the $a=0$ sector, we would like to compute the fluxes associated with F5-branes in the presence of NS5-branes. There are three cases to be considered separately, as indicated in Table 10, and discussed in the text above. Whenever the NS5-branes share a single direction with the F5-brane, $\theta_{3}=\theta_{4}=\beta=0, \theta \equiv \theta_{1}=\theta_{2}$, the RR 1-form 
potential in (4.21) depends on the distance to the origin of the F5-brane plane through the harmonic function $V(r)$. It can be shown that the flux carried by the F5-brane equals the one on flat spacetime, except at $r=0$, where it vanishes. On the other extreme, when the NS5-branes are parallel to the F5-branes $\theta_{1}=\theta_{2}=\beta=0, \theta \equiv \theta_{3}=\theta_{4}$, the RR 1-form potential in (4.21) is independent of the worldvolume F5-brane point. Therefore the flux equals the one carried in flat spacetime everywhere. Finally, when they are two relatively transverse dimensions, $\theta_{2}=\theta_{4}=\beta=0, \theta \equiv \theta_{1}=\theta_{3}$, the RR 1-form potential in (4.21) depends on the relative radial distance in the (89)-plane spanned by the F5-brane. By fixing a point on this plane, it can be shown that

$$
F_{2} \wedge F_{2} \propto-2 \frac{\partial\left(r_{2} \tilde{\Lambda}^{-2}\right)}{\partial r_{1}} d r_{1} \wedge d \theta_{1} \wedge d r_{2} \wedge d \theta_{2} .
$$

Its integral over $\mathbb{R}^{4}$ equals the one in flat spacetime everywhere except at the origin of the (89)-plane, where it vanishes.

All previous considerations were restricted to the $a=0$ subspace. Let us move to the subspace where $a \neq 0$. It is useful to set all the rotation parameters $\theta_{i}$ and $\beta$ to zero. In this case, a similar discussion to the one giving rise to a bound state of $\mathrm{D} 2$-branes and delocalised fundamental strings applies here. Indeed, the construction is entirely analogous just differing in the starting eleven-dimensional background, which now is that of a delocalised M5-brane. Thus, following the same arguments, we will interpret this system as a bound state of NS5-branes and delocalised D4-branes, which still preserves one half of the spacetime supersymmetries. This vacuum allows further supersymmetric configurations both in the fluxbrane and nullbrane sectors. Since the detailed discussion of all these possibilities does not give any new insight and follows closely previous classifications, we simply summarise the results in Table 11.

Notice that the previous discussion does not cover the particular case $b=0$ and $a \neq 0$ in 9 . That would give rise to delocalised D4-branes in the presence of fluxbranes, whenever $\theta_{i} \neq 0$. Since we are not particularly interested in the study of delocalised branes in the presence of fluxbranes, we shall not present the details for these configurations.

Let us move to the region of the moduli space where the extra translation is along a null direction. That is, the starting Killing vector is decomposed as $\xi=\partial_{z}+\alpha$, where

$$
\alpha=\partial_{+}+\theta_{1} R_{23}+\theta_{2} R_{45}+\theta_{3} R_{67}+\theta_{4} R_{89} .
$$




\begin{tabular}{|c|c|l|}
\hline$\nu$ & Object & \multicolumn{1}{|c|}{ Subalgebra } \\
\hline \hline$\frac{1}{2}$ & NS5-D4 & $\{0\}$ \\
& $(\mathrm{NS5} 5$-D4) $\perp \mathrm{F} 5(1)$ & \\
$\frac{1}{4}$ & $(\mathrm{NS5}-\mathrm{D} 4) \perp \mathrm{F} 5(3)$ & $\mathfrak{s u}(2)$ \\
& $(\mathrm{NS} 5-\mathrm{D} 4) \perp \mathrm{F} 5(5)$ & \\
$\frac{1}{4}$ & $(\mathrm{NS} 5-\mathrm{D} 4)+\mathrm{N}$ & $\mathbb{R}$ \\
$\frac{1}{8}$ & $(\mathrm{NS} 5-\mathrm{D} 4) \perp \mathrm{F} 3(1)$ & $\mathfrak{s u}(3)$ \\
& $(\mathrm{NS} 5-\mathrm{D} 4) \perp \mathrm{F} 3(3)$ & \\
$\frac{1}{8}$ & $((\mathrm{NS} 5-\mathrm{D} 4) \perp \mathrm{F} 5(3))+\mathrm{N}$ & $\mathfrak{s u}(2) \times \mathbb{R}$ \\
& $((\mathrm{NS} 5-\mathrm{D} 4) \perp \mathrm{F} 5(5))+\mathrm{N}$ & \\
$\frac{1}{8}$ & $(\mathrm{NS} 5-\mathrm{D} 4) \perp \mathrm{F} 1(1)$ & $\mathfrak{s p}(1) \times \mathfrak{s p}(1)$ \\
$\frac{1}{16}$ & $(\mathrm{NS} 5-\mathrm{D} 4) \perp \mathrm{F} 1(1)$ & $\mathfrak{s u}(4)$ \\
$\frac{1}{16}$ & $((\mathrm{NS} 5-\mathrm{D} 4) \perp \mathrm{F} 3(3))+\mathrm{N}$ & $\mathfrak{s u}(3) \times \mathbb{R}$ \\
\hline
\end{tabular}

Table 11: Supersymmetric configurations of bound states made of NS5branes and delocalised D4-branes (NS5-D4), fluxbranes and nullbranes.

The constant matrix $B$ is a $9 \times 9$ one, leaving the second null direction $x^{-}$ invariant. In the basis $\left\{x^{+}, x^{2}, \ldots, x^{9}\right\}$, it can be written as

$$
B=\left(\begin{array}{ccccccccc}
0 & 0 & 0 & 0 & 0 & 0 & 0 & 0 & 0 \\
0 & 0 & -\theta_{1} & 0 & 0 & 0 & 0 & 0 & 0 \\
0 & \theta_{1} & 0 & 0 & 0 & 0 & 0 & 0 & 0 \\
0 & 0 & 0 & 0 & -\theta_{2} & 0 & 0 & 0 & 0 \\
0 & 0 & 0 & \theta_{2} & 0 & 0 & 0 & 0 & 0 \\
0 & 0 & 0 & 0 & 0 & 0 & -\theta_{3} & 0 & 0 \\
0 & 0 & 0 & 0 & 0 & \theta_{3} & 0 & 0 & 0 \\
0 & 0 & 0 & 0 & 0 & 0 & 0 & 0 & -\theta_{4} \\
0 & 0 & 0 & 0 & 0 & 0 & 0 & \theta_{4} & 0
\end{array}\right) .
$$

Since there is an extra null translation (its parameter can always be set to one), there is a non-vanishing 9-vector $\boldsymbol{C}$ taking care of the inhomogeneous part of the isometry transformation. In the same basis as the one used in the matrix (4.22), this vector is

$$
(C)^{t}=(1, \overrightarrow{0})
$$

The corresponding type IIA configurations have a ten-dimensional metric 
given by

$$
\begin{aligned}
g=\tilde{\Lambda}^{1 / 2}\{ & \left.d s^{2}\left(\mathbb{E}^{1,5}\right)+V d s^{2}\left(\mathbb{E}^{4}\right)\right\} \\
& -\tilde{\Lambda}^{-1 / 2} V\left\{\theta_{3} \omega^{67}+\theta_{4} \omega^{89}+V^{-1}\left[d x^{-}+\theta_{1} \omega^{23}+\theta_{2} \omega^{45}\right]\right\}^{2},
\end{aligned}
$$

whereas the RR 1-form $A_{1}$, NS-NS 7-form field strength $H_{7}$, RR 6-form $H_{6}$ and dilaton $\Phi$ are listed below

$$
\begin{aligned}
A_{1} & =\tilde{\Lambda}^{-1}\left\{V^{-1}\left[d x^{-}+\theta_{1} \omega^{23}+\theta_{2} \omega^{45}\right]+\theta_{3} \omega^{67}+\theta_{4} \omega^{89}\right\} \\
H_{7} & =\operatorname{dvol}\left(\mathbb{E}^{1,5}\right) \wedge d V^{-1} \\
H_{6} & =d x^{-} \wedge d \operatorname{dvol}\left(\mathbb{E}^{4}\right) \wedge d V^{-1} \\
& -\theta_{1} d x^{+} \wedge d x^{-} \wedge\left(x^{2} d x^{2}+x^{3} d x^{3}\right) \wedge d x^{4} \wedge d x^{5} \wedge d V^{-1} \\
& -\theta_{2} d x^{+} \wedge d x^{-} \wedge d x^{2} \wedge d x^{3} \wedge\left(x^{4} d x^{4}+x^{5} d x^{5}\right) \wedge d V^{-1} \\
\Phi & =\frac{3}{4} \log \left(\tilde{\Lambda} \cdot V^{2 / 3}\right) .
\end{aligned}
$$

The configuration depends on an scalar function $\tilde{\Lambda}$ which is defined in terms of the scalar function $\Lambda$ appearing in the general discussion section, by

$$
\Lambda=V^{2 / 3} \cdot \tilde{\Lambda}
$$

and equals

$$
\begin{aligned}
\tilde{\Lambda}=1+V^{-1}\left\{\left(\theta_{1}\right)^{2}\left[\left(x^{2}\right)^{2}+\left(x^{3}\right)^{2}\right]\right. & \left.+\left(\theta_{2}\right)^{2}\left[\left(x^{4}\right)^{2}+\left(x^{5}\right)^{2}\right]\right\} \\
& +\left(\theta_{3}\right)^{2}\left[\left(x^{6}\right)^{2}+\left(x^{7}\right)^{2}\right]+\left(\theta_{4}\right)^{2}\left[\left(x^{8}\right)^{2}+\left(x^{9}\right)^{2}\right] .
\end{aligned}
$$

Whenever all $\theta$ s vanish, and following a similar discussion to the one presented when dealing with a delocalised M2-brane, one should expect to get a configuration which interpolates among a wave background at asymptotic infinity and a linear dilaton background [83], which is the corresponding geometry close to a stack of NS5-branes. This is indeed straightforward to check. The stability and supersymmetry of the configuration require both $H_{6}$ and $F_{2}=d A_{1}$ to be null forms, but its role is not clear to us. Switching on the $\theta_{i}$ parameters, one is adding fluxbranes to the previous configuration.

Let us finally move to the region of the moduli space where the extra translation is along a time direction. That is, the starting Killing vector is decomposed as $\xi=\partial_{z}+\alpha$, where

$$
\alpha=a \partial_{0}+\theta_{1} R_{23}+\theta_{2} R_{45}+\theta_{3} R_{67}+\theta_{4} R_{89} .
$$

The constant matrix $B$ is now $9 \times 9$, leaving the spacelike direction $x^{1}$ invariant. Formally, it is given by the matrix (4.22), but this time written in 
the basis $\left\{x^{0}, x^{2}, \ldots, x^{9}\right\}$. The inhomogeneous part of the isometry transformation defines a non-trivial 9-vector $\boldsymbol{C}$

$$
(C)^{t}=(a, \overrightarrow{0}) .
$$

The corresponding type IIA configurations have a ten-dimensional metric given by,

$$
\begin{aligned}
g=\tilde{\Lambda}^{1 / 2} & \left\{d s^{2}\left(\mathbb{E}^{1,5}\right)+V d s^{2}\left(\mathbb{E}^{4}\right)\right\} \\
& -\tilde{\Lambda}^{-1 / 2} V\left\{\theta_{3} \omega^{67}+\theta_{4} \omega^{89}+V^{-1}\left[-a d x^{0}+\theta_{1} \omega^{23}+\theta_{2} \omega^{45}\right]\right\}^{2},
\end{aligned}
$$

where we remind the reader that $\omega^{i j}:=x^{i} d x^{j}-x^{\jmath} d x^{i}$. In addition the RR 1-form $A_{1}$, NS-NS 7 -form field strength $H_{7}, \mathrm{RR} 6$-form $H_{6}$ and dilaton $\Phi$ are listed below

$$
\begin{aligned}
A_{1} & =\tilde{\Lambda}^{-1}\left\{V^{-1}\left[-a d x^{0}+\theta_{1} \omega^{23}+\theta_{2} \omega^{45}\right]+\theta_{3} \omega^{67}+\theta_{4} \omega^{89}\right\} \\
H_{7} & =\operatorname{dvol}\left(\mathbb{E}^{1,5}\right) \wedge d V^{-1} \\
H_{6} & =a d x^{0} \wedge \operatorname{dvol}\left(\mathbb{E}^{5}\right) \wedge d V^{-1} \\
& -\theta_{1} d x^{0} \wedge d x^{1} \wedge\left(x^{2} d x^{2}+x^{3} d x^{3}\right) \wedge d x^{4} \wedge d x^{5} \wedge d V^{-1} \\
& -\theta_{2} d x^{0} \wedge d x^{1} \wedge d x^{2} \wedge d x^{3} \wedge\left(x^{4} d x^{4}+x^{5} d x^{5}\right) \wedge d V^{-1} \\
\Phi & =\frac{3}{4} \log \left(\tilde{\Lambda} \cdot V^{2 / 3}\right) .
\end{aligned}
$$

The configuration depends on an scalar function $\tilde{\Lambda}$ which is defined in terms of the scalar function $\Lambda$ appearing in the general discussion section, by

$$
\Lambda=V^{2 / 3} \cdot \tilde{\Lambda}
$$

and equals

$$
\begin{array}{r}
\tilde{\Lambda}=1+V^{-1}\left\{-a^{2}+\left(\theta_{1}\right)^{2}\left[\left(x^{2}\right)^{2}+\left(x^{3}\right)^{2}\right]+\left(\theta_{2}\right)^{2}\left[\left(x^{4}\right)^{2}+\left(x^{5}\right)^{2}\right]\right\} \\
+\left(\theta_{3}\right)^{2}\left[\left(x^{6}\right)^{2}+\left(x^{7}\right)^{2}\right]+\left(\theta_{4}\right)^{2}\left[\left(x^{8}\right)^{2}+\left(x^{9}\right)^{2}\right] .
\end{array}
$$

We shall follow the same strategy as for the similar construction regarding the M2-brane. If we set $\theta_{i}=0 \forall \mathrm{i}$, we are left with a ten-dimensional configuration whose geometry is given by

$$
\begin{aligned}
g=-\left(V-a^{2}\right)^{-1 / 2} V^{1 / 2} & \left(d x^{0}\right)^{2} \\
+ & \left(V-a^{2}\right)^{1 / 2}\left\{V^{-1 / 2} d s^{2}\left(\mathbb{E}^{5}\right)+V^{1 / 2} d s^{2}\left(\mathbb{E}^{4}\right)\right\} .
\end{aligned}
$$


As before, the condition $|a|<1$ ensures the absence of horizons in spacetime. If we would have allowed $|a| \geq 1$, there would have been horizons at

$$
r_{\mathrm{H}}^{2}=\frac{Q}{a^{2}-1},
$$

dividing spacetime into regions $\left(r>r_{\mathrm{H}}\right)$ having closed timelike curves and regions $\left(r<r_{\mathrm{H}}\right)$ free of these causal singularities.

We shall not add any further comments regarding the physical interpretation of these configurations, besides the possibility of looking at them as bound states of NS5-branes and delocalised E4-branes [79, 80, 81]. They are again interpolating among flat spacetime and the linear dilaton background [83]. It is straightforward to add fluxbranes by switching on the $\theta_{i}$ parameters, while still preserving some supersymmetry.

Looking at table 9, we learn that the previous Kaluza-Klein reduction does not cover the case $b=0$ and $a \neq 0$, which is certainly allowed if both $\theta_{3}$ and $\theta_{4}$ are non-vanishing. We include the corresponding ten dimensional configuration below for completeness, even though its physical interpretation is unclear to us and the final background is delocalised in the $z$ direction. The type IIA metric is given by

$$
\begin{aligned}
g=V^{-1 / 2} \tilde{\Lambda}^{1 / 2} d s^{2}\left(\mathbb{E}^{5}\right)+V^{1 / 2} \tilde{\Lambda}^{1 / 2}\left[(d z)^{2}+d s^{2}\left(\mathbb{E}^{4}\right)\right] \\
-V^{-1 / 2} \tilde{\Lambda}^{-1 / 2}\left[\theta_{1} \omega^{23}+\theta_{2} \omega^{45}+V\left(\theta_{3} \omega^{67}+\theta_{4} \omega^{89}\right)\right]^{2}
\end{aligned}
$$

where we remind the reader that $\omega^{i j}:=x^{i} d x^{j}-x^{j} d x^{i}$. In addition the RR 1-form $A_{1}$, RR 6-form $H_{6}$ and dilaton $\Phi$ are non-trivial and listed below

$$
\begin{aligned}
A_{1} & =\tilde{\Lambda}^{-1}\left\{\theta_{1} \omega^{23}+\theta_{2} \omega^{45}+V\left(\theta_{3} \omega^{67}+\theta_{4} \omega^{89}\right)\right\} \\
H_{6} & =-a \operatorname{dvol}\left(\mathbb{E}^{5}\right) \wedge d V^{-1} \\
\Phi & =\frac{3}{4} \log \left(\tilde{\Lambda} \cdot V^{-1 / 3}\right) .
\end{aligned}
$$

The configuration depends on an scalar function $\tilde{\Lambda}$ which is defined in terms of the scalar function $\Lambda$ appearing in the general discussion section, by

$$
\Lambda=V^{-1 / 3} \cdot \tilde{\Lambda}
$$

and equals

$$
\begin{aligned}
\tilde{\Lambda}=-a^{2}+\left(\theta_{1}\right)^{2}\left[\left(x^{2}\right)^{2}+\left(x^{3}\right)^{2}\right] & +\left(\theta_{2}\right)^{2}\left[\left(x^{4}\right)^{2}+\left(x^{5}\right)^{2}\right] \\
& +V\left\{\left(\theta_{3}\right)^{2}\left[\left(x^{6}\right)^{2}+\left(x^{7}\right)^{2}\right]+\left(\theta_{4}\right)^{2}\left[\left(x^{8}\right)^{2}+\left(x^{9}\right)^{2}\right]\right\} .
\end{aligned}
$$




\section{IIA/IIB discrete quotients and duality}

All the ideas and formalism developed so far apply equally well to any background in type IIA/IIB supergravity having some isometry group $G$. In particular, D-brane backgrounds [84] allow an analogous description of the form (1.7) and adding the corresponding non-trivial profile for the dilaton. Their Killing spinors satisfy the same properties as the ones discussed in Section 1.4. Thus we can again conclude that the action of the symmetry group on the Killing spinors is induced by the action of $\operatorname{Spin}(1, p) \times \operatorname{Spin}(9-p)$ on the asymptotic spinors. Once more, translations will act trivially on spinors, whereas Lorentz transformations will impose certain constraints analogous to the vanishing of (1.9). It should conceptually be clear, that we could classify the (not necessarily) supersymmetric freely-acting spacelike isometries as we did for the M2-brane and M5-brane configurations. By constructing the discrete quotients $\mathcal{M}_{\mathrm{Dp}} / \Gamma_{0}, \Gamma_{0}$ being some discrete subgroup of the corresponding one-parameter subgroup $\Gamma$, such that $\Gamma / \Gamma_{0}$ is compact, we would reach new supersymmetric smooth type IIA/IIB configurations.

It is nevertheless well-known that the configurations described in the previous sections are dual to the ones outlined above. Consider an M-theory background reduced along the orbits of $\xi_{1}=\partial_{z}+\lambda$, with a further compact spacelike direction $x$, such that translations along it, infinitesimally generated by $\xi_{2}=\partial_{x}$, commute with the action generated by $\xi_{1}$. The claim is that the type IIA configuration obtained through Kaluza-Klein reduction along the orbits of $\xi_{1}$ is equivalent to the one obtained through KaluzaKlein reduction along the orbits of $\xi_{2}$, applying a T-duality transformation [78] along the orbits of $\xi_{1}$, plus an S-duality transformation in type IIB, and finally, after a relabelling of coordinates, applying a T-duality back to type IIA. The mechanism just described is an obvious extension of the well-known M-theory flip, when one of the orbits is twisted.

After all these preliminary remarks, it should be clear that most of the results derived previously extend straightforwardly for $\mathrm{D} p$-branes, among other configurations in type IIA/IIB. In particular, it should be clear that we can construct time-dependent backgrounds starting from $\mathrm{D} p$-branes $(p \geq 3)$ and constructing the quotient manifold associated with the discrete identification giving rise to the ten-dimensional nullbrane. Similar comments would apply for the fluxbrane sector. As a particular example, let us consider D3-branes. We shall concentrate on the non-trivial identifications preserving $\nu=1 / 4$ of the spacetime supersymmetry generated by $\xi=\partial_{z}+\lambda$, where $\partial_{z}$ generates translations along the brane and $\lambda$ is either a rotation $\rho$ belonging to an $\mathfrak{s u}(2)$ subalgebra (flux 5-brane construction) or a null rotation $\nu$ belonging 
to an $\mathbb{R}$ subalgebra (nullbrane construction). Both quotients survive the near horizon limit, even though they break the superconformal symmetries, so that there is no supersymmetry enhancement in this case. It is natural to ask about the corresponding gauge theory dual for type IIB in these configurations, and it is natural to guess that it will be given in terms of the corresponding orbifold constructions in $\mathcal{N}=4$ supersymmetric Yang-Mills.

Let us denote the matter fields transforming in the adjoint representation of $\mathrm{SU}(N)$ by $\phi^{i} i=1, \ldots, 6$, whereas the coordinates of the four-dimensional manifold where the field theory is defined will be denoted by $\left(x^{\mu}, z\right) \mu=$ $0,1,2$ and $z$ standing for the compact one. Due to the isometries of the background, there are two inequivalent $\mathfrak{s u}(2)$ constructions. Indeed, one may consider a rotation $\rho_{\perp}$ acting on the transverse directions to the brane, or a rotation which acts both on $Z_{1}=\phi^{1}+i \phi^{2}$ and $\omega=x^{1}+i x^{2}$. In the first case, the compatibility of the gauge structure of the theory with the orbifold requires the matrices $Z_{1}$ and $Z_{2}=\phi^{3}+i \phi^{4}$ to satisfy

$$
\begin{aligned}
& Z_{1}\left(x^{\mu}, z+R\right)=e^{i \theta} \Omega\left(x^{\mu}, z\right) Z_{1}\left(x^{\mu}, z\right)\left(\Omega\left(x^{\mu}, z\right)\right)^{-1} \\
& Z_{2}\left(x^{\mu}, z+R\right)=e^{-i \theta} \Omega\left(x^{\mu}, z\right) Z_{2}\left(x^{\mu}, z\right)\left(\Omega\left(x^{\mu}, z\right)\right)^{-1}
\end{aligned}
$$

where $\Omega\left(x^{\mu}, z\right)$ is an $\mathrm{SU}(N)$ group element describing a gauge transformation, whereas the remaining two adjoint matrices satisfy the standard ones

$$
\phi^{i}\left(x^{\mu}, z+R\right)=\Omega\left(x^{\mu}, z\right) \phi^{i}\left(x^{\mu}, z\right)\left(\Omega\left(x^{\mu}, z\right)\right)^{-1} \quad i=5,6 .
$$

In the second case, since the group also acts non-trivially on the $\omega$ plane where the field theory is defined, one has four adjoint matrices satisfying

$$
\phi^{i}\left(x^{0}, e^{i \theta} \omega, z+R\right)=\Omega\left(x^{\mu}, z\right) \phi^{i}\left(x^{\mu}, z\right)\left(\Omega\left(x^{\mu}, z\right)\right)^{-1} \quad i=3,4,5,6,
$$

whereas the $Z_{1}$ is twisted by a constant phase

$$
Z_{1}\left(x^{0}, e^{i \theta} \omega, z+R\right)=e^{-i \theta} \Omega\left(x^{\mu}, z\right) Z_{1}\left(x^{\mu}, z\right)\left(\Omega\left(x^{\mu}, z\right)\right)^{-1} .
$$

Finally, in the null rotation identification, all adjoint matrices $\phi^{i}$ satisfy that for all $i$,

$$
\phi^{i}\left(\tilde{x}^{\mu}, z+R\right)=\Omega\left(x^{\mu}, z\right) \phi^{i}\left(x^{\mu}, z\right)\left(\Omega\left(x^{\mu}, z\right)\right)^{-1},
$$

where $\tilde{x}^{\mu}$ stands for the image of $x^{\mu}$ under a (not infinitesimal) null rotation. 


\section{Acknowledgments}

We started this work while participating in the programme Mathematical Aspects of String Theory at the Erwin Schrödinger Institute in Vienna, and it is again a pleasure to thank them for support and for providing such a stimulating environment in which to do research. JMF's participation in this programme was made possible in part by a travel grant from PPARC. The work was finished while JMF was visiting the IHÉS, whom he would like to thank for support. JS would like to thank the School of Mathematics of the University of Edinburgh for hospitality during the final stages of this work. JMF is a member of EDGE, Research Training Network HPRN-CT-2000-00101, supported by The European Human Potential Programme. The research of JMF is partially supported by the EPSRC grant GR/R62694/01. JS is supported by a Marie Curie Fellowship of the European Community programme "Improving the Human Research Potential and the Socio-Economic Knowledge Base" under the contract number HPMF-CT-2000-00480, and in part by a grant from the United States-Israel Binational Science Foundation (BSF), the European Research Training Network HPRN-CT-2000-00122 and by Minerva.

\section{A Group theory and spinors}

In this appendix we collect some facts about how the spinor representation of $\operatorname{Spin}(1,10)$ decomposes under certain subgroups. These results are useful in determining the supersymmetric Kaluza-Klein reductions of the M2 and M5-brane solutions.

Let us start by recalling a few facts about the irreducible representations of $\operatorname{Spin}(1,10)$ and of the Clifford algebra $C \ell(1,10)$. The Clifford algebra $\mathrm{C} \ell(1,10)$ is isomorphic (as a real associative algebra) to $\operatorname{Mat}(32, \mathbb{R}) \oplus$ $\operatorname{Mat}(32, \mathbb{R})$, where $\operatorname{Mat}(n, \mathbb{R})$ is the algebra of $n \times n$ real matrices. This means that there are two inequivalent irreducible representations: real and of dimension 32. They are distinguished by the action of the centre which is generated by the volume form

$$
\operatorname{dvol}\left(\mathbb{E}^{1,10}\right):=\Gamma_{01 \cdots \natural},
$$

which squares to the identity.

The condition (3.4) translates into an eight-dimensional chirality condi- 
tion on the spinor. Indeed, decomposing $\operatorname{dvol}\left(\mathbb{E}^{1,10}\right)$ into a product

$$
\operatorname{dvol}\left(\mathbb{E}^{1,10}\right)=\operatorname{dvol}\left(\mathbb{E}^{1,2}\right) \operatorname{dvol}\left(\mathbb{E}^{8}\right)=\Gamma_{012} \Gamma_{34 \cdots \natural}
$$

of two commuting operators, we obtain

$$
\operatorname{dvol}\left(\mathbb{E}^{1,2}\right) \varepsilon=\operatorname{dvol}\left(\mathbb{E}^{8}\right) \operatorname{dvol}\left(\mathbb{E}^{1,10}\right) \varepsilon= \pm \operatorname{dvol}\left(\mathbb{E}^{8}\right) \varepsilon,
$$

where the sign depends on the action of $\operatorname{dvol}\left(\mathbb{E}^{1,10}\right)$, equivalently on the choice of irreducible representation of $\mathrm{C} \ell(1,10)$. Let us assume that a choice has been made once and for all and let $S_{11}$ denote the corresponding irreducible representation. This is an irreducible representation of $\operatorname{Spin}(1,10)$. Under the natural Spin(10) subgroup, $S_{11}$ remains irreducible as a real representation, even though its complexification is reducible. This is because $\operatorname{dvol}\left(\mathbb{E}^{10}\right)$ is a complex structure and to diagonalise it requires complexifying the spinors. Indeed, we have

$$
S_{11} \otimes \mathbb{C}=S_{10} \oplus \bar{S}_{10}
$$

where $S_{10}$ consists of those complex spinors $\varepsilon$ such that

$$
\operatorname{dvol}\left(\mathbb{E}^{10}\right) \cdot \varepsilon=i \varepsilon,
$$

and $\bar{S}_{10}$ is the complex conjugate. We will abbreviate equation (A.1) with the notation

$$
S_{11}=\llbracket S_{10} \rrbracket .
$$

In other words, the double brackets indicate the underlying real representation of the representation obtained by adding to a complex representation its complex conjugate, which has a natural real structure. Notice that $\operatorname{dim}_{\mathbb{R}} \llbracket S_{10} \rrbracket=2 \operatorname{dim}_{\mathbb{C}} S_{10}$ (which in this case is 32 ), as the notation tries to suggest. We are interested in how $S_{11}$ breaks under the natural $\operatorname{Spin}(2) \times \operatorname{Spin}(8)$ subgroup of $\operatorname{Spin}(10)$. Since the volume element of $\mathbb{E}^{2}$ is a complex structure, whereas that of $\mathbb{E}^{8}$ squares to the identity, we see that

$$
S_{10}=\left(S_{2} \otimes S_{8}^{+}\right) \oplus\left(\bar{S}_{2} \otimes S_{8}^{-}\right),
$$

where $S_{8}^{ \pm}$are the half-spin representations of $\operatorname{Spin}(8)$, and $S_{2}$ is the onedimensional complex irreducible representation of $\operatorname{Spin}(2)$ with weight 1 ; that is, $S_{2}$ is the "half-spin" representation of Spin(2). Since the representations $S_{8}^{ \pm}$are real,

$$
S_{11}=\llbracket S_{2} \rrbracket \otimes\left(S_{8}^{+} \oplus S_{8}^{-}\right),
$$

whence the subspace of $S_{11}$ consisting of spinors which obey (3.4) transforms under $\operatorname{Spin}(2) \times \operatorname{Spin}(8)$ as $\llbracket S_{2} \rrbracket \otimes S_{8}^{ \pm}$, for some choice of sign. 
The problem of determining which Kaluza-Klein reductions of the (delocalised) M2 brane preserve some supersymmetry comes down to determining which elements in (the Lie algebra of a maximal torus of) $\operatorname{Spin}(2) \times \operatorname{Spin}(8)$ (or $\operatorname{Spin}(2) \times \operatorname{Spin}(7)$ for the delocalised M2 brane) preserve a spinor in $\llbracket S_{2} \rrbracket \otimes S_{8}^{ \pm}$. As explained in Section 2.2 , one way to do this is to simply determine the weight decomposition of $\llbracket S_{2} \rrbracket \otimes S_{8}^{ \pm}$under the maximal torus. We will work infinitesimally, hence we will decompose $\llbracket S_{2} \rrbracket \otimes S_{8}^{ \pm}$under a Cartan subalgebra of $\mathfrak{s o}(2) \times \mathfrak{s o}(8)$.

Up to conjugation, a typical element in $\mathfrak{s o}(2) \times \mathfrak{s o}(8)$ can be written as

$$
\theta_{1} R_{12}+\theta_{2} R_{34}+\theta_{3} R_{56}+\theta_{4} R_{78}+\theta_{5} R_{9 \natural}
$$

where the infinitesimal rotations $R_{i j}$ generate a Cartan subalgebra in $\mathfrak{s o}(2) \times$ $\mathfrak{s o}(8)$, with $R_{12}$ spanning $\mathfrak{s o}(2)$ and the rest spanning a Cartan subalgebra of $\mathfrak{s o}(8)$. In the case of the delocalised M2-brane, we must restrict to a $\mathfrak{s o}$ (7) subalgebra, which means setting $\theta_{5}=0$, say. Relative to a basis for the root space canonically dual to the $R_{i j}$, the weights of the representation $\llbracket S_{2} \rrbracket \otimes S_{8}^{ \pm}$are easy to work out. First of all $\llbracket S_{2} \rrbracket$ has weights \pm 1 , whereas $S_{8}^{ \pm}$ has weights $( \pm 1, \pm 1, \pm 1, \pm 1)$ where the signs are uncorrelated, but where their product is \pm 1 for $S_{8}^{ \pm}$respectively. Putting these two results together we find that the weights of $\llbracket S_{2} \rrbracket \otimes S_{8}^{ \pm}$are $( \pm 1, \pm 1, \pm 1, \pm 1, \pm 1)$ where the signs are uncorrelated but where the product of all but the first sign is \pm 1 for $\llbracket S_{2} \rrbracket \otimes S_{8}^{ \pm}$, respectively. In other words, we have

$$
\begin{aligned}
& \text { weights }\left(\llbracket S_{2} \rrbracket \otimes S_{8}^{ \pm}\right)= \\
& \qquad\left\{\left(\mu_{1}, \mu_{2}, \mu_{3}, \mu_{4}, \mu_{5}\right) \mid \mu_{i}^{2}=1 \text { and } \prod_{i=2}^{5} \mu_{i}= \pm 1\right\} .
\end{aligned}
$$

For the (delocalised) M5-brane we have to break $S_{11}$ into irreducible representations of $\operatorname{Spin}(1,5) \times \operatorname{Spin}(5)$ or $\operatorname{Spin}(1,5) \times \operatorname{Spin}(4)$. The situation is analogous. We can decompose the volume element $\operatorname{dvol}\left(\mathbb{E}^{1,10}\right)$ into a product

$$
\operatorname{dvol}\left(\mathbb{E}^{1,10}\right)=\operatorname{dvol}\left(\mathbb{E}^{1,5}\right) \operatorname{dvol}\left(\mathbb{E}^{5}\right)=\Gamma_{01 \ldots 5} \Gamma_{67 \cdots \emptyset}
$$

of commuting operators which both square to the identity. Again a choice for the value of $\operatorname{dvol}\left(\mathbb{E}^{1,10}\right)$ on the representation $S$ translates the condition (4.4) into a five-dimensional chirality condition on the spinor:

$$
\operatorname{dvol}\left(\mathbb{E}^{1,5}\right) \varepsilon=\operatorname{dvol}\left(\mathbb{E}^{5}\right) \operatorname{dvol}\left(\mathbb{E}^{1,10}\right) \varepsilon= \pm \operatorname{dvol}\left(\mathbb{E}^{5}\right) \varepsilon,
$$

where the sign depends on the choice of irreducible representation $S_{11}$. The low-dimensional isomorphisms

$$
\operatorname{Spin}(1,5) \cong \operatorname{SL}(2, H) \quad \text { and } \quad \operatorname{Spin}(5) \cong \operatorname{Sp}(2)
$$


tell us that the irreducible representations of these groups are two-dimensional quaternionic; equivalently, four-dimensional complex with a quaternionic structure. Let $S_{6}^{ \pm}$denote the positive and negative chirality half-spin representations of $\operatorname{Spin}(1,5)$ and let $S_{5}$ denote the half-spin representation of Spin(5). The complexified spinors break up as

$$
S_{11} \otimes \mathbb{C}=\left(S_{6}^{+} \oplus S_{6}^{-}\right) \otimes S_{5} .
$$

The right-hand side also has a natural real structure, since the tensor product of two quaternionic representations is real. We can summarise this relationship by

$$
S_{11}=\left[\left(S_{6}^{+} \oplus S_{6}^{-}\right) \otimes S_{5}\right],
$$

where the single brackets denote the underlying real representation of a complex representation admitting a real structure. (Notice that $\operatorname{dim}_{\mathbb{R}}[V]=$ $\operatorname{dim}_{\mathbb{C}} V$, which agrees with $\operatorname{dim}_{\mathbb{R}} S_{11}=32$ as it should.) The subspace of spinors satisfying (4.4) transforms as $\left[S_{6}^{ \pm} \otimes S_{5}\right]$, where the sign depends on the choice of $S_{11}$. In order to determine which Kaluza-Klein reductions of the (delocalised) M5 brane preserve some supersymmetry, we need to determine the weights decomposition of $\left[S_{6}^{ \pm} \otimes S_{5}\right]$ under a Cartan subalgebra of $\operatorname{Spin}(5) \times \operatorname{Spin}(5)$. Both half-spin representations $S_{6}^{ \pm}$of $\operatorname{Spin}(1,5)$ are isomorphic to $S_{5}$ as representations of $\operatorname{Spin}(5)$. Therefore we are interested in the first instance in the weight decomposition of $S_{5}$ under a Cartan subalgebra of $\operatorname{Spin}(5)$ and then in that of $\left[S_{5} \otimes S_{5}\right]$ under a Cartan subalgebra of $\operatorname{Spin}(5) \times \operatorname{Spin}(5)$. A typical element of the Cartan subalgebra of $\operatorname{Spin}(5)$ can be written as

$$
\theta_{1} R_{12}+\theta_{2} R_{34}
$$

in the same notation as that used above. Relative to a basis for the weight system which is canonically dual to the $R_{i j}$, the weights of the representation $S_{5}$ are given by $\{( \pm 1, \pm 1)\}$ with uncorrelated signs for a total of four weights. Similarly, the weight decomposition of $\left[S_{5} \otimes S_{5}\right]$ is given by

$$
\text { weights }\left(\left[S_{5} \otimes S_{5}\right]\right)=\left\{\left(\mu_{1}, \mu_{2}, \mu_{3}, \mu_{4}\right) \mid \mu_{i}^{2}=1\right\} .
$$

\section{References}

[1] M. Melvin, "Pure magnetic and electric geons," Phys. Lett. 8 (1964) 65-68. 
[2] G. Gibbons and D. Wiltshire, "Spacetime as a membrane in higher dimensions," Nucl. Phys. B287 (1987) 717. arXiv:hep-th/0109093.

[3] G. Gibbons and K. Maeda, "Black holes and membranes in higher-dimensional theories with dilaton fields," Nucl. Phys. B298 (1988) 741-775.

[4] F. Dowker, J. Gauntlett, S. Giddings, and G. Horowitz, "On pair creation of extremal black holes and Kaluza-Klein monopoles," Phys. Rev. D50 (1994) 2662-2679. arXiv:hep-th/9312172.

[5] F. Dowker, J. Gauntlett, G. Gibbons, and G. Horowitz, "The decay of magnetic fields in Kaluza-Klein theory," Phys. Rev. D52 (1995) 6929-6940. arXiv:hep-th/9507143.

[6] F. Dowker, J. Gauntlett, G. Gibbons, and G. Horowitz, "Nucleation of p-branes and fundamental strings," Phys. Rev. D53 (1996) 7115-7128. arXiv:hep-th/9512154.

[7] C. Chen, D. Gal'tsov, and S. Sharakin, "Intersecting M-fluxbranes," Grav. Cosmol. 5 (1999) 45-48. arXiv:hep-th/9908132.

[8] M. Costa and M. Gutperle, "The Kaluza-Klein Melvin solution in M-theory," J. High Energy Phys. 03 (2001) 027. arXiv:hep-th/0012072.

[9] M. Gutperle and A. Strominger, "Fluxbranes in string theory," $J$. High Energy Phys. 06 (2001) 035. arXiv:hep-th/0104136.

[10] P. Saffin, "Gravitating fluxbranes," Phys. Rev. D64 (2001) 024014. arXiv: gr-qc/0104014.

[11] M. Costa, C. Herdeiro, and L. Cornalba, "Fluxbranes and the dielectric effect in string theory." arXiv:hep-th/0105023.

[12] R. Emparan, "Tubular branes in fluxbranes," Nucl. Phys. B610 (2001) 169-189. arXiv:hep-th/0105062.

[13] D. Brecher and P. Saffin, "A note on the supergravity description of dielectric branes," Nucl. Phys. B613 (2001) 218-236. arXiv: hep-th/0106206.

[14] A. Uranga, "Wrapped fluxbranes." arXiv:hep-th/0108196.

[15] R. Emparan and M. Gutperle, "From $p$-branes to fluxbranes and back," J. High Energy Phys. 12 (2001) 023. arXiv:hep-th/0111177. 
[16] J. Figueroa-O'Farrill and J. Simón, "Generalised supersymmetric fluxbranes," J. High Energy Phys. 12 (2001) 011. arXiv: hep-th/0110170.

[17] H. Liu, G. Moore, and N. Seiberg, "Strings in time-dependent orbifolds." arXiv:hep-th/0206182.

[18] J. Russo and A. Tseytlin, "Green-Schwarz superstring action in a curved magnetic Ramond-Ramond background," J. High Energy Phys. 04 (1998) 014. arXiv:hep-th/9804076.

[19] J. Russo and A. Tseytlin, "Magnetic backgrounds and tachyonic instabilities in closed superstring theory and M-theory," Nucl. Phys. B611 (2001) 93-124. arXiv: hep-th/0104238.

[20] A. Tseytlin, "Magnetic backgrounds and tachyonic instabilities in closed string theory." arXiv:hep-th/0108140.

[21] T. Suyama, "Properties of string theory on Kaluza-Klein Melvin background." arXiv:hep-th/0110077.

[22] T. Takayanagi and T. Uesugi, "Orbifolds as Melvin geometry," J. High Energy Phys. 12 (2001) 004. arXiv:hep-th/0110099.

[23] J. Russo and A. Tseytlin, "Supersymmetric fluxbrane intersections and closed string tachyons." arXiv:hep-th/0110107.

[24] G. Horowitz and A. Steif, "Singular string solutions with nonsingular initial data," Phys. Lett. B258 (1991) 91.

[25] A. Tseytlin, "Exact string solutions and duality." arXiv:hep-th/9407099; C Klimcik and AA Tseytlin, unpublished (1994); AA Tseytlin, unpublished (2001).

[26] J. Simón, "The geometry of null rotation identifications," J. High Energy Phys. 06 (2002) 001.

[27] H. Liu, G. Moore, and N. Seiberg, "Strings in a time-dependent orbifold," J. High Energy Phys. 06 (2002) 045. arXiv:hep-th/0204168.

[28] G. Horowitz and J. Polchinski, "Instability of spacelike and null orbifold singularities." arXiv: hep-th/0206228.

[29] M. Fabinger and J. McGreevy, "On smooth time-dependent orbifolds and null singularities." arXiv:hep-th/0206196. 
[30] J. Figueroa-O'Farrill, "Breaking the M-waves," Class. Quant. Grav. 17 (2000) 2925-2947. arXiv:hep-th/9904124.

[31] R. Bryant, "Pseudo-Riemannian metrics with parallel spinor fields and vanishing Ricci tensor." arXiv:math.DG/0004073.

[32] M. Gutperle and A. Strominger, "Spacelike branes," J. High Energy Phys. 04 (2002) 018. arXiv:hep-th/0202210.

[33] C. Chen, D. Gal'tsov, and M. Gutperle, "S-brane solutions in supergravity theories," Phys. Rev. D66 (2002) 024043.

arXiv: hep-th/0204071.

[34] M. Kruczenski, R. Myers, and A. Peet, "Supergravity S-branes," J. High Energy Phys. 05 (2002) 039. arXiv:hep-th/0204144.

[35] N. Deger and A. Kaya, "Intersecting S-brane solutions of $D=11$ supergravity," J. High Energy Phys. 07 (2002) 038. arXiv:hep-th/0206057.

[36] A. Sen, "Rolling tachyon," J. High Energy Phys. 04 (2002) 048. arXiv: hep-th/0203211.

[37] A. Sen, "Time evolution in open string theory." arXiv:hep-th/0207105.

[38] O. Aharony, M. Fabinger, G. Horowitz, and E. Silverstein, "Clean time-dependent string backgrounds from bubble baths," J. High Energy Phys. 07 (2002) 007. arXiv:hep-th/0204158.

[39] V. Balasubramanian and S. Ross, "The dual of nothing." arXiv:hep-th/0205290.

[40] R. Cai, "Constant curvature black hole and dual field theory." arXiv: hep-th/0206223.

[41] A. Ghezelbash and R. Mann, "Nutty bubbles." arXiv: hep-th/0207123.

[42] A. Buchel, P. Langfelder, and J. Walcher, "On time-dependent backgrounds in supergravity and string theory." arXiv: hep-th/0207214.

[43] S. Elitzur, A. Giveon, D. Kutasov, and E. Rabinovici, "From big bang to big crunch and beyond." arXiv:hep-th/0204189, 2002.

[44] B. Craps, D. Kutasov, and G. Rajesh, "String propagation in the presence of cosmological singularities." arXiv: hep-th/0205101, 2002. 
[45] J. Khoury, B. Ovrut, N. Seiberg, P. Steinhardt, and N. Turok, "From big crunch to big bang," Phys. Rev. D65 (2002) 086007. arXiv:hep-th/0108187.

[46] N. Seiberg, "From big crunch to big bang - is it possible?." arXiv: hep-th/0201039.

[47] V. Balasubramanian, S. Hassan, E. Keski-Vakkuri, and A. Naqvi, "A space-time orbifold: a toy model for a cosmological singularity." arXiv:hep-th/0202187.

[48] L. Cornalba and M. Costa, "A new cosmological scenario in string theory." arXiv:hep-th/0203031.

[49] N. Nekrasov, "Milne universe, tachyons, and quantum group." arXiv:hep-th/0203112.

[50] T. Takayanagi and T. Uesugi, "D-branes in Melvin background," $J$. High Energy Phys. 11 (2001) 036. arXiv: hep-th/0110200.

[51] E. Dudas and J. Mourad, "D-branes in string theory Melvin backgrounds," Nucl. Phys. B622 (2002) 46-72. arXiv:hep-th/0110186.

[52] J. Simón, "D0-branes in flux 5-brane backgrounds," Nucl. Phys. B635 (2002) 175-191. arXiv:hep-th/0201061.

[53] J. Figueroa-O'Farrill and J. Simón, "Supersymmetric Kaluza-Klein reductions of M-waves and MKK-monopoles." arXiv: hep-th/0208108.

[54] J. Figueroa-O'Farrill, H. Rajaniemi, and J. Simón, "Supersymmetric Kaluza-Klein reductions of intersecting M-branes." in preparation.

[55] O. Aharony, S. Gubser, J. Maldacena, H. Ooguri, and Y. Oz, "Large N field theories, string theory and gravity," Phys. Rep. 323 (2000) 183-386. arXiv:hep-th/9905111.

[56] A. Bergman and O. Ganor, "Dipoles, twists and noncommutative gauge theory," J. High Energy Phys. 10 (2000) 018. arXiv: hep-th/0008030.

[57] K. Dasgupta, O. Ganor, and G. Rajesh, "Vector deformations of $N=4$ super-Yang-Mills theory, pinned branes, and arched strings," J. High Energy Phys. 04 (2001) 034. arXiv:hep-th/0010072. 
[58] A. Bergman, K. Dasgupta, O. Ganor, J. Karczmarek, and G. Rajesh, "Nonlocal field theories and their gravity duals," Phys. Rev. D65 (2002) 066005. arXiv:hep-th/0103090.

[59] K. Dasgupta and M. Sheikh-Jabbari, "Noncommutative dipole field theories," J. High Energy Phys. 02 (2002) 002.

arXiv:hep-th/0112064.

[60] M. Alishahiha and H. Yavartanoo, "Supergravity description of the large N noncommutative dipole field theories," J. High Energy Phys. 04 (2002) 031. arXiv:hep-th/0202131.

[61] J. Maldacena, "The large $N$ limit of superconformal field theories and supergravity," Adv. Theor. Math. Phys. 2 (1998) 231-252.

arXiv:hep-th/9711200.

[62] N. Itzhaki, J. Maldacena, J. Sonnenschein, and S. Yankielowicz, "Supergravity and the large $N$ limit of theories with sixteen supercharges." arXiv:hep-th/9802042.

[63] L. Motl, "Melvin matrix models." arXiv:hep-th/0107002.

[64] J. Morales and M. Trigiante, "Walls from fluxes: An analytic RG-flow," J. High Energy Phys. 02 (2002) 018. arXiv: hep-th/0112059.

[65] M. Bañados, C. Teitelboim, and J. Zanelli, "The black hole in three-dimensional spacetime," Phys. Rev. Lett. 69 (1992) 1849.

[66] M. Bañados, M. Henneaux, C. Teitelboim, and J. Zanelli, "Geometry of the $2+1$ black hole," Phys. Rev. D48 (1993) 1506.

arXiv: gr-qc:9302012.

[67] J. Simón, "Null orbifolds in AdS, time dependence and holography." arXiv: hep-th/0208165.

[68] J. Figueroa-O'Farrill and J. Simón, "Supersymmetric Kaluza-Klein reductions of AdS backgrounds." in preparation.

[69] W. Nahm, "Supersymmetries and their representations," Nucl. Phys. B135 (1978) 149-166.

[70] E. Cremmer, B. Julia, and J. Scherk, "Supergravity in eleven dimensions," Phys. Lett. 76B (1978) 409-412.

[71] I. Campbell and P. West, " $N=2 D=10$ nonchiral supergravity and its spontaneous compactification," Nucl. Phys. B243 (1984) 112. 
[72] F. Giani and M. Pernici, " $N=2$ supergravity in ten dimensions," Phys. Rev. D30 (1984) 325-333.

[73] M. Huq and M. Namazie, "Kaluza-Klein supergravity in ten dimensions," Class. Quant. Grav. 2 (1985) 293.

[74] Y. Kosmann, "Dérivées de Lie des spineurs," Annali di Mat. Pura Appl. (IV) 91 (1972) 317-395.

[75] J. Figueroa-O'Farrill, "On the supersymmetries of Anti-de Sitter vacua," Class. Quant. Grav. 16 (1999) 2043-2055. arXiv: hep-th/9902066.

[76] M. Duff and K. Stelle, "Multi-membrane solutions of $D=11$ supergravity," Phys. Lett. 253B (1991) 113-118.

[77] E. Cremmer, B. Julia, H. Lu, and C. Pope, "Dualisation of dualities. I," Nucl. Phys. B523 (1998) 73-144. arXiv:hep-th/9710119.

[78] E. Bergshoeff, C. Hull, and T. Ortín, "Duality in the type-II superstring effective action," Nucl. Phys. B451 (1995) 547-578. arXiv: hep-th/9504081.

[79] C. Hull, "Time-like T-duality, de Sitter space, large $N$ gauge theories, and topological field theory," J. High Energy Phys. 07 (1998) 021. arXiv:hep-th/9806146.

[80] C. Hull, "Duality and the signature of space-time," J. High Energy Phys. 11 (1998) 017. arXiv:hep-th/9807127.

[81] C. Hull and R. Khuri, "Branes, times and dualities," Nucl. Phys. B536 (1998) 219-244. arXiv:hep-th/9808069.

[82] R. Güven, "Black $p$-brane solutions of $D=11$ supergravity theory," Phys. Lett. 276B (1992) 49-55.

[83] O. Aharony, M. Berkooz, D. Kutasov, and N. Seiberg, "Linear dilatons, NS5-branes and holography," J. High Energy Phys. 10 (1998) 004. arXiv:hep-th/9808149.

[84] G. Horowitz and A. Strominger, "Black strings and p-branes," Nucl. Phys. B360 (1991) 197-209. 